Pure and Applied Mathematics Quarterly

Volume 2, Number 1

(Special Issue: In honor of

John H. Coates, Part 1 of 2$)$

$219-277,2006$

\title{
Periods and Distribution of Cycles on Hilbert Modular Varieties
}

\author{
Di-Hua Jiang, Jian-Shu Li and Shou-Wu Zhang \\ To Professor John Coates, with admiration
}

\section{Contents}

1. Introduction 220

1.1. Hilbert modular varieties 220

1.2. Main Theorems 221

Acknowledgement 224

2. Equidistributions 224

2.1. Geometric setting 225

2.2. On Hecke orbits 228

2.3. From Theorem 1.2.1 to Theorem 1.2.3 231

3. Periods of Eisenstein series 233

3.1. First computations 233

3.2. Godement-Jacquet integrals 236

3.3. Volumes of Shimura subvarieties 240

4. Theta lifting 242

4.1. The Isogeny 243

4.2. Theta lifting 245

4.3. The Siegel-Weil formula 253

Received April 13, 2005. 
4.4. Theta Lifts for Eisenstein Series

5. Periods of cusp forms 259

5.1. Split case 259

5.2. Gelbart-Jacquet integrals 263

5.3. On normalization of theta lifting 268

$\begin{array}{ll}\text { 5.4. On ratios } & 274\end{array}$

$\begin{array}{ll}\text { References } & 276\end{array}$

\section{INTRODUCTION}

The objective of this paper is to prove an explicit identity between period integrals of automorphic forms and special values of automorphic L-series. As an application, we will show that certain Shimura subvarieties on Hilbert modular varieties are equidistributed. In the following we describe the main results.

1.1. Hilbert modular varieties. Let $F$ be a totally real field with degree $d$. Let $\mathbf{G}=\operatorname{Res}_{F / \mathbb{Q}} \mathrm{PGL}_{2}$. Then the group $\mathbf{G}(\mathbb{R})=\mathrm{PGL}_{2}(\mathbb{R})^{d}$ acts on the product $X:=\left(\mathcal{H}^{ \pm}\right)^{d}$ of Poincaré double-half planes. Let $\mathbb{A}$ be the ring of adeles of $\mathbb{Q}$ and write $\mathbb{A}=\mathbb{R} \times \widehat{\mathbb{Q}}$, where $\widehat{\mathbb{Q}}=\mathbb{A}_{f}$ is the finite adeles of $\mathbb{Q}$. For each open and compact subgroup $U$ of $\mathbf{G}(\widehat{\mathbb{Q}})$ we have a Hilbert modular variety $\mathcal{M}_{U, \mathbb{C}}$ over $\mathbb{C}$ whose complex points are given by

$$
\mathcal{M}_{U, \mathbb{C}}(\mathbb{C}):=\mathbf{G}(\mathbb{Q}) \backslash X \times \mathbf{G}(\widehat{\mathbb{Q}}) / U .
$$

As $U$ varies the family $\left\{\mathcal{M}_{U, \mathbb{C}}\right\}$ forms a projective system of quasi-projective varieties over $\mathbb{C}$. This system has a natural action by $\mathbf{G}(\widehat{\mathbb{Q}})$. Let $F_{+}$denote the group of totally positive elements in $F$. The set of geometric connected components over $\mathbb{C}$ is given by

$$
\pi_{0}\left(\mathcal{M}_{U, \mathbb{C}}\right) \simeq F^{\times} \backslash\{ \pm 1\}^{d} \times \widehat{F}^{\times} / \operatorname{det}(U)=F_{+}^{\times} \backslash \widehat{F}^{\times} / \operatorname{det}(U)
$$

with the map $\mathcal{M}_{U, \mathbb{C}}(\mathbb{C}) \longrightarrow \pi_{0}\left(\mathcal{M}_{U, \mathbb{C}}(\mathbb{C})\right)$ given by taking determinants.

The homomorphism from $\mathbb{C}^{\times}$to $\mathrm{GL}_{2}(\mathbb{R})^{d}$ which sends $x+y i$ to

$$
\left(\left(\begin{array}{cc}
x & y \\
-y & x
\end{array}\right), \cdots,\left(\begin{array}{cc}
x & y \\
-y & x
\end{array}\right)\right)
$$

defines a morphism $h_{0}$ from $\mathbb{S}=\operatorname{Res}_{\mathbb{C} / \mathbb{R}}\left(\mathrm{GL}_{1}\right)$ to $\mathbf{G}_{\mathbb{R}}$. The space $X$ can be identified with the $\mathbf{G}(\mathbb{R})$-conjugacy class of $h_{0}$. With respect to $h_{0}$, the system of varieties $\mathcal{M}_{U, \mathbb{C}}$ have canonical models $\mathcal{M}_{U}$ over $\mathbb{Q}$ which is compatible with the action of $\mathbf{G}(\widehat{\mathbb{Q}})$. Thus the variety $\mathcal{M}_{U, \mathbb{C}}$ has a (left) action of $\operatorname{Aut}(\mathbb{C})$ which 
commutes with the right action of $\mathbf{G}(\widehat{\mathbb{Q}})$. Every geometric component of $\mathcal{M}_{U, \mathbb{C}}$ is defined over $\mathbb{Q}^{\mathrm{ab}}$. The induced action of $\operatorname{Gal}(\overline{\mathbb{Q}} / \mathbb{Q})$ on $\pi_{0}\left(\mathcal{M}_{U}\right)$ is given by the composition of the class field theory map

$$
\operatorname{Gal}\left(\mathbb{Q}^{\mathrm{ab}} / \mathbb{Q}\right) \simeq \mathbb{Q}_{+}^{\times} \backslash \widehat{\mathbb{Q}}^{x}
$$

and the multiplication of $\widehat{\mathbb{Q}}^{\times}$on $\widehat{F}^{\times}$. Here the class field theory map is normalized such that the geometric Frobenius corresponds to the uniformizers. We refer to Deligne's paper [5] for the definition of canonical models but notice that our Galois action is inverse to (the right) Galois action used in Deligne's paper.

Let us recall the description of Shimura subvarieties of $\mathcal{M}_{U}$. For each point $x \in \mathcal{M}_{U}(\mathbb{C})$, the minimal Shimura subvariety of Hodge type containing $x$ can be defined as follows. Let $\left(h, \tau_{f}\right) \in X \times \mathrm{GL}_{2}(\widehat{\mathbb{Q}})$ represent $x$. Let $\mathbf{H}$ denote the Zariski closure of $h(\mathbb{S})$ in $\mathbf{G}$ as an algebraic subgroup over $\mathbb{Q}$ which is called the Mumford-Tate group of $x$. The Hodge closure $\mathcal{M}_{U, x}$ of $x$ in $\mathcal{M}_{U}$ is defined to be the subvariety of $\mathcal{M}$ represented by $\mathbf{H}(\mathbb{R}) h \times \mathbf{H}(\widehat{\mathbb{Q}}) \tau_{f}$. The minimal Shimura subvariety $\mathcal{M}_{U, x}$ of Hodge type containing $x$ has the form

$$
\mathcal{M}_{U, x}:=\mathbf{H}(\mathbb{Q}) \backslash X^{\prime} \times\left(\mathbf{H}(\widehat{\mathbb{Q}}) / U^{\prime}\right) \tau_{f}, \quad U^{\prime}:=\mathbf{H}(\widehat{\mathbb{Q}}) \cap \tau_{f} U \tau_{f}^{-1}, \quad X^{\prime}:=\mathbf{H}(\mathbb{R}) h .
$$

There are basically two different types of Shimura subvarieties for $\mathcal{M}_{U}$ up to conjugations:

- Let $k$ be a subfield of $F$ and $D$ be a quaternion algebra over $k$ with a fixed embedding into $M_{2}(F)$. Take $\mathbf{H}$ to be the group $\operatorname{Res}_{k / \mathbb{Q}}\left(D^{\times} / k^{\times}\right)$. The variety so obtained is called a quaternion subvariety. All these varieties are defined over $\mathbb{Q}$ with Galois action given by the reciprocity law and the homomorphism

$$
\mathrm{GL}_{1} \longrightarrow \operatorname{Res}_{k / \mathbb{Q}} \mathrm{GL}_{1}
$$

- Let $K$ be a totally imaginary quadratic extension of $F$ embedded in $M_{2}(F)$. Take $\mathbf{H}$ to be the group $K^{\times}$. The variety so obtained is a set of points, which are called CM-points on $\mathcal{M}_{U}$. All CM-points are defined over abelian extensions of a certain reflex field $\widetilde{K}$.

1.2. Main Theorems. Let $k$ be a fixed subfield of $F$ such that $[F: k]=2$. Let $\mathcal{M}:=\mathcal{M}_{U}$ be the Hilbert modular variety with $U$ the standard maximal compact subgroup $\mathrm{PGL}_{2}\left(\widehat{\mathcal{O}}_{F}\right)$. Let $\mathcal{M}^{0}$ be the unit connected component of $\mathcal{M}_{U}$ corresponding to the unit element in $\pi_{0}(\mathcal{M})=F_{+}^{\times} \backslash \widehat{F}^{\times} / \operatorname{det} U$. Let $x \in$ $\mathcal{M}^{0}$ be a point such that the Mumford-Tate group $\mathbf{H}$ of $x$ has the form $\mathbf{H}=$ $\operatorname{Res}_{k / \mathbb{Q}}\left(D^{\times} / k^{\times}\right)$for some quaternion algebra over $k$ embedded into $M_{2}(F)$.

Let $\pi$ be an irreducible automorphic representation of $\mathrm{PGL}_{2}\left(\mathbb{A}_{F}\right)$ which is spherical with weight 0 . Let $\varphi \in \pi$ be its newform. By definition, $\varphi$ is the unique 
spherical function in $\pi$ such that

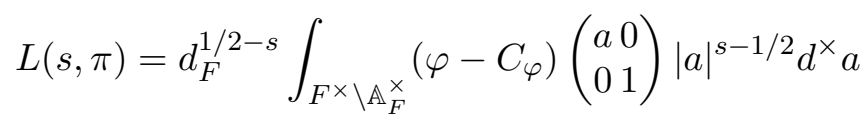

where $d_{F}$ is the absolute discriminant of $F, C_{\varphi}$ is the constant term of $\varphi$, and the measure $d^{\times} a=\prod_{v} d^{\times} a_{v}$ is the product of local measures $d^{\times} a_{v}$ such that $\operatorname{vol}\left(\mathcal{O}_{v}^{\times}\right)=1$ if $v$ is finite and $d^{\times} a_{v}=d a_{v} / a_{v}$ if $v$ is infinite. We will try to get a formula for the integration of $\varphi$ on $\mathcal{M}_{x}$ with respect to an invariant measure $d h$ on $\mathcal{M}_{x}$ :

$$
\ell_{x}(\varphi)=\operatorname{vol}\left(\mathcal{M}_{x}(\mathbb{C})\right)^{-1} \int_{\mathcal{M}_{x}(\mathbb{C})} \varphi(h) d h .
$$

Theorem 1.2.1. Assume that either $\mathcal{M}_{x}$ is compact or $\varphi$ is cuspidal, that the Hodge closure $\mathcal{M}_{x}$ has minimal level, and that the integral $\ell_{x}(\varphi) \neq 0$. Then $\pi$ is a base change of an automorphic representation $\sigma$ of $\mathrm{GL}_{2}\left(\mathbb{A}_{k}\right)$ with central character $\eta$ associated to the extension $F / k$. Moreover the value of the integral is given by the following formula:

$$
\ell_{x}(\varphi)=\frac{1}{\sqrt{d_{k}} \zeta_{k}(2)} \cdot \prod_{v \mid \delta_{D}} \frac{\chi_{v}\left(\varpi_{v}\right)-\chi_{v}\left(\varpi_{v}\right)^{-1}}{q_{v}^{1 / 2}-q_{v}^{-1 / 2}} \cdot L\left(1, \operatorname{Sym}^{2} \sigma\right) .
$$

Here

- $d_{k}$ is the absolute discriminant of $k$;

- $\delta_{D}$ is the discriminant ideal of $\mathcal{O}_{k}$ for $D$;

- $q_{v}$ is the cardinality of the residue field of $\mathcal{O}_{k, v}$ at $v$;

- where $\chi_{v}$ is an unramified character of $k_{v}^{\times}$such that $\sigma_{v} \simeq \pi\left(\chi_{v}, \chi_{v}^{-1} \eta_{v}\right), \eta_{v}$ is the $v$-component of the central character $\eta$, and $\varpi_{v}$ is a local parameter of $k_{v}$.

Remark 1.2.2. 1. The proof of Theorem can be extended to more general cases: where $\mathcal{M}$ may have some level $N$, $\phi$ may be a form for the representation $\Pi$ with central character of the form $\chi \circ \mathrm{N}_{F / k}$ with conductor $c(\omega)$ prime to $N$, and $M_{x}$ is the quaternion algebra with discriminant prime to $c(\omega) N$. We will have the periods

$$
\ell_{\mathbf{H}}(\varphi):=\int_{\mathbf{H}(\mathbb{Q}) \backslash \mathbf{H}(\mathbb{A})} \varphi(h) \chi^{-1}(\operatorname{det} h) d h .
$$

The nonvanishing of this period will imply that $\pi$ is the base change of a representation $\sigma$ of $\mathrm{GL}_{2}\left(\mathbb{A}_{k}\right)$ with central character $\chi \cdot \eta$. The period $\ell_{\mathbf{H}}(\varphi)$ is equal to a constant times the value at $s=1$ of a certain twisted adjoint $L$-function of $\sigma$.

2. The equivalence between the nonvanishing of period integral and the base change is well known, which was first proved by Harder, Langlands, and Rapoport in their work [8] on the Tate's conjecture at least when $D$ is split and $\pi$ is holomorphic of parallel weight 2. Our explicit formula has two applications: one is the 
equidistribution theorem described as below, and another is about the refinement of the Tate's conjecture given by Bloch and Kato [2] which will be considered in our future work.

In the following we want to describe an application to the distribution of Shimura subvarieties with arbitrary level. With notations as above, let $\mathcal{M}_{x}^{0}$ denote the intersection between $\mathcal{M}^{0}$ and $\mathcal{M}_{x}$. Now let $\varphi$ be a function on $\mathcal{M}^{0}(\mathbb{C})$ which is smooth with compact support. We want to compute the integral

$$
\ell_{x}^{0}(\varphi):=\operatorname{vol}\left(\mathcal{M}_{x}^{0}(\mathbb{C})\right)^{-1} \int_{\mathcal{M}_{x}^{0}(\mathbb{C})} \varphi(h) d h
$$

with respect to an invariant measure on $\mathcal{M}_{x}^{0}(\mathbb{C})$. To state our result, we define the order $\mathcal{O}_{x} \subset D(k)$ by the formula

$$
\mathcal{O}_{x}=D(k) \cap \tau_{f} M_{2}\left(\widehat{\mathcal{O}}_{F}\right) \tau_{f}^{-1}
$$

where $\tau_{f}$ is determined by $x$ by definition. Let $\delta_{x}$ denote the (reduced) discriminant ideal of this order $\mathcal{O}_{k}$ with respect to canonical pairing $D(k) \times D(k) \longrightarrow k$ given by

$$
(x, y) \mapsto \operatorname{tr}_{D / k}(x y) .
$$

We will show that there are only finitely many quaternion Shimura subvarieties $\mathcal{M}_{x}$ with bounded discriminants $\mathrm{N}_{k / \mathbb{Q}} \delta_{x}$.

We also need a bound $\alpha$ for the Fourier coefficients of Maass forms $f$. Write the local L-factor of the standard L-function $L(s, f)$ as

$$
L_{v}(s, f)=\frac{1}{\left(1-a_{v}(f)\left|\omega_{v}\right|_{v}^{s}\right)\left(1-a_{v}^{-1}(f)\left|\omega_{v}\right|_{v}^{s}\right)} .
$$

Then $\alpha$ is a positive number such that there are constant $C$ and $A$ depending only on field $k$ such that

$$
\max \left(\left|a_{v}(f)\right|,\left|a_{v}(f)\right|^{-1}\right) \leq C \lambda(f)^{A} q_{v}^{\alpha}
$$

for every Maass eigen form $f$ for $\mathrm{PGL}_{2}\left(\mathbb{A}_{k}\right)$, where $\lambda(f)$ is the eigenvalues of $f$ under the Laplace operator, and $q_{v}$ is the cardinality of the residue field of $\mathcal{O}_{k}$. The Peterson-Ramanujan conjecture says that the absolute value of $a_{v}(f)$ is always 1 . So $\alpha$ could be any positive number. The recent work of Kim-Shahidi [13] implies that the inequality hold for $\alpha=1 / 9+\epsilon$.

Theorem 1.2.3. For any $\epsilon>0$ there is a positive number $C$ such that

$$
\left|\ell_{x}^{0}(\varphi)\right| \leq C \cdot \mathrm{N}_{k / \mathbb{Q}}\left(\delta_{x}\right)^{\varepsilon+\alpha-1 / 2} .
$$

Thus we have the following equidistribution statement with precise rate of convergence for quaternion Shimura varieties (with same or different quaternion algebras): 
Corollary 1.2.4. Let $\mathcal{M}_{i}$ be an infinite sequence of distinct compact quaternion Shimura subvarieties of $\mathcal{M}$ defined by some $x$ as above. Let $\varphi$ be a smooth function on $\mathcal{M}^{0}(\mathbb{C})$ with compact support. Then $\ell_{\mathcal{M}_{i}^{0}}(\varphi)$ converges to $\int_{\mathcal{M}^{0}(\mathbb{C})} \varphi$ as $i \longrightarrow \infty$.

The above corollary is a very special case of the following conjecture:

Conjecture 1.2.5. Let $\mathcal{M}_{i}$ be a sequence Shimura subvarieties of $\mathcal{M}_{U}$ defined over a fixed number field $E$. Assume that no subsequence of $\mathcal{M}_{i}$ is included in a proper Shimura subvariety of $\mathcal{M}_{U}$. Then $\mathcal{M}_{i}$ is equidistributed.

Remark 1.2.6. 1. A general statement for the so called strongly special subvarieties $\mathcal{M}_{i}$ defined by semi-simple group $\mathbf{H}$ has been proved by Clozel-Ullmo [3] using ergodic theory. This includes the case of our Corollary 1.2.4 without precise rate of convergence.

2. It is not difficult to see that Conjecture 1.2.5 for zero-dimensional subvarieties $\mathcal{M}_{i}$ implies the André-Oort conjecture, which is stated below.

Conjecture 1.2.7 (André-Oort, [1], [16], [15]). Let $S$ be a set of CM-points on $\mathcal{M}_{U}$. Then the Zariski closure $\bar{S}$ of $S$ is a finite union of Shimura subvarieties of $\mathcal{M}_{U}$.

Under certain assumption on subconvexity bound for L-series, Conjecture 1.2.5 has been established for CM-points with the maximal Mumford-Tate group by one of the authors, see [21] for details.

Acknowledgement. The second named author was partially supported by RGCCERG grants HKUST6115/02P and HKUST601805. Jiang and Zhang would like to thank the Hong Kong University of Science and Technology for its hospitality during the course of this collaboration. Finally we wish to thank the Chinese Academy of Science and the US National Science Foundation for their continued generous support.

\section{Equidistributions}

In this section we will show that the estimate of our periods (Theorem 1.2.3) is a consequence of the explicit formula of the periods (Theorem 1.2.1). We start with a classification of quaternion subvarieties in terms of conductors. Then we show that the cycles generated by these subvarieties are generated by minimal ones. Finally we show that the equidistribution property follows from the estimates of the Fourier coefficients of the forms, and special values of the L-series. Note that we re-introduce notations for the rest of this paper, which are close to, but may be different from those in $\S 1$. 
2.1. Geometric setting. Let $k$ be a fixed subfield of $F$ such that $[F: k]=2$ and $D$ be a fixed quaternion algebra over $k$. Let $\iota: D(k) \longrightarrow M_{2}(F)$ be an embedding of $k$-algebras. Note that the existence of such $\iota$ is equivalent to $D$ being split over $F$. Let $\mathbf{G}=\operatorname{Res}_{F / \mathbb{Q}}\left(\mathrm{PGL}_{2}\right)$ be the restriction by scalar of $\mathrm{PGL}_{2}$, which defines an algebraic group defined over $\mathbb{Q}$. Let $\mathbf{H}=\operatorname{Res}_{k / \mathbb{Q}}\left(D^{\times} / Z_{D^{\times}}\right)$ be the restriction by scalar of $D^{\times} / Z_{D^{\times}}\left(Z_{D^{\times}}\right.$is the center of $\left.D^{\times}\right)$, which is an algebraic group defined over $\mathbb{Q}$ and is viewed as an algebraic subgroup of $\mathbf{G}$ via $\iota$.

Let $H=D^{\times} / Z_{D^{\times}}$, so that

$$
\mathbf{H}(\mathbb{Q})=H(k), \text { and } \quad \mathbf{G}(\mathbb{Q})=\mathrm{PGL}_{2}(F) .
$$

We denote by $\mathbb{A}$ the ring of adeles of $\mathbb{Q}$. Let $\tau \in \mathbf{G}(\mathbb{A})=\mathrm{PGL}_{2}\left(\mathbb{A}_{F}\right)$ be an element with the Mumford-Tate group $\mathbf{H}$. Then the right multiplication by $\tau$ gives rise to a morphism

$$
\iota_{\tau}: \quad \mathcal{M}_{D, \tau}:=\mathbf{H}(\mathbb{Q}) \backslash \mathbf{H}(\mathbb{A}) / U_{D, \tau} \longrightarrow \mathcal{M}=\mathbf{G}(\mathbb{Q}) \backslash \mathbf{G}(\mathbb{A}) / U
$$

where $U$ is the standard maximal compact subgroup of $\mathbf{G}(\mathbb{A})$ and $U_{D, \tau}=\tau U \tau^{-1} \cap$ $\mathbf{H}(\mathbb{A})$. Note that $\tau$ has Mumford-Tate group $\mathbf{H}$ if and only if for every infinite place $v$ of $k$,

- $\left(U_{D, \tau}\right)_{v}$ is a maximal connected compact subgroup of $H\left(k_{v}\right)$,

- the manifold $H\left(k_{v}\right) /\left(U_{D, \tau}\right)_{v}$ has a complex structure via the embedding $\iota_{\tau_{v}}$.

Our first task is to classify the subvarieties

$$
\mathcal{M}_{\iota, \tau}:=\iota_{\tau}\left(\mathcal{M}_{D, \tau}\right)
$$

of $\mathcal{M}$. For each $\tau$ with the Mumford-Tate group $\mathbf{H}$ as above, we define the order $\mathcal{O}_{\iota, \tau} \subset D(k)$ by the formula

$$
\iota\left(\mathcal{O}_{\iota, \tau}\right)=\iota(D(k)) \cap \tau M_{2}\left(\widehat{\mathcal{O}}_{F}\right) \tau^{-1} .
$$

Let $\delta_{\iota, \tau}$ denote the (reduced) discriminant ideal in $\mathcal{O}_{k}$ of this order with respect to the canonical pairing $D(k) \times D(k) \longrightarrow k$ given by

$$
(x, y) \mapsto \operatorname{tr}_{D / k}(x y) .
$$

Proposition 2.1.1. Fix a quaternion algebra $D$ over $k$ which is split over $F$. The map

$$
\mathcal{M}_{\iota, \tau} \longrightarrow \delta_{\iota, \tau}
$$

is well defined and bijective between the set of Shimura subvarieties of $\mathcal{M}$ defined by some data $(\iota, \tau)$ and the set of nonzero ideals of $\mathcal{O}_{k}$ of the type $\delta_{D} \cdot \mathrm{N}_{F / k} \mathcal{N}$ where $\delta_{D}$ is the discriminant of $D$ and $\mathcal{N}$ is an ideal in $\mathcal{O}_{F}$. 
We call $c=\mathrm{N}_{F / k} \mathcal{N}$ the conductor of $\mathcal{M}_{c}:=\mathcal{M}_{\iota, \tau}$.

The proof of Proposition 2.1.1 reduces to those of Lemma 2.1.2 and Lemma 2.1.3. First, we show the map given in Proposition 2.1.1 is well-defined.

Since $D$ is split over $F$, via the embedding $\iota$, we may write $D=F+F \lambda$ with $\lambda x=\bar{x} \lambda$ for all $x \in F$. Then the embedding $\iota$ can be expressed as

$$
x+y \lambda \mapsto \gamma\left(\begin{array}{l}
x y \epsilon \\
\bar{y} \\
\bar{x}
\end{array}\right) \gamma^{-1} .
$$

where $\gamma \in \mathrm{GL}_{2}(F)$ and $\epsilon=\lambda^{2} \in k^{\times}$. We write $\mathbf{H}_{\epsilon, \gamma}$ for the image of $\mathbf{H}$. Then

$$
\mathcal{M}_{\iota, \tau}=\mathbf{G}(\mathbb{Q}) \backslash \mathbf{G}(\mathbb{Q}) \mathbf{H}_{\epsilon, \gamma}(\mathbb{A}) \tau U / U=\mathbf{G}(\mathbb{Q}) \backslash \mathbf{G}(\mathbb{Q}) \mathbf{H}_{\epsilon, 1} \gamma^{-1} \tau U / U .
$$

With $\tau$ replaced by $\gamma^{-1} \tau$, we may assume $\gamma=1$ for all $\mathcal{M}_{\iota, \tau}$. The subvariety $\mathcal{M}_{\iota, \tau}$ is completely determined by the double coset of $\tau$ in $\mathbf{H}(\mathbb{A}) \backslash \mathbf{G}(\mathbb{A}) / U$.

It follows that for different choices of representatives in the double coset of $\tau$ the completion $\widehat{\mathcal{O}}_{\iota, \tau}$ of $\mathcal{O}_{\iota, \tau}$ in $D\left(\mathbb{A}_{k, f}\right)$ is conjugate to each other. Thus $\delta_{\iota, \tau}$ depends only on the double coset of $\tau$. Moreover the order $\mathcal{O}_{\iota, \tau}$ containing $\mathcal{O}_{F}$ will have the form

$$
\mathcal{O}_{F}+\mathcal{N} \mathcal{O}_{D}
$$

where $\mathcal{O}_{D}$ is a fixed maximal order of $D$ containing $\mathcal{O}_{F}$ and $\mathcal{N}$ is an ideal of $\mathcal{O}_{F}$. The discriminant of this order is given by $\delta_{D} \cdot \mathrm{N}_{F / k} \mathcal{N}$. Since the conjugacy class of $\widehat{\mathcal{O}}_{\iota, \tau}$ in $D\left(\mathbb{A}_{k, f}\right)$ depends only on $\delta_{\iota, \tau}$, the map in Proposition 2.1.1 is well-defined.

Now let us analyze the nature of $\tau_{\infty}$. By choosing different $\lambda$ in the decomposition, we may assume that

- $\epsilon$ is totally negative.

Lemma 2.1.2. The element $\tau$ has Mumford-Tate group $\mathbf{H}$ if and only if for every pair $\left(v_{1}, v_{2}\right)$ of conjugate real places of $F$ over a real place $v$ of $k$,

$$
\tau_{v_{2}} \in \lambda_{v} \tau_{v_{1}} \cdot \mathrm{SO}_{2}\left(k_{v}\right) \cdot k_{v}^{\times}
$$

where $\lambda_{v}=\left(\begin{array}{ll}0 & \epsilon \\ 1 & 0\end{array}\right)$

Proof. Fix a real place $v$ of $k$. The subgroup $H\left(k_{v}\right)$ of

$$
\mathrm{PGL}_{2}\left(F_{v}\right)=\mathrm{PGL}_{2}\left(k_{v}\right) \times \mathrm{PGL}_{2}\left(k_{v}\right)
$$

consists of pairs $\left(g_{1}, g_{2}\right)$ of the form

$$
g_{1}=\left(\begin{array}{ll}
a & b \epsilon \\
c & d
\end{array}\right), \quad g_{2}=\left(\begin{array}{ll}
d & c \epsilon \\
b & a
\end{array}\right) .
$$

In other words, $H\left(k_{v}\right)$ consists of pairs of type $\left(g, \lambda g \lambda^{-1}\right)$. Now, $\tau$ is of Hodge type if and only if the following two conditions are satisfied: 
- the subgroup $U_{v}$ of elements $g \in \mathrm{PGL}_{2}\left(k_{v}\right)$ such that

$$
\left(\tau_{v_{1}}^{-1} g \tau_{v_{1}}, \tau_{v_{2}}^{-1} \lambda_{v} g \lambda_{v}^{-1} \tau_{v_{2}}\right) \in \mathrm{SO}_{2}\left(k_{v}\right)^{2}
$$

is conjugate to $\mathrm{SO}_{2}\left(k_{v}\right)$.

- two maps

$$
\operatorname{Ad}\left(\tau_{v_{1}}\right), \operatorname{Ad}\left(\tau_{v_{2}} \lambda_{v}^{-1}\right): \quad \mathrm{GL}_{2}\left(F_{v}\right) / U_{v} \longrightarrow \mathcal{H}^{ \pm}
$$

induces the same complex structure on $\mathrm{GL}_{2}\left(F_{v}\right) / U_{v}$.

The first condition is equivalent to that

$$
\lambda_{v} \tau_{v_{1}} \mathrm{SO}_{2}\left(k_{v}\right) \tau_{v_{1}}^{-1} \lambda_{v}^{-1}=\tau_{v_{2}} \mathrm{SO}_{2}\left(k_{v}\right) \tau_{v_{2}}^{-1} .
$$

In other words,

$$
\tau_{v_{2}} \in \lambda_{v} \tau_{v_{1}} \cdot \mathrm{O}_{2}\left(k_{v}\right) \cdot k_{v}^{\times} .
$$

The second condition is equivalent to that $\operatorname{det}\left(\tau_{v_{1}}\right)$ has the same $\operatorname{sign} \operatorname{as} \operatorname{det}\left(\tau_{v_{2}} \lambda_{v}^{-1}\right)$. Thus we may replace $\mathrm{O}_{2}$ by $\mathrm{SO}_{2}$. The lemma follows.

This lemma shows that the class of $\tau_{\infty}$ in $\mathbf{H}(\mathbb{R}) \backslash \mathbf{G}(\mathbb{R}) / U_{\infty}$ is really fixed. We may simply fix such an element as

$$
\tau_{v}=\left(1, \lambda_{v}\right) . \quad(v \mid \infty)
$$

It remains to analyze the nature of $\tau_{f}$. Let $\mathcal{O}_{D}$ be any fixed maximal order of $D(k)$ containing $\mathcal{O}_{F}$. Let $\mathcal{N}$ be any ideal of $\mathcal{O}_{F}$ and consider the order

$$
\mathcal{O}_{\mathcal{N}}=\mathcal{O}_{F}+\mathcal{N} \mathcal{O}_{D}
$$

Let $\tau_{\mathcal{N}}$ be a fixed element in $\mathbf{G}\left(\mathbb{A}_{f}\right)$ such that

$$
\mathcal{O}_{F}^{2}=(0,1) \mathcal{O}_{\mathcal{N}} \tau_{\mathcal{N}}
$$

The existence of such $\tau_{\mathcal{N}}$ is obvious as $(0,1) \mathcal{O}_{\mathcal{N}}$ is an $\mathcal{O}_{F}$-lattice in $F^{2}$.

Lemma 2.1.3. The variety $\mathcal{M}_{\iota, \tau}$ has discriminant $\delta_{\iota, \tau}=\delta_{D} \cdot \mathrm{N}_{F / k}(\mathcal{N})$ if and only if

$$
\tau_{f} \in \mathbf{H}\left(\mathbb{A}_{f}\right) \cdot \tau_{\mathcal{N}} \cdot U
$$

Proof. Let $\Lambda$ be the lattice in $D$ defined by the equation

$$
\mathcal{O}_{F}^{2}=(0,1) \cdot \Lambda \cdot \tau_{f} .
$$

Then $\Lambda$ is a bi- $\mathcal{O}_{F}$ module. It is thus a left invertible module over some order $R$ of $D$ containing $\mathcal{O}_{F}$. In other words we have the equality

$$
\mathcal{O}_{F}^{2}=(0,1) R h \tau_{f}
$$

where $h \in \mathbf{H}\left(\mathbb{A}_{f}\right)$. Replacing $\tau$ by $h \tau$ we may assume that $h=1$. We claim that

$$
\tau_{f} M_{2}\left(\mathcal{O}_{F}\right) \tau_{f}^{-1} \cap D\left(\mathbb{A}_{k, f}\right)=R .
$$


Indeed for $g \in D\left(\mathbb{A}_{k, f}\right), g \in \tau_{f} M_{2}\left(\mathcal{O}_{F}\right) \tau_{f}^{-1}$ if and only if

$$
\mathcal{O}_{F}^{2} \tau_{f}^{-1} g \tau_{f} \subset \mathcal{O}_{F}^{2}
$$

This is equivalent to saying that $(0,1) g \tau_{f} \subset(0,1) R \tau_{f}$ or equivalently, that $g \in R$.

Now the discriminant $\delta_{\iota, \tau}$ equals $\delta_{D} \cdot \mathrm{N}_{F / k}(\mathcal{N})$. Thus $R$ is conjugate to $\mathcal{O}_{\mathcal{N}}$ by elements in $\mathbf{H}\left(\mathbb{A}_{f}\right)$. With $\tau$ replaced by a certain element in the coset $\mathbf{H}\left(\mathbb{A}_{f}\right) \tau_{f}$ we may simply assume that $R=\mathcal{O}_{\mathcal{N}}$. Now we have the equality

$$
\mathcal{O}_{F}^{2} \tau_{f}^{-1}=(0,1) \mathcal{O}_{N}=\mathcal{O}_{F}^{2} \tau_{\mathcal{N}}^{-1}
$$

Hence we must have $\tau_{f} \in \tau_{\mathcal{N}} U$.

2.2. On Hecke orbits. For the fixed quaternion algebra $D$ over $k$, which is embedded into $M_{2}(F)$ via $\iota$, we have just shown that all Shimura varieties $\mathcal{M}_{\iota, \tau}$ defined by certain elements $\tau$ of the Mumford-Tate group $D^{\times} / k^{\times}$are indexed by ideals $c$ of $\mathcal{O}_{k}$ which are norms from ideals $\mathcal{N}$ of $\mathcal{O}_{F}$. We may normalize $\mathcal{N}$ as follows: write $c=c^{\prime \prime}\left(c^{\prime}\right)^{2}$ with $c^{\prime \prime}$ square free, then $\mathcal{N}=c^{\prime} \mathcal{N}^{\prime \prime}$ with $c^{\prime \prime}=$ $\mathrm{N}_{F / k}\left(\mathcal{N}^{\prime \prime}\right)$.

The main result of this subsection is to show that the Shimura subvariety $\mathcal{M}_{c}:=\mathcal{M}_{\iota, \tau}$ of conductor $c$ is in the Hecke orbit of the Shimura subvariety $\mathcal{M}_{\text {min }}$ of minimal level. For an ideal $\mathfrak{n}$ of $\mathcal{O}_{F}$, let $T_{\mathfrak{n}}$ be the Hecke operator defined by integral matrices in $M_{2}(F)$ of determinant $n$. Now we define a Hecke operator $\mathrm{T}_{\mathfrak{n}}^{*}$ by the formula

$$
\mathrm{T}_{\mathfrak{n}}^{*}=\prod_{v} \mathrm{~T}_{\mathfrak{n}_{v}}^{*}
$$

where $\mathrm{T}_{\mathfrak{n}_{v}}^{*}$ is a Hecke operator at the place $v$ of $k$ given by

$$
\mathrm{T}_{\mathfrak{n}_{v}}^{*}=\mu\left(\mathfrak{n}_{v}\right)^{-1} \begin{cases}\mathrm{~T}_{\mathfrak{n}_{v}} & \text { if } v \mid \delta_{D} \\ \mathrm{~T}_{\mathfrak{n}_{v}}-\left(1+\left|\varpi_{v}\right|^{-1}\right) \mathrm{T}_{\mathfrak{n}_{v} / \varpi_{v}}+\left|\varpi_{v}\right|^{-1} \mathrm{~T}_{\mathfrak{n}_{v} / \varpi_{v}^{2}} & \text { otherwise. }\end{cases}
$$

where $\mu\left(\mathfrak{n}_{v}\right)$ is a number to make the degree of $T_{\mathfrak{n}_{v}}^{*}$ to be 1 . In other words,

$$
\mu\left(\mathfrak{n}_{v}\right)= \begin{cases}\sigma_{1}\left(\mathfrak{n}_{v}\right) & \text { if } v \mid \delta_{D} \\ \sigma_{1}\left(\mathfrak{n}_{v}\right)-\left(1+\left|\varpi_{v}\right|_{k}^{-1}\right) \sigma_{1}\left(\mathfrak{n}_{v} / \varpi_{v}\right)+\left|\varpi_{v}\right|_{k}^{-1} \sigma_{1}\left(\mathfrak{n}_{v} / \varpi_{v}^{2}\right) & \text { otherwise. }\end{cases}
$$

where $\sigma_{1}(\mathfrak{n})=\sum_{\mathfrak{m} \mid \mathfrak{n}} \mathrm{N}_{F / \mathbb{Q}}(\mathfrak{m})$.

Proposition 2.2.1. The following identity of cycles in $\mathcal{M}$ with rational coeffcients holds:

$$
\mathcal{M}_{c}=\mathrm{T}_{c}^{*} \mathcal{M}_{\min }
$$


Our plan to prove this Proposition is to express $T_{\mathfrak{n}} \mathcal{M}_{\text {min }}$ as a sum of $\mathcal{M}_{c}$ and try to convert the formula for any ideal $\mathfrak{n}$ of $\mathcal{O}_{F}$. Let $H_{\mathfrak{n}}$ denote the set of integral matrices in $M_{2}\left(\mathcal{O}_{F}\right)$ of determinant $\mathfrak{n}$ then we have an expression

$$
\mathrm{T}_{\mathfrak{n}} \mathcal{M}_{\text {min }}=\sum_{i} \mathcal{M}_{\tau g_{i}}
$$

where $\tau$ is an element in $G(\mathbb{A})$ with the Mumdord-Tate group $D^{\times} / k^{\times}$such that $\mathcal{M}_{\text {min }}=\mathcal{M}_{\tau}$ and $g_{i}$ are representatives of $H_{\mathfrak{n}} / H_{1}$. Thus the multiplicity $m(\mathfrak{n}, c)$ of $\mathcal{M}_{c}$ in $\mathrm{T}_{\mathfrak{n}} \mathcal{M}_{\text {min }}$ is the number of $\tau g_{i}$ with conductor $c$. Let $\Lambda_{i}$ be the submodule of $D$ such that

$$
(0,1) \Lambda_{i} \tau g_{i}=\mathcal{O}_{F}^{2}
$$

then $\Lambda_{i}$ is an invertible module over an order

$$
R_{i}=\left\{x \in D: \quad x \Lambda_{i} \in \Lambda_{i}\right\} .
$$

The conductor of $\tau g_{i}$ is the conductor of $R_{i}$. Recall that we may normalize $\tau$ by the following expression:

$$
(0,1) \mathcal{O}_{D} \tau=\mathcal{O}_{F}^{2},
$$

and thus we have

$$
(0,1) \mathcal{O}_{D} \tau g_{i}=\mathcal{O}_{F}^{2} g_{i} .
$$

Since $\mathcal{O}_{F}^{2} g_{i}$ runs through the submodules of $\mathcal{O}_{F}^{2}$ of index $\mathfrak{n}$, the modules $\Lambda_{i}$ will run through submodules of $\mathcal{O}_{D}$ of index $\mathfrak{n}$. Thus the multiplicity $m(\mathfrak{n}, c)$ of $\mathcal{M}_{c}$ in $T_{\mathfrak{n}} \mathcal{M}_{\text {min }}$ runs through the set of sublattices of $\mathcal{O}_{D}$ of index $\mathfrak{n}$ with conductor c.

For each ideal $\mathcal{N}$ of $\mathcal{O}_{F}$, let $R_{\mathcal{N}}$ denote the order

$$
R_{\mathcal{N}}=\mathcal{O}_{F}+\mathcal{N} \mathcal{O}_{D}
$$

and let $m(\mathfrak{n}, \mathcal{N})$ denote the number of $R_{\mathcal{N}}$-invertible module in $\mathcal{O}_{D}$ of determinant $\mathfrak{n}$. Then

$$
m(\mathfrak{n}, c)=\sum_{\mathrm{N}_{F / k}(\mathcal{N})=c} m(\mathfrak{n}, \mathcal{N}) .
$$

Note that for a fixed $\mathcal{N}$, an invertible $R$-submodule $\Lambda$ in $\mathcal{O}_{D}$ has the form $R_{\mathcal{N}} x:=\mathcal{O}_{D} \cap \widehat{R}_{\mathcal{N}} x$ with $x$ a nonzero element in $\widehat{\mathcal{O}}_{D}$. The element $x$ is uniquely determined up to multiplication by elements in $\widehat{R}_{\mathcal{N}}^{\times}$. The index of $R_{\mathcal{N}} x$ is given by

$$
\mathcal{N} \cdot \operatorname{det} x=\mathfrak{n} .
$$

Thus $m(\mathfrak{n}, \mathcal{N})$ is the number of elements in $\widehat{R}_{\mathcal{N}}^{\times} \backslash \widehat{\mathcal{O}}_{D}$ of determinant $\mathfrak{n} \mathcal{N}^{-1}$. This is the same as the product of $\left[\widehat{\mathcal{O}}_{D}^{\times}: \widehat{R}_{\mathcal{N}}^{\times}\right]$and the set of elements in $\widehat{\mathcal{O}}_{D}^{\times} \backslash \mathcal{O}_{D}$ with determinant $\mathfrak{n} \mathcal{N}^{-1}$.

For each $\mathfrak{n}$, we may write $\mathfrak{n}=\mathfrak{n}^{\prime} \mathfrak{n}^{\prime \prime}$ where $\mathfrak{n}^{\prime}$ is the maximal factor of $\mathfrak{n}$ in $\mathcal{O}_{k}$. Since $\operatorname{det} x \in \mathcal{O}_{k}$, the $\mathcal{N}$ must have the form $\mathfrak{n}^{\prime \prime} \mathcal{N}^{\prime}$ with $\mathcal{N}^{\prime}$ dividing $\mathfrak{n}^{\prime}$. 
Also since $\mathcal{N}$ is normalized with norm $c=c^{\prime \prime}\left(c^{\prime}\right)^{2}$, one must have $c^{\prime}=\mathcal{N}^{\prime}$ and $c^{\prime \prime}=\mathrm{N}_{F / k}\left(\mathfrak{n}^{\prime \prime}\right)$.

In summary, we have obtained that $T_{\mathfrak{n}} \mathcal{M}_{\text {min }}$ is the sum of $\mathcal{M}_{c}$ 's for $c$ having the form $c=\mathrm{N}_{F / k}\left(\mathfrak{n}^{\prime \prime}\right)\left(c^{\prime}\right)^{2}$ with $c^{\prime}$ dividing $\mathfrak{n}^{\prime}$. Moreover, the multiplicity of $\mathcal{M}_{c}$ in $\mathrm{T}_{\mathfrak{n}} \mathcal{M}_{\text {min }}$ is given by

$$
m(\mathfrak{n}, c)=\mu(c) \cdot \#\left\{x \in \widehat{\mathcal{O}}_{D}^{\times} \backslash \widehat{\mathcal{O}}_{D}, \operatorname{det} x=\mathfrak{n}^{\prime} / c^{\prime}\right\}
$$

where $\mu(c)$ is the index of $\widehat{R}^{\times}$in $\widehat{\mathcal{O}}_{D}$ and $R$ is an order of conductor $c$.

Now for an integer $d \geq 0$ and a finite place $v$ of $k$, let us compute the number of elements $x \in \mathcal{O}_{D_{v}}^{\times} \backslash \mathcal{O}_{D_{v}}$ with order $d$. If $D_{v}$ is not split, there is only one such element. If $D_{v}$ is split, then the number we want is

$$
\sigma_{1}\left(\varpi_{v}^{d}\right)=\sum_{i=0}^{d}\left|\varpi_{v}\right|^{-i}
$$

Thus we obtain

$$
\mathrm{T}_{\mathfrak{n}} \mathcal{M}_{\text {min }}=\sum_{c^{\prime} \mid \mathfrak{n}^{\prime}} \mu\left(\mathrm{N}_{F / k}\left(\mathfrak{n}^{\prime \prime}\right) c^{\prime 2}\right) \sigma_{1}^{D}\left(\mathfrak{n}^{\prime} / c^{\prime}\right) \mathcal{M}_{\mathrm{N}_{F / k}\left(\mathfrak{n}^{\prime \prime}\right) c^{\prime 2}}
$$

where $\sigma_{1}^{D}(\mathfrak{n})$ is computed only at places over which $D_{v}$ is split.

Now we try to convert this formula. The question is purely local. We fix a place $v$ of $k$ and consider ideals $\mathfrak{n}=\mathfrak{n}^{\prime} \mathfrak{n}^{\prime \prime}$ of $\mathcal{O}_{F, v}$ with $\mathfrak{n}^{\prime \prime}$ fixed and $\mathfrak{n}^{\prime}=\varpi_{v}^{i}$ running through all ideals of $\mathcal{O}_{k, v}$. Now consider formal power series of $t$ :

$$
\sum_{i} \mathrm{~T}_{\mathfrak{n}^{\prime \prime} \varpi_{v}^{i}} \mathcal{M}_{m i n} \cdot t^{i}=\sum_{m} \sigma_{1}^{D}\left(\varpi_{v}^{m}\right) t^{m} \sum_{j} \mu\left(\mathrm{N}_{F / k}\left(\mathfrak{n}^{\prime \prime}\right) \varpi_{v}^{2 j}\right) \cdot \mathcal{M}_{\mathrm{N}_{F / k}\left(n^{\prime \prime}\right)\left(\varpi_{v}\right)^{2 j}} \cdot t^{j} .
$$

Notice that

$$
\sum_{m} \sigma_{1}^{D}\left(\varpi_{v}^{m}\right) t^{m}=\left\{\begin{array}{ll}
\left(1-\left|\varpi_{i}\right|^{-1} t\right)^{-1}(1-t)^{-1} & \text { if } v \mid \delta_{D} \\
1 & \text { otherwise }
\end{array} .\right.
$$

We thus obtain

$$
\begin{aligned}
\sum_{j} \mu\left(\mathrm{N}_{F / k}\left(\mathfrak{n}^{\prime \prime}\right) \varpi^{2 j}\right) \cdot \mathcal{M}_{\mathrm{N}_{F / k}\left(n^{\prime \prime}\right) \varpi^{2 j}} \cdot t^{j}= & \sum_{i} \mathrm{~T}_{n^{\prime \prime} \varpi^{i}} \mathcal{M}_{m i n} \cdot t^{i} \\
& \cdot \begin{cases}\left(1-\left|\varpi_{i}\right|^{-1} t\right)(1-t) & \text { if } v \mid \delta_{D} \\
1 & \text { otherwise }\end{cases}
\end{aligned}
$$

It follows that

$$
\begin{aligned}
& \mu\left(\mathrm{N}_{F / k}\left(\mathfrak{n}^{\prime \prime}\right) \varpi^{2 j}\right) \cdot \mathcal{M}_{\mathrm{N}_{F / k}\left(\mathfrak{n}^{\prime \prime}\right) \varpi^{2 j}} \\
= & \begin{cases}\mathrm{T}_{\mathfrak{n}^{\prime \prime} \varpi^{j}} \mathcal{M}_{\text {min }} & \text { if } v \mid \delta_{D} \\
\mathrm{~T}_{\mathfrak{n}^{\prime \prime} \varpi^{j}} \mathcal{M}_{\text {min }}-\left(1+\left|\varpi_{v}\right|^{-1}\right) \mathrm{T}_{\mathfrak{n}^{\prime \prime} \varpi^{j-1}} \mathcal{M}_{\text {min }}+\left|\varpi_{v}\right|^{-1} \mathrm{~T}_{\mathfrak{n}^{\prime \prime} \varpi^{j-2}} \mathcal{M}_{\text {min }}, & \text { otherwise. }\end{cases}
\end{aligned}
$$


This completes the proof of Proposition 2.2.1.

2.3. From Theorem 1.2.1 to Theorem 1.2.3. In this section we show that Theorem 1.2.3 follows from Theorem 1.2.1. Let $\varphi$ be a smooth function on $\mathcal{M}^{0}$ with compact support. Let $\mathcal{M}_{x}$ be a sequence of Shimura subvarieties in $\mathcal{M}$ defined by datum $(D, c)$ where $D$ is a quaternion algebra over $k$ and $c$ is the conductor of $\mathcal{M}_{x}$. Assume that the intersection $\mathcal{M}_{x}^{0}$ of $\mathcal{M}_{x}$ with $\mathcal{M}^{0}$ is nonempty. We need to estimate the integral $\ell_{\mathcal{M}_{x}^{0}}(\varphi)$. We may extend $\varphi$ to all of $\mathcal{M}$ by setting $\varphi=0$ at other components and write

$$
\ell_{\mathcal{M}_{x}^{0}}(\varphi)=\left(\# \pi_{0}(\mathcal{M})\right)^{-1} \sum_{\alpha} \ell_{\mathcal{M}_{x}}(\varphi \alpha)
$$

where the summation runs through the characters $\alpha$ of the group of the connected components $\pi_{0}(\mathcal{M})$ which are also considered as functions on $\mathcal{M}$, and

$$
\ell_{\mathcal{M}_{x}}(\varphi)=\operatorname{vol}\left(\mathcal{M}_{x}\right)^{-1} \int_{\mathcal{M}_{x}} \varphi(h) d h
$$

Thus the question is reduced to estimate $\ell_{\mathcal{M}_{x}}(\varphi)$. Here $\varphi$ is identified with the spherical function (also denoted by $\varphi$ ) on $\mathbf{G}(\mathbb{Q}) \backslash \mathbf{G}(\mathbb{A})$.

Consider the spectral decomposition:

$$
\varphi=\sum c_{n} \varphi_{n}+\int_{\Omega} c_{\mu} E_{\mu} d \mu
$$

where $\varphi_{n}$ 's are discrete (cuspidal or residual) eigenform under Hecke operators with norm 1 , and $\mu$ runs through the set $\Omega$ of characters of $F^{\times} \backslash \mathbb{A}_{F}^{\times}$modulo equivalence $\mu \sim \mu^{-1}$, and $E_{\mu}$ is certain form of norm 1 .

For the discrete spectrum, we may take $\varphi_{n}$ to be characters of $\mathbf{G}(\mathbb{Q}) \backslash \mathbf{G}(\mathbb{A})$, or

$$
\varphi_{n}=\left\|\varphi_{n}^{\text {new }}\right\|^{-1} \varphi_{n}^{\text {new }}
$$

with $\varphi_{n}^{\text {new }}$ newforms. For the continuous spectrum, we may take $E_{\mu}$ to be

$$
E_{\mu}=\left|L\left(1, \mu^{2}\right)\right|^{-1} E_{\mu}^{\text {new }}
$$

where $E_{\mu}^{\text {new }}$ is the newform in $\pi\left(\mu, \mu^{-1}\right)$. The measure $d \mu$ is induced from a Haar measure on the topological group of idele class character of $F$. Since $\varphi$ is compactly supported we have

$$
\|\varphi\|^{2}=\sum\left|c_{n}\right|^{2}+\int_{\Omega}\left|c_{\mu}\right|^{2} d \mu<\infty .
$$

Moreover, $\Delta^{N} \varphi$ is still compactly supported,

$$
\left\|\Delta^{N} \varphi\right\|^{2}=\sum\left|c_{n} \lambda_{n}^{N}\right|^{2}+\int_{\Omega}\left|c_{\mu} \lambda_{\mu}^{N}\right|^{2} d \mu<\infty
$$

where $\lambda_{i}$ (resp. $\lambda_{\mu}$ ) are eigenvalues of $\varphi_{i}$ (resp. $E_{\mu}$ ) under $\Delta$. Thus $c_{n}$ (resp. $c_{\mu}$ ) decays faster than any negative power of $\lambda_{n}$ (resp. $\left.\lambda_{\mu}\right)$. It can be shown that 
$\left\|\phi_{n}\right\|_{\text {sup }}$ is bounded by a polynomial function of $\lambda_{n}$. Thus, the sum of the right hand side of (2.3.2) is absolutely convergent point-wisely. Similarly, for a fixed compact domain $E$ of $\mathcal{M}(\mathbb{C})$, it can be shown that $\sup _{x \in E}\left|E_{\mu}(x)\right|$ is bounded by a polynomial function of $\lambda_{\mu}$. Thus, the integral of the right hand side of (2.3.2) are absolutely and uniformly convergent on $E$. See Clozel-Ullmo [4], Lemma 7.2-7.4 for a complete proof. It follows for each compact $\mathcal{M}_{x}$ that

$$
\ell_{\mathcal{M}_{x}}(\varphi)=\sum c_{n} \ell_{\mathcal{M}_{x}}\left(\varphi_{n}\right)+\int_{\Omega} c_{\mu} \ell_{\mathcal{M}_{x}}\left(E_{\mu}\right) d \mu
$$

Thus it suffices to show the following:

Proposition 2.3.1. For any $\varepsilon>0$, there are positive numbers $C$ and $N$ such that for any new form $\varphi$ (which is either cuspidal or Eisenstein):

$$
\left|\ell_{\mathcal{M}_{D, c}}(\varphi)\right| \leq C \cdot\|\varphi\| \cdot \lambda(\varphi)^{N} \cdot \mathrm{N}_{k / \mathbb{Q}}\left(\delta_{D, c}\right)^{\varepsilon+\alpha-1 / 2}
$$

where

- $\|\varphi\|=\left|L\left(1, \mu^{2}\right)\right|$ if $\varphi \in \pi\left(\mu, \mu^{-1}\right)$,

- $\alpha$ is a positive number such that

$$
\left|\chi_{v}\left(\varpi_{v}\right)\right|<<\left|\varpi_{v}\right|^{-\alpha}
$$

for every finite place $v$ where $\chi_{v}$ is an unramified character of $k_{v}^{\times}$such that $\pi_{k, v}=\left(\chi_{v}, \chi_{v}^{-1} \eta_{v}\right)$.

It remains to prove this Proposition. By Proposition 2.2.1 we have

$$
\ell_{\mathcal{M}_{D, c}}(\varphi)=\lambda_{\varphi}\left(\mathrm{T}_{c}^{*}\right) \ell_{\mathcal{M}_{D, 1}}(\varphi)
$$

where $\lambda_{\varphi}\left(\mathrm{T}_{c}^{*}\right)$ is the eigenvalue of $\varphi$ under the Hecke operator $\mathrm{T}_{c}^{*}$. Now the question is reduced to the estimate of $\lambda_{\varphi}\left(\mathrm{T}_{c}^{*}\right)$ and $\ell_{\mathcal{M}_{D, 1}}(\varphi)$.

By Theorem 1.2.1 and property of $\alpha$ in the Proposition, we have

$$
\left|\ell_{\mathcal{M}_{D, 1}}(\varphi)\right|<<\mathrm{N}_{k / \mathbb{Q}}\left(\delta_{D, 1}\right)^{\varepsilon+\alpha-1 / 2} \lambda(\varphi)^{\varepsilon} .
$$

It remains to estimate $\lambda_{\varphi}\left(\mathrm{T}_{c}^{*}\right)$. Notice that the eigenvalues of $\varphi$ under $\mathrm{T}_{n}$ is given by $a_{n}|n|^{-1 / 2}$, where $a_{n}$ is the $n$-Fourier coefficient of $f$ which is bounded by $\mathrm{N}_{F / \mathbb{Q}}(n)^{\alpha+1 / 2}$. It follows that

$$
\left|\lambda_{\varphi}\left(\mathrm{T}_{c}^{*}\right)\right|<<\mathrm{N}_{k / \mathbb{Q}}(c)^{\epsilon+\alpha-1 / 2} .
$$

This proves the proposition.

Notice that we can forget $\|\varphi\|$ in Proposition 2.3.1, because the following estimate is well known:

$$
\lambda(\varphi)^{\varepsilon}>>\|\varphi\|>>\lambda(\varphi)^{-\varepsilon}
$$

for $\lambda$ large and any $\varepsilon>0$. 


\section{Periods of Eisenstein series}

This section is devoted to the proof of Theorem 1.2.1 for Eisenstein series. After a direct computation, the period with respect to a standard measure is expressed as a product of local integrals of Godement-Jacquet type. From this expression we obtain the volumes of the cycles and deduce Theorem 1.2.1 for Eisenstein series.

3.1. First computations. We are going to study the nonvanishing condition and its explicit formula for the following period integral

$$
\int_{H(k) \backslash H\left(\mathbb{A}_{k}\right)} \varphi(h \tau) d h
$$

with $\varphi$ being an Eisenstein series on $\mathbf{G}(\mathbb{A})=\operatorname{PGL}_{2}\left(\mathbb{A}_{F}\right)$. Here recall that $H=$ $D^{\times} / k^{\times}$with $D=F+F \lambda$ a quaternion algebra over $k$ embedded into $M_{2}(F)$ by $\iota$ defined in (2.1.1) with $\gamma=1$ :

$$
\iota(x+y \lambda)=\left(\begin{array}{ll}
x & y \epsilon \\
\bar{y} & \bar{x}
\end{array}\right) .
$$

Before we begin our explicit computation, let us fix the standard measures on various groups as follows.

(1) For $\mathrm{GL}_{1}\left(\mathbb{A}_{F}\right)$ (or $\mathrm{GL}_{1}\left(\mathbb{A}_{k}\right)$ ), we choose a measure $d^{\times} t=\otimes d^{\times} t_{v}$ such that $\operatorname{vol}\left(\mathcal{O}_{F, v}^{\times}\right)=1$ for all finite places and such that $d^{\times} x=d x / x$ at archimedean places.

(2) For $\mathrm{GL}_{2}\left(\mathbb{A}_{F}\right)$ (or $D^{\times}\left(\mathbb{A}_{k}\right)$ ) we choose measures with local decomposition $d g=\otimes d g_{v}$ such that at a finite place, the maximal compact subgroup has volume 1 . At an infinite place $v$ with coordinates given by the Iwasawa decomposition

$$
g=\left(\begin{array}{ll}
z & 0 \\
0 & z
\end{array}\right)\left(\begin{array}{ll}
y & x \\
0 & 1
\end{array}\right) k, \quad k \in \mathrm{SO}_{2}(\mathbb{R})
$$

we take

$$
d g=d^{\times} z \cdot d^{\times} y \cdot\left|y^{-1} d x\right| \cdot d k
$$

where $d^{\times} x$ is the Harr measure $d x / x$ on $\mathbb{R}^{\times}, d k$ is a Haar measure on $\mathrm{SO}_{2}(\mathbb{R})$ of volume 1 .

In this section we write $\mathbf{G}(\mathbb{Q})=\mathrm{PGL}_{2}(F)$ and $B(F)=T(F) N(F)$ the standard Borel subgroup of $\mathrm{PGL}_{2}(F)$ with $T$ the diagonal matrices, and $N$ the unipotent subgroup. Let $\mu$ be a character of $\mathbb{A}_{F}{ }^{\times} / F^{\times}$, which is also a character of $T\left(\mathbb{A}_{F}\right) / T(F)$ : if $m=\left(\begin{array}{ll}a & 0 \\ 0 & b\end{array}\right)$, then we have

$$
\mu(m):=\mu\left(\frac{a}{b}\right) .
$$


We write $|m|$ for the character $|a / b|$. We consider the induced representation

$$
I(\mu)=\operatorname{Ind}_{B\left(\mathbb{A}_{F}\right)}^{\mathrm{PGL}_{2}\left(\mathbb{A}_{F}\right)}(\mu),
$$

which consists of smooth complex functions $f$ on $\mathrm{PGL}_{2}\left(\mathbb{A}_{F}\right)$ satisfying the following property:

$$
f(b g)=\mu(m)|m|^{1 / 2} \cdot f(g) .
$$

We define an Eisenstein series for each $f \in I(\mu)$ by

$$
E(g ; \mu, f)=\sum_{\gamma \in B(F) \backslash \mathrm{PGL}_{2}(F)} f(\gamma g) .
$$

It is known that the Eisenstein series converges absolutely for the real part of $\mu$ large and has a meromorphic continuation to the whole domain of $\mu$. Now we take $\varphi$ to be $E(g ; \mu, f)$ in the integral (3.1.1). We need the following decomposition:

Lemma 3.1.1. Let $B$ be the standard Borel subgroup of $\mathrm{GL}_{2}$, then

$$
\mathrm{GL}_{2}(F)=B(F) \cdot D^{\times} \text {. }
$$

if $D$ is non-split; and

$$
\mathrm{GL}_{2}(F)=B(F) \cdot D^{\times} \coprod B(F) \alpha D^{\times}
$$

if $D$ is split. Here $\alpha$ is an element such that

$$
\alpha D \alpha^{-1}=M_{2}(k) .
$$

Proof. Let $g=\left(\begin{array}{ll}a & b \\ c & d\end{array}\right)$ be an element in $\mathrm{GL}_{2}(F)$. We try to find an $h=\left(\begin{array}{ll}x & y \epsilon \\ \bar{y} & \bar{x}\end{array}\right)$ in $D^{\times}$such that $g h$ is triangular. We may assume that $c=1$. In this case, we may take $h$ with $x=d$ and $y=-1$. This element has determinant $d \bar{d}-\epsilon$ which is non split if $\epsilon$ is not a norm from $F$. For split group $\mathrm{GL}_{2}(k)$, one has

$$
\mathrm{GL}_{2}(F)=B(F) \cdot \mathrm{GL}_{2}(k) \coprod B(F) \cdot h \cdot \mathrm{GL}_{2}(k)
$$

where $h$ is any element not in

$$
B(F) \cdot \mathrm{GL}_{2}(k)=B(F) \cdot \alpha D^{\times} \alpha^{-1} .
$$

In other words,

$$
\mathrm{GL}_{2}(F)=B(F) \cdot h D^{\times} \coprod B(F) \cdot h \cdot \alpha D^{\times} .
$$

The lemma follows by taking $h=\alpha^{-1}$.

By this lemma, we have that the natural embedding $H \longrightarrow G$ induces a bijection

$$
B(F) \backslash \mathrm{PGL}_{2}(F) \simeq(B(F) \cap H(k)) \backslash H(k) \simeq F^{\times} \backslash D^{\times}
$$


if $D$ is non split; and

$$
B(F) \backslash \mathrm{PGL}_{2}(F) \simeq F^{\times} \backslash D^{\times} \coprod \alpha \cdot B_{H}(k) \backslash H(k)
$$

if $D$ is split, where $B_{H}$ is a Borel subgroup of $H$. Thus when the real part of $\mu$ is large and $D$ is nonsplit, we have

$$
\begin{aligned}
\int_{H(k) \backslash H\left(\mathbb{A}_{k}\right)} E(h \tau ; \mu, f) d h & =\int_{H(k) \backslash H\left(\mathbb{A}_{k}\right)} \sum_{\gamma \in B(F) \backslash \mathrm{PGL}_{2}(F)} f(\gamma h \tau) d h \\
& =\int_{F^{\times} \mathbb{A}_{k}^{\times} \backslash D^{\times}\left(\mathbb{A}_{k}\right)} f(h \tau) d h .
\end{aligned}
$$

We assume that $\mu=\mu_{0} \cdot|\cdot|_{\mathbb{A}_{F}}^{s}$. For any $a \in F^{\times}$, we have

$$
f(\iota(a) g)=\mu_{0}\left(\frac{a}{\bar{a}}\right) f(g) .
$$

It follows that the last integral in (3.1.4) can be written as follows:

$$
\begin{aligned}
\int_{F^{\times} \mathbb{A}_{k}^{\times} \backslash D^{\times}\left(\mathbb{A}_{k}\right)} f(h \tau) d h & =\int_{\mathbb{A}_{F}^{\times} \backslash D^{\times}\left(\mathbb{A}_{k}\right)} \int_{F^{\times} \mathbb{A}_{k}^{\times} \backslash \mathbb{A}_{F}^{\times}} f(t h \tau) d t d h \\
& =\int_{\mathbb{A}_{F}^{\times} \backslash D^{\times}(\mathbb{A})} f(h \tau) d h \int_{\mathbb{A}_{k}^{\times} \cdot F^{\times} \backslash \mathbb{A}_{F}^{\times}} \mu\left(\frac{a}{\bar{a}}\right) d^{\times} a .
\end{aligned}
$$

It is clear that

$$
C(\mu)=\int_{\mathbb{A}_{k}^{\times} \cdot F^{\times} \backslash \mathbb{A}_{F}^{\times}} \mu\left(\frac{a}{\bar{a}}\right) d^{\times} a
$$

is a well defined constant depending on the character $\mu$. Note that $C(\mu)$ vanishes if and only if $\mu(\alpha) \neq \mu(\bar{\alpha})$, i.e. the character $\mu$ is not stable under the Galois twist of $\operatorname{Gal}(F / k)$.

When $D$ is split, then we have $G(\mathbb{Q})=P G L_{2}(F)$ and $H(\mathbb{Q})=G L_{2}(k)$. The period of the Eisenstein series

$$
\int_{H(\mathbb{Q}) \backslash H(\mathbb{A})} E(h \tau ; \mu, f) d h
$$

can be regularized as in [12]. It was calculated explicitly in Example 6 on Page 238 of [12] that the regularized period of $E(h \tau ; \mu, f)$ is equal to

$$
\int_{F^{\times} \mathbb{A}_{k}^{\times} \backslash D^{\times}\left(\mathbb{A}_{k}\right)} f(h \tau) d h
$$

Thus we reach the following:

Lemma 3.1.2. The period

$$
\int_{H(k) \backslash H\left(\mathbb{A}_{k}\right)} E(h \tau ; \mu, f) d h
$$


does not vanish, i.e. $E(h \tau ; \mu, f)$ is $H\left(\mathbb{A}_{k}\right)$-distinguished for embedding defined by $(\iota, \tau)$ only if $\mu$ is $\operatorname{Gal}(F / k)$-stable; in this case it equals to

$$
\operatorname{vol}\left(\mathbb{A}_{k}^{\times} F^{\times} \backslash \mathbb{A}_{F}^{\times}\right) \int_{\mathbb{A}_{F}^{\times} \backslash D^{\times}\left(\mathbb{A}_{k}\right)} f(h \tau) d h .
$$

This is in fact expected from the general theory of quadratic base change from $k$ to $F$ for $\mathrm{GL}_{2}$. More precisely, $\pi\left(\mu, \mu^{-1}\right)$ is the base change of the representation $\pi\left(\chi, \chi^{-1} \eta\right)$ on $\mathrm{GL}_{2}\left(\mathbb{A}_{k}\right)$ when $\chi$ is the character on $k^{\times} \backslash \mathbb{A}_{k}^{\times}$such that $\mu(x)=$ $\chi \cdot \mathrm{N}_{F / k}(x)$.

3.2. Godement-Jacquet integrals. In the following we assume that the character $\mu$ is $\operatorname{Gal}(F / k)$-stable. Since $E(g, \mu, f)$ is spherical, one must have that $\mu$ is unramified at all finite places, and up to a constant multiple, $f=\mu^{-1}\left(\delta_{F}\right) f_{\phi}$ for a standard Bruhat-Schwartz function $\phi$ in $\mathcal{S}\left(\mathbb{A}_{F}^{2}\right)$ :

$$
f_{\phi}(g)=\mu(\operatorname{det}(g)) \cdot|\operatorname{det}(g)|_{\mathbb{A}_{F}}^{\frac{1}{2}} \cdot \int_{\mathbb{A}_{F}^{\times}} \phi((0, t) g) \mu^{2}(t)|t|_{\mathbb{A}_{F}} d^{\times} t .
$$

Here $\phi=\otimes \phi_{v}$ is decomposable with $\phi_{v}$ given as follows:

- for $v$ a finite place of $k, \phi_{v}$ is the characteristic function of $\mathcal{O}_{v}^{2}$.

- for $v$ an archimedean place of $F, \phi_{v}(x, y)=e^{-\pi\left(x^{2}+y^{2}\right)}$.

It is easy to check that $f_{\phi}$ is left $\mathbb{A}_{F}^{\times}$-invariant since $\mu$ is $\operatorname{Gal}(F / k)$-stable. Now we compute for the section $f_{\phi}$ the local period integral in Lemma 3.1.2.

$$
\begin{aligned}
\int_{\mathbb{A}_{F}^{\times} \backslash D^{\times}\left(\mathbb{A}_{k}\right)} f_{\phi}(h \tau) d h= & \int_{\mathbb{A}_{F}^{\times} \backslash D^{\times}\left(\mathbb{A}_{k}\right)} \mu(\operatorname{det}(h \tau)) \cdot|\operatorname{det}(h \tau)|_{\mathbb{A}_{F}}^{\frac{1}{2}} . \\
& \cdot \int_{\mathbb{A}_{F}^{\times}} \phi((0, t) h \tau) \mu^{2}(t)|t|_{\mathbb{A}_{F}} d^{\times} t d h .
\end{aligned}
$$

By changing the variables, the last integral is equal to

$$
\int_{D^{\times}\left(\mathbb{A}_{k}\right)} \phi((0,1) h \tau) \mu(\operatorname{det}(h \tau))|\operatorname{det}(h \tau)|_{\mathbb{A}_{F}}^{\frac{1}{2}} d h .
$$

Therefore, we obtain

Lemma 3.2.1. Let $\varphi$ be a Bruhat-Schwartz function on $D(\mathbb{A})$ defined by

$$
\varphi(h)=\phi((0,1) h \tau) .
$$

Let $\chi$ be a character of $k^{\times} \backslash \mathbb{A}_{k}^{\times}$such that $\mu=\chi \circ \mathrm{N}_{F / k}$. Then

$$
\int_{\mathbb{A}_{F}^{\times} \backslash D^{\times}\left(\mathbb{A}_{k}\right)} f_{\phi}(h) d h=\chi\left(\mathrm{N}_{F / k} \operatorname{det} \tau\right)|\operatorname{det} \tau|_{F}^{1 / 2} Z\left(\chi^{2}, \varphi\right)
$$


where $Z\left(\chi^{2}, \varphi\right)$ is the Godement-Jacquet integral:

$$
Z\left(\chi^{2}, \varphi\right):=\int_{D^{\times}\left(\mathbb{A}_{k}\right)} \varphi(h) \chi^{2}(\operatorname{det}(h))|\operatorname{det}(h)|_{k} d h .
$$

Note that this last integral is a special case $(n=d=2)$ studied in GodementJacquet's book [7], Proposition 4.4. This integral is a product of the corresponding local integrals $Z\left(\mu_{v}, \varphi_{v}\right)$ with respect to a decomposition of measures $d h=\otimes d h_{v}$. In the following we want to evaluate this integral for the case where $\tau$ satisfies conditions (2.1.2) and (2.1.3) for trivial ideal $\mathcal{N}=\mathcal{O}_{F}$. We normalize the measure $d x_{v}$ which is standard at achimedean place and that $\operatorname{vol}\left(\mathcal{O}_{D, v}\right)=1$ at finite places $v$ where $\mathcal{O}_{D}$ is a maximal order of $D$.

Lemma 3.2.2. Let $v$ be a finite place of $k$. Then

$$
Z\left(\chi_{v}^{2}, \varphi_{v}\right)= \begin{cases}L\left(1, \chi_{v}^{2}\right) L\left(0, \chi_{v}^{2}\right), & \text { if } D_{v} \text { is split } \\ L\left(1, \chi_{v}^{2}\right), & \text { otherwise. }\end{cases}
$$

Proof. By our assumption (2.1.3), $\varphi_{v}$ is the characteristic function of $\mathcal{O}_{D, v}$, a maximal order of $\mathcal{O}_{v}$. It follows that

$$
Z\left(\chi_{v}^{2}, \varphi_{v}\right)=\sum_{n \geq 0} \operatorname{vol}\left(\mathcal{O}_{v}(n)\right) \chi_{v}^{2}(\varpi)^{n}|\varpi|^{n}
$$

where $\mathcal{O}_{v}(n)$ is the set of integral matrices in $\mathcal{O}_{D, v}$ whose determinant have order $n$.

If $D_{v}$ is not split, then $\mathcal{O}_{D, v}(n)=\varpi_{D_{v}}^{n} \mathcal{O}_{D, v}^{\times}$. It follows that

$$
Z\left(\chi_{v}, \varphi_{v}\right)=\sum_{n \geq 0} \chi_{v}^{2}(\varpi)^{n}|\varpi|^{n}=L\left(1, \chi_{v}^{2}\right) .
$$

Now assume that $D_{v}$ is split. Then there is an isomorphism $D_{v} \simeq M_{2}\left(k_{v}\right)$ such that $\mathcal{O}_{v} \simeq M_{2}\left(\mathcal{O}_{k_{v}}\right)$. It is well-know that $\mathcal{O}_{v}(n)$ has decomposition into $\mathcal{O}_{D, v}^{\times}$-coset:

It follows that

$$
\mathcal{O}_{v}(n)=\coprod_{\substack{i+j=n \\
x \bmod \varpi^{i}}}\left(\begin{array}{cc}
\varpi^{i} & x \\
0 & \varpi^{j}
\end{array}\right) \mathcal{O}_{D, v}^{\times} .
$$

$$
\begin{gathered}
\operatorname{vol}\left(\mathcal{O}_{v}(n)\right)=\sum_{i+j=n}|\varpi|^{-i} \\
Z\left(\chi_{v}^{2}, \varphi_{v}\right)=\sum_{n \geq 0} \sum_{i+j=n} \chi_{v}^{2}(\varpi)^{i+j}|\varpi|^{j}=L\left(0, \chi_{v}^{2}\right) L\left(1, \chi_{v}^{2}\right) .
\end{gathered}
$$

It remains to compute the integral at an archimedean place $v$. 
Lemma 3.2.3. Let $v$ be an archimedean place, then

$$
Z\left(\chi_{v}^{2}, \varphi_{v}\right)=L\left(0, \chi_{v}^{2}\right) L\left(1, \chi_{v}^{2}\right) .
$$

Proof. In this case, $F_{v}=\mathbb{R}^{2}$, and the subalgebra $D_{v}$ is split and consists of matrices

$$
h=\left(\begin{array}{ll}
a & b \epsilon \\
\bar{b} & \bar{a}
\end{array}\right)=\left(\left(\begin{array}{ll}
\alpha \beta \\
\gamma & \delta
\end{array}\right), \quad\left(\begin{array}{cc}
\delta & \gamma \epsilon \\
\beta \epsilon^{-1} & \alpha
\end{array}\right)\right) .
$$

Take

The function $\varphi$ is given by

$$
\tau_{v}=\left(1, \quad\left(\begin{array}{ll}
0 & \epsilon \\
1 & 0
\end{array}\right)\right) .
$$

$$
\left.\varphi(h)=\phi\left((0,1) h \tau_{v}\right)\right)=e^{-\pi Q(h)}
$$

where for $h=\left(\begin{array}{l}\alpha \beta \\ \gamma \delta\end{array}\right)$

$$
Q(x)=\alpha^{2}+\beta^{2}+\gamma^{2}+\delta^{2} .
$$

Thus

$$
Z\left(\chi^{2}, \varphi\right)=\int_{\mathrm{GL}_{2}(\mathbb{R})} e^{-\pi Q(h)} \chi^{2}(\operatorname{det}(h))|\operatorname{det} h| d h .
$$

Notice that $Q(h)$ does not change under the translation of $U_{v}:=\mathrm{SO}_{2}(\mathbb{R})$. Thus we have

$$
\begin{aligned}
Z(\chi, \varphi) & =\int_{B(\mathbb{R})} e^{-\pi Q(b)} \chi^{2}(\operatorname{det}(b)) \cdot|\operatorname{det}(b)| d b \\
& =\int e^{-\pi t^{2}\left(1+x^{2}+y^{2}\right)} \chi_{v}^{2}\left(t^{2} y\right)\left|t^{2} y\right||y|^{-1} d x d^{\times} y d^{\times} t \\
& =L\left(0, \chi_{v}^{2}\right) L\left(1, \chi_{v}^{2}\right)
\end{aligned}
$$

To compute the integral in Lemma 3.2.1, it remains to compute $\mathrm{N}_{F / k}(\operatorname{det} \tau)$.

Lemma 3.2.4. Assume that conditions (2.1.2) and (2.1.3) hold for trivial ideal $\mathcal{N}=\mathcal{O}_{F}$. Let $\delta_{F}, \delta_{D} \in \mathbb{A}_{k}^{\times}$be ideles with trivial infinite components such that their finite components generate the relative discriminants of $F$ and $D$ over $k$. Then we have

$$
\mathrm{N}_{F / k}(\operatorname{det}(\tau)) \equiv-\epsilon \delta_{F} \delta_{D}^{-1} \bmod \widehat{\mathcal{O}}_{k}^{\times}
$$

Proof. Obviously, by (2.1.2), there is nothing to prove for archimedean places. So we need only treat the nonarchimedean places. We identify $D$ with $F^{2}$ by the isomorphism

$$
x \in D \longrightarrow(0,1) x \in F^{2} .
$$


Thus the trivial lattice $\mathcal{O}_{F}^{2}$ in $F^{2}$ corresponds to the lattice $\mathcal{O}_{F}+\mathcal{O}_{F} \cdot \lambda$. By (2.1.3),

$$
\mathcal{O}_{D}=\left(\mathcal{O}_{F}+\mathcal{O}_{F} \lambda\right) \tau^{-1}
$$

Recall that the discriminant $\operatorname{disc}\left(\mathcal{O}_{D}\right)$ is a fractional ideal of $k$ such that

$$
\operatorname{det}\left(\mathcal{O}_{D}\right)=\operatorname{disc}\left(\mathcal{O}_{D}\right)^{2} \operatorname{det}\left(\mathcal{O}_{D}^{\vee}\right)
$$

in $\operatorname{det}(D)=\wedge^{4}(D)$, where $\mathcal{O}_{D}^{\vee}$ is the dual of $\mathcal{O}_{D}$ with respect to the following pairing on $D$ :

$$
(x, y)=\operatorname{tr}_{D / k}(x \bar{y}) .
$$

We want to compute the discriminant for $\mathcal{O}_{D}$ by the above explicit description. Thus we need to compare $\operatorname{det} \mathcal{O}_{D}$ and $\operatorname{det} \mathcal{O}_{D}^{\vee}$. Since $\mathcal{O}_{D}$ is a module over $\mathcal{O}_{F}$, we may identify

$$
\operatorname{det} \mathcal{O}_{D}=\mathrm{N}_{F / k}\left(\operatorname{det}_{F} \mathcal{O}_{D}\right)
$$

where $\operatorname{det}_{F}$ means the determinant of $\mathcal{O}_{D}$ as $\mathcal{O}_{F}$-module, and $\mathrm{N}_{F / k}\left(\operatorname{det}_{F} \mathcal{O}_{D}\right)$ is an $\mathcal{O}_{F}$-module generated by symbols $\mathrm{N}_{F / k}(t)$ for $t \in \operatorname{det}_{F} \Lambda$ with relation

$$
\mathrm{N}_{F / k}(a t)=\mathrm{N}_{F / k}(a) \mathrm{N}_{F / k}(t), \quad a \in \mathcal{O}_{F} .
$$

It is easy to see that in this identification:

$$
\operatorname{det} \mathcal{O}_{D}=\mathcal{O}_{F} \mathrm{~N}_{F / k}(\operatorname{det} \tau)^{-1} \mathrm{~N}_{F / k}(1 \wedge \lambda) \text {. }
$$

It remains to compute $\mathcal{O}_{D}^{\vee}$ and $\operatorname{det} \mathcal{O}_{D}^{\vee}$. We will use the following identity:

$$
(x, y)=\operatorname{tr}_{F / k}(x, y)_{F}
$$

with

$$
(x, y)_{F}=\rho_{F}(x \bar{y})
$$

where $\rho_{F}$ is the projection of $D$ onto $F$ with respect to the decomposition:

$$
D=F+F \lambda .
$$

Then, for $x \in D, x \in \mathcal{O}_{D}^{\vee}$ if and only if $\operatorname{tr}\left(x \mathcal{O}_{D}\right)$ is integral, and the later is equivalent to that

$$
\rho_{F}(\bar{x} \Lambda)_{F} \subset \mathcal{O}_{F}^{\vee}
$$

where $\mathcal{O}_{F}^{\vee}$ is the dual of $\mathcal{O}_{F}$ with respect to $\operatorname{tr}_{F / k}$. Thus we have

$$
\mathcal{O}_{D}^{\vee}=\mathcal{O}_{F}^{\vee} \cdot \mathcal{O}_{D}^{*}
$$

where $\mathcal{O}_{D}^{*}$ is the dual of $\mathcal{O}_{D}$ with respect to $(\cdot, \cdot)_{F}$.

Let $\tau^{*}$ denote the adjoint of $\tau$ with respect to $(\cdot, \cdot)_{F}$ :

$$
(x \tau, y)_{F}=\left(x, y \tau^{*}\right)_{F} .
$$

Then

$$
\mathcal{O}_{D}^{*}=\left(\mathcal{O}_{F}+\mathcal{O}_{F} \epsilon^{-1} \lambda\right) \tau^{*}
$$


Thus we obtain

$$
\mathcal{O}_{D}^{\vee}=\mathcal{O}_{F}^{\vee} \cdot\left(\mathcal{O}_{F}+\epsilon^{-1} \mathcal{O}_{F} \lambda\right) \cdot \tau^{*}
$$

It follows that

$$
\begin{aligned}
\operatorname{det} \mathcal{O}_{D}^{\vee} & =\mathrm{N}_{F / k}\left(\operatorname{det} \mathcal{O}_{D}^{\vee}\right)=\mathrm{N}_{F / k}\left(\left(\mathcal{O}_{F}^{\vee}\right)^{2} \cdot \epsilon^{-1} \operatorname{det}\left(\tau^{*}\right)(1 \wedge \lambda)\right) \\
& =\mathcal{O}_{k, v} \epsilon^{-1} \delta_{F / k}^{-2} \mathrm{~N}(\operatorname{det} \tau) \mathrm{N}(1 \wedge \lambda) .
\end{aligned}
$$

Now (3.2.3) and (3.2.4) imply that

$$
\operatorname{disc}\left(\mathcal{O}_{D}\right)=\epsilon \delta_{F} \mathrm{~N}(\operatorname{det} \tau)^{-1}
$$

The identity in the lemma follows.

By the above lemmas, our global formula can be stated as follows:

$$
\begin{aligned}
\int_{H(k) \backslash H\left(\mathbb{A}_{k}\right)} E(h \tau, \mu, f) d h= & \left.\chi^{-1}\left(\mathrm{~N}_{F / k} \delta_{F}\right) \operatorname{vol}\left(\mathbb{A}_{k}^{\times} F^{\times} \backslash \mathbb{A}_{F}^{\times}\right)\right) L\left(0, \chi^{2}\right) L\left(1, \chi^{2}\right) . \\
& \cdot \chi\left(\delta_{F / k} / \delta_{D}\right) d_{F / k}^{-1 / 2} d_{D}^{1 / 2} \prod_{v \mid \delta_{D}}\left(1-\chi_{v}^{2}\left(\varpi_{v}\right)\right) .
\end{aligned}
$$

We may write every thing in terms of the representation $\sigma=\pi\left(\chi, \chi^{-1} \eta\right)$, using the following identities:

$$
\begin{aligned}
\operatorname{vol}\left(\mathbb{A}_{k}^{\times} F^{\times} \backslash \mathbb{A}_{F}^{\times}\right) & =2 d_{F}^{1 / 2} d_{k}^{-1 / 2} L(1, \eta), \\
L\left(0, \chi^{2}\right) & =\chi^{2}\left(\delta_{k}\right) d_{k}^{1 / 2} L\left(1, \chi^{-2}\right), \\
\mathrm{N}_{F / k} \delta_{F} & =\delta_{F / k} \delta_{k}^{2}, \\
\chi\left(\delta_{D}\right)^{-1} \prod_{v \mid \delta_{D}}\left(1-\chi_{v}\left(\varpi_{v}\right)\right) & =\prod_{v \mid \delta_{D}} \lambda_{v}^{*} .
\end{aligned}
$$

Proposition 3.2.5. Let $d_{k}$ and $d_{D}$ denote the absolute norms of the discriminants of $k$ and $D$ respectively. Then

$$
\int_{H(k) \backslash H\left(\mathbb{A}_{k}\right)} E(h \tau, \mu, f) d h=2 d_{k} d_{D}^{1 / 2} L\left(1, \operatorname{Sym}^{2} \sigma\right) \prod_{v \mid \delta_{D}}\left(\chi_{v}(\varpi)-\chi_{v}^{-1}(\varpi)\right)
$$

3.3. Volumes of Shimura subvarieties. In this section we compute $\operatorname{vol}\left(H(k) \backslash H\left(\mathbb{A}_{k}\right)\right)$ and deduce a formula from Proposition 3.2.5 for $\ell_{H}(\varphi)$ as in Theorem 1.2.1 when $\varphi$ is our standard Eisenstein series

$$
\varphi=E(g ; \mu, f) .
$$

We will actually use Proposition 3.2.5 and analyze poles of the Eisenstein series. First of all we need to compute the constant term of the Eisenstein series. 
Let $d x$ be the Haar measure on $\mathbb{A}_{F}$ such that together with the counting measure on $F$ it gives rise to the normalized measure on $F \backslash \mathbb{A}_{F}$ with volume 1 . Then the constant term of the Eisentsetin series is given by

$$
C(g ; \mu, f):=\int_{F \backslash \mathbb{A}_{F}} E(n(x) g ; \mu, f) d x=\int_{F \backslash \mathbb{A}_{F}} \sum_{\gamma \in B(F) \backslash \mathrm{GL}_{2}(F)} f(\gamma n(x) g) d x
$$

where $n(x)=\left(\begin{array}{ll}1 & x \\ 0 & 1\end{array}\right)$. Using decomposition

$$
\mathrm{GL}_{2}(F)=B(F) \coprod B(F) w N(F), \quad w=\left(\begin{array}{cc}
0 & 1 \\
-1 & 0
\end{array}\right)
$$

one obtains

$$
C(g ; \mu, f)=f(g)+\widetilde{f}(g), \quad \widetilde{f}(g)=\int_{\mathbb{A}_{F}} f(w n(x) g) d x .
$$

To compute $f$ we observe that $f$ is invariant under the right translation by $U$ and has character

$$
\mu_{B}:=\left(\mu|\cdot|^{1 / 2}, \mu^{-1}|\cdot|^{-1 / 2}\right)
$$

under the left translation of $B$. Thus, we need only to compute $f(e)$ :

$$
f(e)=\mu^{-1}\left(\delta_{F}\right) \int_{\mathbb{A}_{F}^{\times}} \phi((0, t)) \mu^{2}(t)|t|_{\mathbb{A}_{F}} d^{\times} t=\mu^{-1}\left(\delta_{F}\right) L\left(1, \mu^{2}\right) .
$$

Similarly, $\widetilde{f}_{\phi}$ is invariant from right by $U$ and has character

$$
\widetilde{\mu}_{B}=\left(\mu^{-1}|\cdot|^{-1 / 2}, \mu|\cdot|^{1 / 2}\right) .
$$

Thus, we also need only to compute $\widetilde{f}_{\phi}(e)$ :

$$
\begin{aligned}
\widetilde{f}(e) & =\int_{\mathbb{A}_{F}} f(w n(x)) d x=\mu^{-1}\left(\delta_{F}\right) \int_{\mathbb{A}_{F}} \int_{\mathbb{A}_{F}^{\times}} \phi((0, t) w n(x)) \mu^{2}(t)|t|_{\mathbb{A}_{F}} d^{\times} t d x \\
& =\mu^{-1}\left(\delta_{F}\right) \int_{\mathbb{A}_{F}} \int_{\mathbb{A}_{F}^{\times}} \phi(-t,-t x) \mu^{2}(t)|t|_{\mathbb{A}_{F}} d^{\times} t d x
\end{aligned}
$$

Using the change of variables $x \longrightarrow-x t^{-1}, t \longrightarrow-t$, we obtain

$$
\begin{aligned}
\widetilde{f}(e) & =\mu^{-1}\left(\delta_{F}\right) \int_{\mathbb{A}_{F}^{\times}} \int_{\mathbb{A}} \phi(t, x) \mu^{2}(t) d x d^{\times} t \\
& =\mu\left(\delta_{F}\right)^{-1} L\left(0, \mu^{2}\right)\left|\delta_{F}\right|^{1 / 2} .
\end{aligned}
$$

Note that the first pole of $\widetilde{f}$ from the right hand side is at $\mu=\|\cdot\|^{1 / 2}$ and has a constant residue. Thus we obtain the following: 
Lemma 3.3.1. The Eisensetin series $E(g ; \mu, f)$ for $\mu(x)=|x|^{s}$ has a pole at $s=1 / 2$ with residue given by

$$
\lim _{s \rightarrow 1 / 2}(s-1 / 2) \zeta_{F}(2 s)
$$

Now we take the residue at $s=\frac{1}{2}$ from both sides of the identity in Proposition 3.2.5 for $\mu(x)=|x|_{F}^{s}, \chi(x)=|x|_{k}^{s}$. By Lemma 3.3.1, we then obtain

$$
\operatorname{vol}\left(H(k) \backslash H\left(\mathbb{A}_{k}\right)\right)=d_{D}^{1 / 2} d_{k} \lim _{s \longrightarrow 1 / 2} \frac{L(1, \operatorname{Sym} \sigma)}{\zeta_{F}(2 s)} \prod_{v \mid \delta_{D}}\left(\left|\varpi_{v}\right|^{s}-\left|\varpi_{v}\right|^{-s}\right) .
$$

Recall

$$
\begin{aligned}
L\left(1, \operatorname{Sym}^{2} \sigma\right) & =L\left(1, \chi^{2}\right) L\left(1, \chi^{-2}\right) L(1, \eta) \\
& =\zeta_{k}(1+2 s) \zeta_{k}(1-2 s) L(1, \eta) \\
& =\zeta_{k}(1+2 s) d_{k}^{1 / 2} d_{k}^{2 s-1} \zeta_{k}(2 s) L(1, \eta)
\end{aligned}
$$

It follows that

Proposition 3.3.2. We have the explicit formula:

$$
\operatorname{vol}\left(H(k) \backslash H\left(\mathbb{A}_{k}\right)\right)=2 d_{D}^{1 / 2} d_{k}^{3 / 2} \zeta_{k}(2) \prod_{v \mid \delta_{D}}\left(\left|\varpi_{v}\right|^{1 / 2}-\left|\varpi_{v}\right|^{-1 / 2}\right) .
$$

Combined with Proposition 3.2.5, this proves the Theorem 1.2.1 in the case of Eisenstein series.

\section{Theta Lifting}

In this section, we will study periods of cusp forms using theta lifting. More precisely, we will identify $\operatorname{Res}_{F / k}\left(\mathrm{PGL}_{2}\right)$ (resp. $D^{\times} / k^{\times}$) as the adjoint group of $\operatorname{GSO}(V)$ (resp. the group $S O\left(V_{1}\right)$ ) with $V$ the space of hermitian matrices (resp. with $V_{1}$ a subspace of $V$ of codimension 1). Then the theta lifting gives a correspondence between forms on $\operatorname{GSO}\left(V_{\mathbb{A}_{k}}\right)$ and $\operatorname{GL}\left(2, \mathbb{A}_{k}\right)_{+}$, the subgroup of $\mathrm{GL}_{2}(2, \mathbb{A})$ with determinants in $\mathrm{N}_{F / k}\left(\mathbb{A}_{F}^{\times}\right)$. We will show that for a form $\varphi$ on $\operatorname{GSO}(V)$, the nonvanishing of the periods over $H$ implies that $\varphi$ is a theta lifting from $f$ on $\operatorname{GL}(2, \mathbb{A})_{+}$. Then we can use the Siegel-Weil formula to evaluate the periods and show that Theorem 1.2.1 is true up to certain universal constant at a finite number of bad places. All these results can be extended to Eisenstein series which will be used in the final determination of these universal constants. 
4.1. The Isogeny. We first describe a homomorphism from $\operatorname{Res}_{F / k}\left(\mathrm{GL}_{2}\right)$ to an orthogonal group attached to a quadratic form over $k$ in 4 variables.

Recall that $F / k$ is a quadratic extension of totally real fields. Denote by $x \longrightarrow \bar{x}$ the Galois involution of $F$ over $k$, and let $V$ be the space of $2 \times 2$ hermitian matrices with entries in $F$. This is a vector space over $k$ of dimension 4. We define a quadratic form $q$ on $V$ via $q(x)=\operatorname{det}(A)$. Note that

$$
\operatorname{det}(A)=\operatorname{det}\left(\begin{array}{ll}
a & b \\
b & d
\end{array}\right)=a d-b \bar{b} .
$$

Thus $q$ has signature $(2,2)$ over any real place of $k$, but its Witt index over $k$ is 1. The associated symmetric bilinear form is

$$
(A, B)=\operatorname{tr}\left(A B^{*}\right)
$$

where

$$
B^{*}=\operatorname{tr}(B) I-B
$$

We write $O(V)$ for the orthogonal group of $V, \mathrm{GO}(V)$ for the group of orthogonal similitudes, and $\operatorname{GSO}(V)$ and $\mathrm{SO}(V)$ the connected components of identity for $\mathrm{GO}(V)$ and $O(V)$ respectively.

Description of $\mathrm{GO}(V)$.

Proposition 4.1.1. Let $a \in k^{\times}$act on $V$ via scalar multiplication by a, and let $c$ act on $V$ by conjugation on $F / k$. Let $\gamma \in \mathrm{GL}_{2}(F)$ act on $V$ by

$$
\gamma(A)=\gamma A \bar{\gamma}^{t} \text {. }
$$

Then:

(1) $\mathrm{GO}(V)$ is generated by $\gamma \in \mathrm{GL}_{2}(F), a \in \mathrm{GL}_{1}(k)$, and $c$ with similitudes given by

respectively.

$$
\mathrm{N}_{F / k}(\operatorname{det} \gamma), \quad a^{2}, \quad 1
$$

(2) $\mathrm{GSO}(V)$ is generated by $\mathrm{GL}_{1}(k)$ and $\mathrm{GL}_{2}(F)$, and is equal to the quotient of $k^{\times} \times \mathrm{GL}_{2}(F)$ modulo the subgroup of elements $\left(\mathrm{N}(z)^{-1}, z I_{2}\right)$ for $z \in F^{\times}$.

(3) $\mathrm{SO}(V)$ is the subgroup of $\mathrm{GSO}(V)$ of elements $(\alpha, \gamma)$ such that

$$
\alpha^{2} \mathrm{~N}_{F / k}(\operatorname{det} \gamma)=1 \text {. }
$$

Proof. We need only prove (1). It follows from the definition that the described actions by $\mathrm{GL}_{2}(F), k^{\times}$, and $c$ are indeed in $G O(V)$. We want to show that every element $g \in G O(V)$ is a composition of these three kinds of operators.

The space $V$ has a decomposition $V=k e_{1}+k e_{2}+F e_{3}$ with

$$
e_{1}=\left(\begin{array}{ll}
1 & 0 \\
0 & 0
\end{array}\right), \quad e_{2}=\left(\begin{array}{ll}
0 & 0 \\
0 & 1
\end{array}\right), \quad e_{3}=\left(\begin{array}{ll}
0 & 1 \\
1 & 0
\end{array}\right) \text {. }
$$


We are done by performing the following steps:

(1) For any $v \in V$ such that $q(v)=0, v \neq 0$, there are $\gamma \in \mathrm{GL}_{2}(F), \lambda \in k^{\times}$ such that

$$
\gamma e_{1}=\lambda v
$$

Applying this to $v=g^{-1} e_{1}$ and replacing $g$ by $g \gamma$, we may assume that $g$ fixes the line $k e_{1}$. Moreover the stabilizer of $k e_{1}$ in $\mathrm{GL}_{2}(F)$ is the group $B(F)$ of upper triangular matrices.

(2) For any $v \in V$ such that $q(v)=0, v \neq 0, e_{1}$, there are $\gamma \in B(F), \lambda \in k^{\times}$ such that

$$
\gamma e_{2}=\lambda e_{2}
$$

Applying this to $v=g^{-1} e_{2}$, and replacing $g$ by $g \gamma$, we may assume $g$ fixes the lines $k e_{1}, k_{2} e_{2}$. Moreover the stabilizer of double lines $k e_{1}$ and $k e_{2}$ in $\mathrm{GL}_{2}(F)$ are the group $M(F)$ of diagonal matrices.

(3) For any $v \in F^{\times} e_{3}$, there is a $\gamma \in M(F)$ such that $\gamma v=e_{3}$. Applying this to $v=g^{-1} e_{3}$ we may assume that

$$
g e_{1}=\lambda e_{1}, \quad g e_{2}=\mu e_{3}, \quad g e_{3}=e_{3} .
$$

In this case $\lambda \mu=1$, and $g$ is product of a scalar multiplication of $\mu$, and a matrix action by

$$
\left(\begin{array}{ll}
\lambda & 0 \\
0 & 1
\end{array}\right)
$$

and a possible action by $c$.

Lemma 4.1.2. The group $\mathrm{SO}(V)$ is isomorphic to the group of elements $g \in$ $\mathrm{GL}_{2}(F)$ with determinant in $k$ modulo the scalars. Here $g$ acts on $V$ by

$$
g(v)=\operatorname{det}(g)^{-1} \cdot g \cdot v \cdot \bar{g}^{t} .
$$

Proof. First, the action given in the lemma defines a injective map

$$
\left\{g \in \mathrm{GL}_{2}(F): \quad \operatorname{det} g \in k\right\} / k^{\times} \longrightarrow \mathrm{SO}(V) .
$$

We need only show that this map is surjective. Let $h$ be an element in $\mathrm{SO}(V)$ represented by $(\alpha, g)$ with $\alpha \in k^{\times}, g \in \mathrm{GL}_{2}(F)$ such that

$$
\alpha^{2} \mathrm{~N}_{F / k}(\operatorname{det} g)=1 \text {. }
$$

Then $\mathrm{N}_{F / k}(\alpha \cdot \operatorname{det} g)=1$. Thus there is a $z \in F^{\times}$such that

$$
\alpha \cdot \operatorname{det} g=z / \bar{z} \text {. }
$$

Now we may replace $(\alpha, g)$ by $\left(\alpha z \bar{z}, \quad g \cdot z^{-1}\right)$ to assume that $\operatorname{det} g \in k^{\times}$. 
Image of $H=D^{\times} / k^{\times}$. Recall that we have fixed an embedding $\iota$ of $D$ into $M_{2}(F)$ by

$$
\iota(x+y \lambda)=\left(\begin{array}{ll}
x & y \epsilon \\
\bar{y} & \bar{x}
\end{array}\right) .
$$

One has the obvious formula

$$
\operatorname{det} \iota(h)=\mathrm{N}_{D}(h)
$$

where $\mathrm{N}_{D}(h)$ denote the norm map on $D$.

What is the action of $D^{\times}$on $V$ ? A simple calculation shows that $D^{\times}$fixes the line through

$$
e_{\epsilon}=\left(\begin{array}{cc}
\epsilon & 0 \\
0 & -1
\end{array}\right) \text {. }
$$

Moreover, one can show that the stabilizer of $e_{\epsilon}$ is simply $F^{\times} \cdot D^{\times}$. Thus with our modified action given in Lemma 4.1.2, we have that the stabilizer of $e_{\epsilon}$ in $\mathrm{SO}(V)$ is given by $D^{\times} / k^{\times}$. Conversely, any line in $V$ with norm $-\epsilon\left(k^{\times}\right)^{2}$ modulo $k^{\times}$is fixed by a conjugate of $D^{\times} / k^{\times}$.

Let $V_{1}$ be the orthogonal complement of $e_{\epsilon}$ :

$$
V_{1}=\left\{\left(\begin{array}{cc}
a \epsilon & b \\
\bar{b} & a
\end{array}\right): \quad a \in k, b \in F\right\}
$$

Let $D^{0}$ be the pure quaternions, namely the set of trace free elements in $D$. One checks that the restriction of $q$ to $V_{1}$ corresponds to the restriction of $\epsilon \cdot \xi^{2} \cdot \mathrm{N}_{D}$ on $D^{0}$ via the following isomorphism of $k$-vector spaces:

$$
V_{1} \longrightarrow D^{0}: \quad\left(\begin{array}{cc}
a \epsilon & b \\
\bar{b} & a
\end{array}\right) \mapsto \xi \cdot\left(\begin{array}{cc}
a & -b \\
\bar{b} \epsilon^{-1}-a
\end{array}\right)
$$

where $\xi$ is a nonzero trace free element in $F$. This map identifies the action of $D^{\times}$on $V_{1}$ with its action by $\mathrm{Ad}$ on $D^{0}$. In other words, as a representation of $D^{\times}$one has

$$
V \simeq \operatorname{trivial} \oplus \mathrm{Ad}
$$

Note that the image of $D^{\times}$under Ad is exactly $\mathrm{SO}\left(D^{0}\right)$.

4.2. Theta lifting. For the moment we consider algebraic groups over $k$. Set

$$
G L(2)_{/ F}=\operatorname{Res}_{F / k} G L(2)
$$

In Proposition 4.1.1, Part (2) of the last section, we have defined an algebraic homomorphism

$$
\nu: \quad G L(1) \times G L(2) / F \longrightarrow G S O(V),
$$

which is surjective and defined over $k$. Let $\pi$ be an irreducible automorphic representation of $G L(2)_{/ F}$, and let $\chi$ be an automorphic character of $G L(1)$. Suppose that the representation $\chi \otimes \pi$ of $G L(1) \times G L(2)_{/ F}$ is trivial on the kernel of the map $\nu$. Then the central character of $\pi$ must be $\chi \circ \mathrm{N}_{F / k}$. In such a 
case it defines a representation of $G S O(V)$ with central character $\chi$. Conversely any irreducible automorphic representation of $G S O(V)$ with a central character $\chi$ defines an automorphic representation $\chi \otimes \pi$ as above, where $\pi$ has central character $\chi \circ \mathrm{N}_{F / k}$. We note that once $\pi$ is given, the character $\chi$ is determined up to a twist by the $\eta_{F / k}$, the quadratic character associated to the extension $F / k$. The obvious local analogue of this discussion also applies.

Suppose we are in the global situation, with $\pi$ and $\chi$ as above. Let $V_{\pi}$ be the space of $\pi$. For any $\varphi \in V_{\pi}$ let $\varphi_{\chi}$ be the function on $\operatorname{GL}\left(1, \mathbb{A}_{k}\right) \times \operatorname{GL}\left(2, \mathbb{A}_{F}\right)$ defined by

$$
\varphi_{\chi}(t, x)=\chi(t) \varphi(x)
$$

Define a function $\tilde{\varphi}_{\chi}$ on $\operatorname{GSO}(V)$ by the formula

$$
\tilde{\varphi}_{\chi}(\nu(t, x))=\varphi_{\chi}(t, x) .
$$

It is easy to see that $\tilde{\varphi}_{\chi}$ is an automorphic form on $\operatorname{GSO}(V)$. Similarly, we can push the representation $\chi \otimes \pi$ forward to $\operatorname{GSO}(V)$; call it $\pi_{\chi}$. By this kind of correspondence, we see in particular that automorphic forms on $\operatorname{GSO}(V)$ have the strong multiplicity one property.

For the rest of this section we assume that $\pi$ has trivial central character and actually that $\chi=1$. We write

$$
G=\mathrm{GSO}(V), \quad G_{1}=\mathrm{SO}(V), \quad H=\mathrm{SO}\left(V_{1}\right)
$$

where $V_{1}$ is the orthogonal complement of a line fixed by $H$ with nonzero norms. If $\varphi$ is an automorphic function on $G$ we define its period relative to $H$ by the formula

$$
\ell_{H}(\varphi)=\int_{H(k) \backslash H\left(\mathbb{A}_{k}\right)} \varphi(x) d x=\int_{\mathrm{SO}\left(V_{1}\right)_{k} \backslash \mathrm{SO}\left(V_{1}\right)_{\mathbb{A}_{k}}} \varphi(x) d x .
$$

Of course this period integral also makes sense if $\varphi$ is just an automorphic function on $G_{1}$.

We shall relate the non-vanishing of period integrals to the existence of a certain theta lift. Fix an additive character $\psi$ for $\mathbb{A}_{k} / k$. Then the Weil representation $\omega$ of $G_{1} \times \mathrm{SL}_{2}$ can be realized on $\mathcal{S}\left(V_{\mathbb{A}_{k}}\right)$, the space of Bruhat-Schwartz functions on $V_{\mathbb{A}_{k}}$, with (part of the) actions given by

$$
\left\{\begin{array}{l}
\omega\left(g_{1}\right) \phi(v)=\phi\left(g_{1}^{-1} v\right), \\
\omega\left(\left(\begin{array}{ll}
1 & x \\
0 & 1
\end{array}\right)\right) \phi(v)=\psi(x q(v)) \phi(v) .
\end{array}\right.
$$

We would like to extend this to similitude groups, following Jacquet-Langlands [11] and Harris-Kudla [9]. In what follows all facts stated about theta correspondence for similitude groups can be found in [9], unless otherwise specified. (Note however that our $G$ is denoted $H$ in [9], and they use $\mathrm{GO}(V)$ instead of $\operatorname{GSO}(V)$ ). 
First we define an action of $\mathrm{GO}(V)$ on $\mathcal{S}\left(V_{\mathbb{A}_{k}}\right)$. For $g \in \mathrm{GO}(V)$ let $\lambda(g)$ be the similitude factor of $g$. We let $L(g)$ be the unitary operator defined by

$$
L(g) \phi(v)=|\lambda(g)|^{-1} \phi\left(g^{-1} v\right) .
$$

We may view GL(2) over $k$ as the similitude group of a two dimensional symplectic form. The similitude factor of $h \in \mathrm{GL}(2)$ is then just det $h$. Let $R$ be the closed subgroup of $G \times \mathrm{GL}(2)$ defined by

$$
R=\{(g, h) \in G \times \mathrm{GL}(2) \mid \lambda(g)=\operatorname{det} h\} .
$$

If $A$ is an algebra over $k$, then

$$
R(A)=\{(g, h) \in G(A) \times \mathrm{GL}(2, A) \mid \lambda(g)=\operatorname{det} h\} .
$$

Now we define

$$
\omega(g, h) \phi(v)=L(g) \omega\left(h_{1}\right) \phi(v),
$$

where

$$
h=\left(\begin{array}{cc}
1 & 0 \\
0 & \operatorname{det} h
\end{array}\right) h_{1} .
$$

One checks that this defines a representation of $R\left(\mathbb{A}_{k}\right)$ on $\mathcal{S}\left(V_{\mathbb{A}_{k}}\right)$, extending that of $G_{1}\left(\mathbb{A}_{k}\right) \times \mathrm{SL}\left(2, \mathbb{A}_{k}\right)$ already defined above. As usual, one has $\omega=\otimes \omega_{v}$, with $\omega_{v}$ defined by formulas analogous to the ones given above. For each $\phi \in \mathcal{S}\left(V_{\mathbb{A}_{k}}\right)$, we define a theta function on $R\left(\mathbb{A}_{k}\right)$ by the usual formula

$$
\theta(g, h ; \phi)=\sum_{\xi \in V(k)} \omega(g, h) \phi(\xi) .
$$

From [9] we know that $\theta(g, h ; \phi)$ is left $R(k)$-invariant as a function on $R\left(\mathbb{A}_{k}\right)$.

Let $\mathrm{GL}(2)+$ be the subgroup of $\mathrm{GL}(2)$ defined by

$$
\mathrm{GL}(2)_{+}=\left\{h \in \mathrm{GL}(2) \mid \operatorname{det} h \in \lambda(G)=\mathrm{N}_{F / k}\left(F^{\times}\right)\right\} .
$$

Let $v$ be a place of $k$. If $v$ splits in $F$ then $\operatorname{GL}\left(2, k_{v}\right)_{+}=\operatorname{GL}\left(2, k_{v}\right)$. Otherwise, $\mathrm{GL}\left(2, k_{v}\right)_{+}$is a subgroup of index 2 in $\operatorname{GL}\left(2, k_{v}\right)$. We also note that $\operatorname{GL}\left(2, \mathcal{O}_{v}\right) \subset$ $\mathrm{GL}\left(2, k_{v}\right)_{+}$whenever $v$ is a finite place unramified in $F$. Let $\pi_{v}, \sigma_{v}$ be irreducible admissible representations of $G\left(k_{v}\right)$ and $\mathrm{GL}\left(2, k_{v}\right)_{+}$, respectively. We say that $\pi_{v}$ and $\sigma_{v}$ are in theta correspondence with each other if

$$
\operatorname{Hom}_{R\left(k_{v}\right)}\left(\omega_{v}, \pi_{v} \otimes \sigma_{v}\right) \neq 0
$$

Based on work of Waldspurger [20], Brooks Roberts [18] has shown that whenever $v$ is finite with odd residue characteristic, theta correspondence defines a bijection of certain subsets of the admissible duals of $G\left(k_{v}\right)$ and $\mathrm{GL}\left(2, k_{v}\right)_{+}$. (Of course, Roberts' result is more general, covering arbitrary symplectic-orthogonal dual pairs). In other words, Howe's local duality conjecture is valid in the present setting. 
But the theta correspondence of [18] is phrased in terms of the pair $\operatorname{GO}(V)$ and $\mathrm{GL}(2)_{+}$. So we need to explain why it suffices to consider $\operatorname{GSO}(V)$ and $\mathrm{GL}(2)_{+}$ here. Recall from the last section that $V$ is the orthogonal direct sum of $-F$ and a hyperbolic plane. Let $c$ be the non-trivial Galois involution of $F / k$, which is extended to a reflection on $V$ by making it trivial on the hyperbolic plane. Then $\mathrm{GO}(V)$ is the semi-direct product of $G S O(V)$ and the two-element group $\{1, c\}$. (See Proposition 4.1.1)

Fix a place $v$ and let $\pi_{v}$ be an irreducible representation of $\operatorname{GSO}(V)_{v}=G\left(k_{v}\right)$. Then we may twist $\pi_{v}$ by $c$ and get a new representation $\pi_{v}^{c}$. (This is the composition of $\pi_{v}$ with conjugation by $c$ ). There are two possibilities:

(1). $\pi_{v}^{c} \simeq \pi_{v}$. In this case there are two possible extensions of $\pi_{v}$ to $\operatorname{GO}(V)_{v}$. Let $\tilde{\pi}_{v}$ be one such extension. Let sgn be the unique non-trivial character of $\mathrm{GO}(V)_{v}$ which is trivial on $\operatorname{GSO}(V)_{v}$. Then the other extension of $\pi_{v}$ is just the tensor product of $\tilde{\pi}_{v}$ with sgn. A well known argument (see below) shows that at most one of these two representations of $\mathrm{GO}(V)_{v}$ could occur in theta correspondence with $\mathrm{GL}\left(2, k_{v}\right)_{+}$.

(2). $\pi_{v}^{c} \nsucceq \pi_{v}$. In this case $\pi_{v}$ induces to an irreducible representation of $\mathrm{GO}(V)_{v}$.

It is well known (Mackey theory) that all irreducible representations of $\mathrm{GO}(V)_{v}$ are obtained in the manner described in (1) and (2) above.

We claim that, in the context of theta correspondence with $\mathrm{GL}(2)_{+}$, only possibility (1) could arise. Suppose we are in the situation of (2). Let $\tilde{\pi}_{v}$ be the representation of $\mathrm{GO}(V)_{v}$ induced from $\pi_{v}$, and suppose that it occurs in the theta correspondence. Clearly we have $\tilde{\pi}_{v} \simeq \operatorname{sgn} \otimes \tilde{\pi}_{v}$, and so the later also occurs in the theta correspondence. This implies that $\operatorname{sgn} \otimes \tilde{\pi}_{v} \otimes \tilde{\pi}_{v}^{*}$ occurs as a quotient of a Weil representation associated $\mathrm{GSp}_{4}$. That is, $\operatorname{sgn} \otimes \tilde{\pi}_{v} \otimes \tilde{\pi}_{v}^{*}$ is a quotient of $\omega_{v} \otimes \omega_{v}^{*}$ which can be viewed as the restriction to $\operatorname{GO}(V)_{v}$ of a Weil representation associated to the pair $\mathrm{GO}(V)$ and $\mathrm{GSp}_{4}$. Since sgn is obviously a quotient of $\operatorname{sgn} \otimes \tilde{\pi}_{v} \otimes \tilde{\pi}_{v}^{*}$, we see that sgn occurs in theta correspondence with $\mathrm{GSp}_{4}$. By restriction, we see that the determinant character of $\mathrm{O}(V)$ occurs in the "classical" theta correspondence associated with the reductive dual pair $\left(\mathrm{O}(V), \mathrm{Sp}_{4}\right)$. This contradicts the known fact that $\operatorname{det}(\cdot)$ of an orthogonal group $\mathrm{O}(V)$ does not occur in the theta correspondence with $\operatorname{Sp}_{2 n}$ unless $\operatorname{dim} V \leq n$. This proves our claim.

For any cuspidal automorphic function $\varphi$ on $G\left(\mathbb{A}_{k}\right)$ and any $\phi \in \mathcal{S}\left(V_{\mathbb{A}_{k}}\right)$ we now define a function on $\operatorname{GL}\left(2, \mathbb{A}_{k}\right)_{+}$by the formula

$$
\theta_{\phi}^{\varphi}(h)=\theta(\varphi ; \phi)(h)=\int_{G_{1}(k) \backslash G_{1}\left(\mathbb{A}_{k}\right)} \theta\left(g_{1} g, h ; \phi\right) \varphi\left(g_{1} g\right) d g_{1}
$$


where $g$ is any element in $G\left(\mathbb{A}_{k}\right)$ with $\lambda(g)=\operatorname{det} h$ (the result being independent of this choice). Then $\theta_{\phi}^{\varphi}$ is left invariant under $\operatorname{GL}(2, k)_{+}$. The definition of $\theta_{\phi}^{\varphi}(h)$ is compatible with theta correspondence in the sense that

$$
\theta(\varphi ; \omega(g, h) \phi)(x)=\theta\left(r\left(g^{-1}\right) \varphi ; \phi\right)(x h)
$$

for any $(g, h) \in R\left(\mathbb{A}_{k}\right)$ and $x \in \operatorname{GL}\left(2, \mathbb{A}_{k}\right)_{+}$. Here $r$ denotes the usual action by right translations. This was given in Harris-Kudla [9] for the case $(g, h) \in$ $\mathrm{SO}(V)_{\mathbb{A}_{k}} \times \mathrm{SL}\left(2, \mathbb{A}_{k}\right)$ but its extension to all of $R(\mathbb{A})$ is obvious.

The unipotent subgroups of GL(2)+ are the same as those of GL(2). Thus the notion of cuspidality for functions on $\mathrm{GL}(2, k)_{+} \backslash \mathrm{GL}\left(2, \mathbb{A}_{k}\right)_{+}$can be defined in the obvious way. Any function $f$ on $\mathrm{GL}(2, k)_{+} \backslash \mathrm{GL}\left(2, \mathbb{A}_{k}\right)_{+}$extends to a unique function $\tilde{f}$ on $\operatorname{GL}(2, k) \backslash \mathrm{GL}\left(2, \mathbb{A}_{k}\right)$ which is supported on $\operatorname{GL}(2, k) \cdot \operatorname{GL}\left(2, \mathbb{A}_{k}\right)_{+}$. It is easy to see that $\tilde{f}$ is cuspidal if and only if $f$ is cuspidal.

Proposition 4.2.1. Let $\varphi$ be a cuspidal automorphic function on $G\left(\mathbb{A}_{k}\right)$. If $\ell_{H}(\varphi) \neq 0$, then $\varphi$ has a nonzero cuspidal theta lift to $\mathrm{GL}\left(2, \mathbb{A}_{k}\right)_{+}$. In other words, there is a $\phi \in \mathcal{S}\left(V_{\mathbb{A}_{k}}\right)$ such that $f:=\theta_{\phi}^{\varphi} \neq 0$. Furthermore, the function $f$ is cuspidal.

Proof. Let $n(x)=\left(\begin{array}{ll}1 & x \\ 0 & 1\end{array}\right)$. For $t \in k$, define the $t$-th Fourier coefficient $f_{t}$ by

$$
f_{t}(h)=\int_{k \backslash \mathbb{A}_{k}} f(n(x) h) \psi(-t x) d x .
$$

From (4.2.2), we get

$$
f(n(x))=\int_{G_{1}(k) \backslash G_{1}\left(\mathbb{A}_{k}\right)} \sum_{\xi \in V(k)} \psi(x q(\xi)) \phi\left(g^{-1} \xi\right) \varphi(g) d g .
$$

Hence

$$
f_{t}(1)=\int_{G_{1}(k) \backslash G_{1}\left(\mathbb{A}_{k}\right)} \sum_{q(\xi)=t} \phi\left(g^{-1} \xi\right) \varphi(g) d g .
$$

First suppose $t \neq 0$. By Witt's theorem the set $\{\xi \mid q(\xi)=t\}$ is a single orbit under $G_{1}(k)$ (or empty). Take a $\xi \in V(k)$ such that $q(\xi)=t$ (if no such $\xi$ exists then $\left.f_{t}(1)=0\right)$. Let $G_{\xi}$ be the stabilizer of $\xi$ in $G_{1}$. Then

$$
\begin{aligned}
f_{t}(1) & =\int_{G_{1}(k) \backslash G_{1}\left(\mathbb{A}_{k}\right)} \sum_{\gamma \in G_{\xi}(k) \backslash G_{1}(k)} \phi\left(g^{-1} \gamma^{-1} \xi\right) \varphi(g) d g \\
& =\int_{G_{\xi}(k) \backslash G_{1}\left(\mathbb{A}_{k}\right)} \phi\left(g^{-1} \xi\right) \varphi(g) d g \\
& =\int_{G_{\xi}\left(\mathbb{A}_{k}\right) \backslash G_{1}\left(\mathbb{A}_{k}\right)}\left(\int_{G_{\xi}(k) \backslash G_{\xi}\left(\mathbb{A}_{k}\right)} \varphi(h g) d h\right) \phi\left(g^{-1} \xi\right) d g .
\end{aligned}
$$


Apply this to a nonzero vector $\xi_{0}$ in $V_{1}^{\perp}$. Then $t_{0}=q\left(\xi_{0}\right) \neq 0, H=G_{\xi_{0}}$. If $\ell_{H}(\varphi) \neq 0$, we see from the above formula that $f_{t_{0}}(1) \neq 0$ for some choice of $\phi$.

Now consider $f_{0}$. The set $\{\xi: q(\xi)=0\}$ breaks up into two orbits, namely $\{0\}$ and $\{\xi \neq 0: \quad q(\xi)=0\}$. We get

$$
f_{0}(1)=\int \phi(0) \varphi(g) d g+\int \sum_{q(\xi)=0, \xi \neq 0} \phi\left(g^{-1} \xi\right) \varphi(g) d g .
$$

The first term is always zero since $\varphi$ is cuspidal. Fix $\xi_{0} \neq 0$ with $q\left(\xi_{0}\right)=0$. Let $P$ be the parabolic subgroup of $G_{1}$ stabilizing the line through $\xi_{0}$. Let $\xi_{1} \in V(k)$ be another isotropic vector with $\left(\xi_{0}, \xi_{1}\right)=1$. Let $V_{0} \subset V$ be the orthogonal complement of the hyperbolic plane spanned by $\xi_{0}, \xi_{1}$. Then $P=\operatorname{GL}(1) \operatorname{SO}\left(V_{0}\right) N$. The unipotent radical $N$ is nontrivial. One has $G_{\xi_{0}}=\mathrm{SO}\left(V_{0}\right) N$. Thus

$$
f_{0}(1)=\int_{G_{\xi_{0}}\left(\mathbb{A}_{k}\right) \backslash G_{1}\left(\mathbb{A}_{k}\right)}\left(\int_{G_{\xi_{0}}(k) \backslash G_{\xi_{0}}\left(\mathbb{A}_{k}\right)} \varphi(h g) d h\right) \phi\left(g^{-1} \xi\right) d g
$$

and the inner integral is

$$
\int_{G_{\xi_{0}}(k) \backslash G_{\xi_{0}}\left(\mathbb{A}_{k}\right)} \varphi(h g) d h=\int_{\mathrm{SO}\left(V_{0}\right)(k) \backslash \mathrm{SO}\left(V_{0}(\mathbb{A})\right)} \int_{N(k) \backslash N(\mathbb{A})} \varphi(n a g) d n d a=0,
$$

since $\varphi$ is cuspidal. Thus we see that the theta lift of $\varphi$ is always cuspidal.

Let $\pi=\otimes \pi_{v}$ be an irreducible cuspidal automorphic representation of $G\left(\mathbb{A}_{k}\right)$. We denote by $\Theta(\pi)$ the space of functions on $\operatorname{GL}\left(2, \mathbb{A}_{k}\right)+$ generated by

$$
\theta_{\phi}^{\varphi}, \quad\left(\phi \in \mathcal{S}\left(V\left(\mathbb{A}_{k}\right)\right), \varphi \in V_{\pi}\right)
$$

This is a space of cusp forms on $\operatorname{GL}(2, k)_{+} \backslash \mathrm{GL}\left(2, \mathbb{A}_{k}\right)_{+}$. Suppose it is non-zero. Let $V_{\sigma}$ be an irreducible summand of this space, corresponding to an irreducible cuspidal automorphic representation $\sigma=\otimes \sigma_{v}$ of $\mathrm{GL}(2)_{+}$. Then each $\sigma_{v}$ is a local theta lift of $\pi_{v}^{*}$, or equivalently, $\sigma_{v}^{*}$ is a local theta lift of $\pi_{v}$. Here $\pi_{v}^{*}$ denotes the contragredient of $\pi_{v}$, and similarly for $\sigma_{v}^{*}$. By the validity of the Howe duality conjecture over local fields with odd residue characteristic [18], we see that for almost all place $v$, the isomorphism class of $\sigma_{v}$ is uniquely determined by $\pi_{v}$. Thus the space $\Theta(\pi)$ is a finite sum of irreducible cuspidal automorphic representations of $G L(2)_{+}$.

We now try to lift $\sigma$ back to $G\left(\mathbb{A}_{k}\right)$. Define

$$
\theta_{\phi}^{f}(g)=\theta_{V}(f ; \phi)(g)=\int_{\mathrm{SL}(2, k) \backslash \mathrm{SL}\left(2, \mathbb{A}_{k}\right)} \theta\left(g, h_{1} h ; \phi\right) f\left(h_{1} h\right) d h_{1}
$$

for $\phi \in \mathcal{S}\left(V\left(\mathbb{A}_{k}\right)\right), f \in V_{\sigma}, g \in G\left(\mathbb{A}_{k}\right)$, where $h$ is any element of $\mathrm{GL}\left(2, \mathbb{A}_{k}\right)_{+}$such that $\operatorname{det} h=\lambda(g)$. Let $\Theta_{V}(\sigma)$ be the linear span of all such $\theta_{\phi}^{f}$. For any $\varphi \in V_{\pi}$ 
we have

$$
\begin{aligned}
<\theta_{\phi}^{f}, \bar{\varphi}>_{G_{1}} & =\iint \theta_{\phi}(g, h) f(h) \cdot \varphi(g) d h d g \\
& =\int\left(\int \theta_{\phi}(g, h) \varphi(g) d g\right) f(h) d h \\
& =<\theta_{\phi}^{\varphi}, \bar{f}>_{\mathrm{SL}(2)}
\end{aligned}
$$

It follows from this formula and the definitions that the space $\Theta_{V}(\sigma)$ is non-zero. By Lemma 5.1.9 of [9] (and the analogous result for theta lifting in the "other" direction), $\Theta_{V}(\sigma)$ has the same central character as $\pi$, say $\chi$.

Lemma 4.2.2. The space $\Theta(\sigma)$ consists of cusp forms.

Proof. Suppose not. A well known principle says that $\sigma$ is generated by binary theta series. In other words, $\sigma$ is a theta lift from $\operatorname{GSO}\left(F, \mathbb{A}_{k}\right)$, where $F$ is viewed as a two dimensional space over $k$ with quadratic form given by the norm form.

Since $\operatorname{GSO}\left(F, \mathbb{A}_{k}\right)=\mathbb{A}_{F}^{\times}$, this means that $\sigma$ is a cusp form attached to a grossencharacter $\chi$ of $\mathbb{A}_{F}^{\times}$by Jacquet and Langlands [11]. The restriction of $\chi$ to $\mathbb{A}_{k}^{\times}$must be equal to $\eta_{F / k}$, the quadratic character associated to the extension $F / k$. Note that in this case $\sigma$ induces to an irreducible cuspidal automorphic representation of GL(2) (see [11], Theorem 4.6). Now another familiar principle in the theory of theta correspondence (the induction principle) says that the local theta lift $\theta_{v}\left(\sigma_{v}^{*}\right)$ is a subquotient of an induced representation. More precisely, this induced representation is the local component of the representation of $G\left(\mathbb{A}_{k}\right)$ induced from the character

$$
\left(\begin{array}{ccc}
a N(u) & 0 & 0 \\
0 & r(u) & 0 \\
0 & 0 & a^{-1}
\end{array}\right) \mapsto \eta(u), \quad\left(u \in \mathbb{A}_{F}^{\times}, a \in \mathbb{A}_{k}^{\times}\right) .
$$

Since this is a unitary character and $G$ is a quotient of $\mathrm{GL}(1) \times \mathrm{GL}(2) / F$, we see that this induced representation is actually irreducible at each $v$, and is isomorphic to $\theta_{v}\left(\sigma_{v}^{*}\right) \simeq \pi_{v}$.

As already indicated, our representation $\pi$ corresponds to the automorphic cuspidal representation $\chi \otimes \tilde{\pi}$ of $G L(2)_{/ F}$. Making the map $\mathrm{GL}(1) \times \mathrm{GL}(2)_{/ F} \longrightarrow$ $G$ explicit, we see that the representation $\tilde{\pi}$ is (fully) induced from the character

$$
\left(\begin{array}{cc}
a_{1} & 0 \\
0 & a_{2}
\end{array}\right) \mapsto \eta\left(a_{1} \bar{a}_{2}\right), \quad\left(a_{1}, a_{2} \in \mathbb{A}_{F}^{\times}\right) .
$$

Let $L^{S}(s, \tilde{\pi})$ be the partial standard $L$-function attached to $\tilde{\pi}$. One has

$$
L^{S}(s, \tilde{\pi})=L^{S}(s, \eta) L^{S}(s, \bar{\eta})
$$

where $\bar{\eta}(a)=\eta(\bar{a})$. Thus $L^{S}\left(s, \eta^{-1} \otimes \tilde{\pi}\right)$ has a pole at $s=1$, which contradicts the fact that $\pi$, and therefore $\tilde{\pi}$, is cuspidal. 
Now let $\pi^{\prime}=\otimes \pi_{v}^{\prime}$ be any irreducible summand of $\Theta_{V}(\sigma)$. Then each $\pi_{v}^{\prime}$ is in theta correspondence with $\sigma_{v}^{*}$. By the results of Roberts [18] quoted earlier, we know that $\pi_{v}^{\prime} \simeq \pi_{v}$ for almost all $v$. By the strong multiplicity one property, we see that $\Theta_{V}(\sigma)$ is irreducible and coincides with (the space of) $\pi$.

The analogue of (4.2.5) is

$$
\theta_{V}(f ; \omega(g, h) \phi)=\theta_{V}\left(r\left(h^{-1}\right) f ; \phi\right)(x g), \quad\left((g, h) \in R\left(\mathbb{A}_{k}\right)\right)
$$

Now for any fixed $\phi$, the map

$$
f \mapsto \theta_{V}(f, \phi)
$$

is a homomorphism from $V_{\sigma}$ to $V_{\pi}$. On the space $\operatorname{Hom}\left(V_{\sigma}, V_{\pi}\right)$ we have the action of $R\left(\mathbb{A}_{k}\right)$ given by

$$
(g, h) \cdot T=\pi(g) T \sigma\left(h^{-1}\right)
$$

Then the relation (4.2.8) just says that

$$
\phi \mapsto \theta_{V}(\cdot ; \phi)
$$

is an $R\left(\mathbb{A}_{k}\right)$-intertwinning map from $\mathcal{S}\left(V_{\mathbb{A}_{k}}\right)$ to $\operatorname{Hom}\left(V_{\sigma}, V_{\pi}\right)$.

Let $K_{\mathbb{A}_{k}}$ and $K_{\mathbb{A}_{k}}^{\prime}$ be maximal compact subgroups of $G\left(\mathbb{A}_{k}\right)$ and $G L\left(2, \mathbb{A}_{k}\right)_{+}$ respectively. (Keep in mind that $G L\left(2, \mathcal{O}_{v}\right) \subseteq G L\left(2, k_{v}\right)_{+}$for almost all $v$ ). Set

$$
K_{R}=R\left(\mathbb{A}_{k}\right) \cap\left(K_{\mathbb{A}_{k}} \times K_{\mathbb{A}_{k}}^{\prime}\right),
$$

and let $\operatorname{Hom}\left(V_{\sigma}, V_{\pi}\right)_{K_{R}}$ be the space of $K_{R}$-finite vectors in $\operatorname{Hom}\left(V_{\sigma}, V_{\pi}\right)$. Then

$$
\operatorname{Hom}\left(V_{\sigma}, V_{\pi}\right)_{K_{R}} \simeq\left(V_{\pi}\right)_{K_{\mathbb{A}_{k}}} \otimes\left(V_{\sigma}^{*}\right)_{K_{\mathbb{A}_{k}}^{\prime}}
$$

The right hand side is algebraically irreducible. As usual, for an archimedean place $v$ we replace the action of the group by the joint action of the universal enveloping algebra and the maximal compact subgroup, i.e. the Harish-Chandra module structure.

Thus the map

$$
\mathcal{S}\left(V\left(\mathbb{A}_{k}\right)\right)_{K_{R}} \longrightarrow \operatorname{Hom}\left(V_{\sigma}, V_{\pi}\right)_{K_{R}}, \quad \phi \mapsto \theta_{V}(\cdot ; \phi)
$$

must be surjective. In particular, if $\varphi \in\left(V_{\pi}\right)_{K_{\mathrm{A}_{k}}}$ and $\bar{f} \in\left(V_{\sigma}^{*}\right)_{K_{\mathrm{A}_{k}}^{\prime}}$ then there is Bruhat-Schwartz function $\phi$ which is $K_{R}$-finite and such that

$$
\theta_{V}(\cdot ; \phi)=\varphi \otimes \bar{f}
$$

Here we use $\bar{f}$ to denote an element of $V_{\sigma}^{*}$ because when $V_{\sigma}$ is realized as a space of cusp forms, $V_{\sigma}^{*}$ is identified with the space of functions which are complex conjugates of functions in $V_{\sigma}$. This means

$$
\theta_{V}\left(f_{1} ; \phi\right)=<f_{1}, f>\cdot \varphi, \quad\left(f_{1} \in V_{\sigma}\right)
$$

In particular, we have

$$
\varphi=\frac{1}{|f|^{2}} \theta_{V}(f ; \phi) .
$$


If both $\varphi$ and $f$ are factorizable then $\phi$ can be chosen to be factorizable.

4.3. The Siegel-Weil formula. Let $\pi=\otimes \pi_{v}$ be a cuspidal automorphic representation of $G\left(\mathbb{A}_{k}\right)$, realized on the space $V_{\pi} \subset C^{\infty}\left(G(k) \backslash G\left(\mathbb{A}_{k}\right)\right.$. Suppose $\pi$ is a theta lift of a cusp form on $\mathrm{GL}\left(2, \mathbb{A}_{k}\right)_{+}$. For $\phi \in \mathcal{S}\left(V\left(\mathbb{A}_{k}\right)\right)$, we may define $\theta(g, x)$ as before, where $(g, x) \in R\left(\mathbb{A}_{k}\right)$. Our assumption says there is a cuspidal automorphic representation $\sigma$ of $\mathrm{GL}\left(2, \mathbb{A}_{k}\right)_{+}$on $V_{\sigma} \subset C^{\infty}\left(\mathrm{GL}(2, k)_{+} \backslash \mathrm{GL}\left(2, \mathbb{A}_{k}\right)_{+}\right)$, such that $V_{\pi}$ is generated by the integrals

$$
\varphi(g)=\int_{\mathrm{SL}(2, k) \backslash \mathrm{SL}\left(2, \mathbb{A}_{k}\right)} \theta\left(g, x x_{0}\right) f\left(x x_{0}\right) d x, \quad\left(\phi \in V\left(\mathbb{A}_{k}\right), f \in V_{\sigma}\right)
$$

where $x_{0}$ is any element in $\mathrm{GL}_{2}\left(\mathbb{A}_{k}\right)$ with determinant equal to $\lambda(g)$. For these forms, the periods becomes

$$
\int_{H(k) \backslash H\left(\mathbb{A}_{k}\right)} \varphi(h) d h=\int_{\mathrm{SL}(2, k) \backslash \mathrm{SL}\left(2, \mathbb{A}_{k}\right)} f(x) \int_{H(k) \backslash H\left(\mathbb{A}_{k}\right)} \theta(h, x) d h d x .
$$

Note that the inner integral is absolutely convergent if $H$ is non-split. Otherwise, we need to regularize the integral.

Let $\widetilde{\mathrm{SL}_{2}}(\mathbb{A})$ be the metaplectic two fold cover $\mathrm{SL}_{2}(\mathbb{A})$. We denote the covering map by $\widetilde{x} \longrightarrow x$. For any subset $X \subset \mathrm{SL}_{2}(\mathbb{A})$, let $\widetilde{X}$ denote its preimage in $\widetilde{\mathrm{SL}}_{2}(\mathbb{A})$.

We write $V=V_{0} \oplus V_{1}$. Then

$$
\mathrm{SO}\left(V_{0}\right) \times \mathrm{SO}\left(V_{1}\right)=\mathrm{SO}\left(V_{0}\right) \times H \subset \mathrm{SO}(V)=G_{1} .
$$

Consider the see-saw pairs

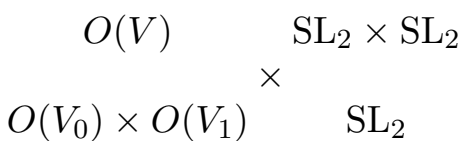

We let $\omega_{j}$ denote the Weil representation associated to the pair $\left(O\left(V_{j}\right), \widetilde{\mathrm{SL}}_{2}\right)$ realized on $\mathcal{S}\left(V_{j}\left(\mathbb{A}_{k}\right)\right)(j=0,1)$. For the sake of clarity, we assume that $\phi=$ $\phi_{0} \otimes \phi_{1}$ with $\phi_{j} \in \mathcal{S}\left(V_{j}(\mathbb{A})\right)$ and let $\theta_{j}$ be the corresponding theta functions. Then $\theta=\theta_{0} \cdot \theta_{1}$ and we have

$$
\int_{H(k) \backslash H\left(\mathbb{A}_{k}\right)} \theta(h, x) d h=\theta_{0}(1, \widetilde{x}) \int_{H(k) \backslash H\left(\mathbb{A}_{k}\right)} \theta_{1}(h, \widetilde{x}) d h .
$$

This integral can be evaluated via the Siegel-Weil formula:

$$
\int_{H(k) \backslash H\left(\mathbb{A}_{k}\right)} \theta_{1}(h, x) d h=E\left(x, \phi_{1}, 1 / 2\right)
$$


where $E\left(x, \phi_{1}, 1 / 2\right)$ is the Eisenstein series defined as follows. Let $P=M N$ be the upper triangular parabolic subgroup of $\mathrm{SL}(2)_{k}$. As usual, for $W$ an orthogonal space of dimension $n, M=\mathrm{GL}_{1}$ and $\widetilde{M}$ acts on $\mathcal{S}\left(W\left(\mathbb{A}_{k}\right)\right)$ by

$$
\omega_{V}(\widetilde{m}) \phi(w)=\gamma(\widetilde{m})^{n}|m|^{n / 2} \phi(w m) .
$$

Here $\gamma$ is a character on $\widetilde{M}$ satisfying

$$
\gamma(\widetilde{m})^{2}=(-1, m)_{k}
$$

where $(\cdot, \cdot)_{k}$ is the product of local Hilbert symbols $(\cdot, \cdot)_{v}$. When $n$ is even, $\omega_{V}$ can be viewed as a representation of $\mathrm{SL}_{2}$ with (4.3.6) replaced by

$$
\omega_{V}(m) \phi(w)=\eta_{V}(m)|m|^{n / 2} \phi(w m)
$$

where

$$
\eta_{V}(m)=(\operatorname{disc}(V), m)_{k} .
$$

Let $U$ be the standard maximal compact subgroup of $\mathrm{SL}_{2}\left(\mathbb{A}_{k}\right)$. Write any $x \in \mathrm{SL}_{2}\left(\mathbb{A}_{k}\right)$ as $x=n m u$ with $n \in N\left(\mathbb{A}_{k}\right), m \in M\left(\mathbb{A}_{k}\right), u \in U$, and define

$$
|x|:=:|m|=|a| \text {. }
$$

for $m=\left(\begin{array}{ll}a & 0 \\ 0 & a^{-1}\end{array}\right)$. Set

$$
F\left(\widetilde{x}, \phi_{1}, s\right)=\omega_{1}(\widetilde{x}) \phi_{1}(0)|x|^{s-1 / 2}
$$

Finally define

$$
E\left(\widetilde{x}, \phi_{1}, s\right)=\sum_{\gamma \in P(k) \backslash \mathrm{SL}_{2}(k)} F\left(\gamma \widetilde{x}, \phi_{1}, s\right) .
$$

Suppose that $V_{1}$ is anisotropic. Then the Eisenstein series is holomorphic at $s=1 / 2$.

Now by the Siegel-Weil formula, we obtain the main formula

$$
\ell_{H}(\varphi)=\int_{\mathrm{SL}_{2}(k) \backslash \mathrm{SL}_{2}(\mathbb{A})} f(x) \theta_{0}(\widetilde{x}) E\left(\widetilde{x}, \phi_{1}, 1 / 2\right) d x
$$

The right hand side is exactly the kind of integral considered by Gelbart and Jacquet $([6])$, which generalizes the classical Shimura integral. Unfolding this integral we get an Euler product which represents nothing but the symmteric square L-function of $f$. In a little more detail one has the following. Change $1 / 2$ 
to $s$ and call the right hand side $J(s)$ so that $\ell_{H}(\varphi)=J(1 / 2)$. We have

$$
\begin{aligned}
J(s) & =\int_{P(k) \backslash \mathrm{SL}_{2}\left(\mathbb{A}_{k}\right)} f(x) \theta_{0}(\widetilde{x}) F\left(\widetilde{x}, \phi_{1}, s\right) d x \\
& =\int_{N(k) \backslash \mathrm{SL}_{2}\left(\mathbb{A}_{k}\right)} f(x) \omega_{0}(\widetilde{x}) \phi_{0}(e) F\left(\widetilde{x}, \phi_{1}, s\right) d x \\
& =\int_{N(k) \backslash \mathrm{SL}_{2}\left(\mathbb{A}_{k}\right)} f(x) \omega(x) \phi(e)|x|^{s-1 / 2} d x \\
& =\int_{N\left(\mathbb{A}_{k}\right) \backslash \mathrm{SL}_{2}\left(\mathbb{A}_{k}\right)} \int_{N(k) \backslash N\left(\mathbb{A}_{k}\right)} f(n x) \omega(n x) \phi(e)|x|^{s-1 / 2} d n d x \\
& =\int_{N\left(\mathbb{A}_{k}\right) \backslash \mathrm{SL}_{2}\left(\mathbb{A}_{k}\right)} W_{-q(e)}(x) \omega(x) \phi(e)|x|^{s-1 / 2} d x .
\end{aligned}
$$

Here

$$
W_{-q(e)}(x)=\int_{k \backslash \mathbb{A}_{k}} f\left(\left(\begin{array}{ll}
1 & t \\
0 & 1
\end{array}\right) x\right) \psi(q(e) t) d t
$$

is the $-q(e)$-Fourier coefficient of $f$.

Lemma 4.3.1. Let e be a nonzero element in $V$ and let $H$ be the stabilizer of e in $G$. Let $\varphi$ be a cusp form on $G$ given by (4.3.1). Then

$$
\int_{H(k) \backslash H\left(\mathbb{A}_{k}\right)} \varphi d h=J(1 / 2, \varphi)
$$

where

$$
J(s, \varphi)=\int_{N\left(\mathbb{A}_{k}\right) \backslash \mathrm{SL}_{2}\left(\mathbb{A}_{k}\right)}|x|^{s-1 / 2} W_{-q(e)}(x)(\omega(x) \phi)(e) d x .
$$

Here $W_{-q(e)}(x)$ is the $-q(e)$-th Fourier coefficient of $f$ as defined above.

Proof. We have proved the case where $\phi=\phi_{0} \otimes \phi_{1}$. But the general case follows by the density of the factorizable Schwartz-Bruhat functions and the continuity of the integrals of Gelbart-Jacquet type ([6]).

4.4. Theta Lifts for Eisenstein Series. We use the notations in Sections 4.2 and 4.3. Consider the see-saw diagram (4.3.2)

$$
\begin{gathered}
O(V) \\
O\left(V_{0}\right) \times O\left(V_{1}\right)
\end{gathered} \mathrm{SL}^{\mathrm{SL}_{2} \times \mathrm{SL}_{2}}
$$

We shall show that formula (4.3.1) holds when $\varphi$ is an Eisenstein series of $\mathrm{O}(V)$. 
Since the space $V$ with norm is isomorphic to the direct sum of $F$ with norm $-\mathrm{N}_{F / k}$ and a hyperbolic space, $O(V)$ is the orthogonal group with respect to

$$
\left(\begin{array}{c}
1 \\
* * \\
* * \\
1
\end{array}\right)
$$

where $\left(\begin{array}{c}* * \\ * *\end{array}\right)$ defines the norm $-\mathrm{N}_{F / k}$ on $F$, we have only one non-trivial parabolic subgroup $P_{0}$ of $\mathrm{O}(V)$ defined over the number field $k$. We write $P_{0}=M_{0} N_{0}$, where

$$
\begin{aligned}
& M_{0}=\left\{m(a, b) \in \mathrm{GL}_{1} \times \mathrm{O}(F) \in \mathrm{O}(V)\right\} \\
& N_{0}=\left\{n(x)=\left(\begin{array}{rrr}
1 & x & * \\
I_{2} & x^{*} \\
& 1
\end{array}\right) \in \mathrm{O}(V)\right\} .
\end{aligned}
$$

The characters of $M_{0}(k) \backslash M_{0}\left(\mathbb{A}_{k}\right)$ are

$$
\chi(m(a, b))=\chi_{s}(a) \delta(b),
$$

where $\chi_{s}(a)=\chi_{0}(a)|a|^{s}$ and $\delta$ is a character of $\mathrm{O}\left(F, \mathbb{A}_{k}\right)$. The Eisenstein series attached to the normalized induced representation

$$
I(\chi)=\operatorname{Ind}_{P_{0}\left(\mathbb{A}_{k}\right)}^{\mathrm{O}(V)\left(\mathbb{A}_{k}\right)}(\chi)
$$

is defined by

$$
E\left(g, \alpha_{\chi}\right)=\sum_{\gamma \in P_{0}(k) \backslash O(V)(k)} \alpha_{\chi}(\gamma g)
$$

for $\alpha_{\chi} \in I(\chi)$.

Proposition 4.4.1. The Eisenstein series $E\left(g, \alpha_{\chi}\right)$ has a non-zero theta lift to $\mathrm{SL}_{2}$ if and only if the character $\delta$ is trivial. Moreover, the Eisenstein series $E\left(g, \alpha_{\chi_{s}}\right)$ is lifted to the Eisenstein series $\mathcal{E}\left(h, \beta_{s}\right)$, where

$$
\beta_{s} \in \operatorname{Ind}_{B\left(\mathbb{A}_{k}\right)}^{\mathrm{SL}_{2}\left(\mathbb{A}_{k}\right)}\left(\chi_{s}\right)
$$

Proof. This follows the calculation in Section 5.4, in Kudla-Rallis [14].

From this we can write, for $\chi=\chi_{s}$,

$$
E\left(g, \alpha_{\chi}\right)=\int_{\mathrm{SL}_{2}(k) \backslash \mathrm{SL}_{2}\left(\mathbb{A}_{k}\right)} \mathcal{E}\left(x, \beta_{s}\right) \theta(g, x ; \omega(z) \phi) d x
$$

where $\theta(g, h ; \omega(z) \phi)$ is the regularized theta function, which is rapidly decreasing in the variable $x$. Note that the integral in (4.4.4) converges absolutely as long as the theta function $\theta(g, x ; \phi)$ is rapidly decreasing in the variable $x$ for some choice 
of $\phi$. For generic value $s$, the induced representation $\operatorname{Ind}_{B\left(\mathbb{A}_{k}\right)}^{\mathrm{SL}_{2}\left(\mathbb{A}_{k}\right)}\left(\chi_{s}\right)$ is irreducible. Hence if the integral in (4.4.4) does not vanish, it must be the Eisenstein series as given in (4.4.4).

In order to calculate the period over $\mathrm{O}\left(V_{1}\right)(k) \backslash \mathrm{O}\left(V_{1}\right)\left(\mathbb{A}_{k}\right)$ of the Eisenstein series $E\left(g, \alpha_{\chi}\right)$, we consider the following choice of the test function $\phi$. We first consider the dual reductive pair $\left(\mathrm{O}\left(V_{0}\right), \widetilde{\mathrm{SL}}_{2}\right)$. By the regularization method in [14] and [10], there is an operator $z_{0}$ (in the center of the universal enveloping algebra or of the Hecke algebra for the dual reductive pair) such that for any test function $\phi_{0} \in \mathcal{S}\left(V_{0}(\mathbb{A})\right)$, the theta function $\theta\left(h, \widetilde{x} ; \omega\left(z_{0}\right) \phi_{0}\right)$ is rapidly decreasing in the variable $\widetilde{x}$ or more precisely on $\widetilde{\mathrm{SL}}_{2}(k) \backslash \widetilde{\mathrm{SL}}_{2}(\mathbb{A})$. Then we consider the dual reductive pair $\left(\mathrm{O}\left(V_{1}\right), \widetilde{\mathrm{SL}}_{2}\right)$. There is an operator $z_{1}$ such that for any test function $\phi_{1} \in \mathcal{S}\left(V_{1}(\mathbb{A})\right.$, the theta function $\theta\left(h, \widetilde{x} ; \omega\left(z_{1}\right) \phi_{1}\right)$ is rapidly decreasing in the variable $h$ or more precisely on $\mathrm{O}\left(V_{1}\right)(k) \backslash \mathrm{O}\left(V_{1}\right)(\mathbb{A})$. Note that if $V_{1}$ is $k$-anisotropic, then there is no need of regularization.

Now take $\phi=\omega\left(z_{0}\right) \phi_{0} \otimes \omega\left(z_{1}\right) \phi_{1} \in \mathcal{S}(V(\mathbb{A}))$. Then we have

$$
\theta\left(\left(h_{0}, h_{1}\right), x ; \phi\right)=\theta\left(h_{0}, \widetilde{x} ; \omega\left(z_{0}\right) \phi_{0}\right) \cdot \theta\left(h_{1}, \widetilde{x} ; \omega\left(z_{1}\right) \phi_{1}\right) .
$$

Note that the product of two genuine functions on $\widetilde{\mathrm{SL}}_{2}(\mathbb{A})$ factors through the linear group $\mathrm{SL}_{2}(\mathbb{A})$. Since $\theta\left(h_{0}, \widetilde{x} ; \omega\left(z_{0}\right)\right.$ is rapidly decreasing in $\widetilde{x}$ and $\theta\left(h_{1}, \widetilde{x} ; \omega\left(z_{1}\right) \phi_{1}\right)$ has moderate growth in $\widetilde{x}$, we know that the integral in (4.4.4) is equal to

$$
\mathcal{F}\left(\left(h_{0}, h_{1}\right), s\right)=\int_{\mathrm{SL}_{2}(k) \backslash \mathrm{SL}_{2}\left(\mathbb{A}_{k}\right)} \mathcal{E}\left(x, \beta_{s}\right) \theta\left(h_{0}, \widetilde{x} ; \omega\left(z_{0}\right) \phi_{0}\right) \theta\left(h_{1}, \widetilde{x} ; \omega\left(z_{1}\right) \phi_{1}\right) d x
$$

which converges absolutely. As remarked before, if integral (4.4.5) is non-zero, it gives the Eisenstein series $E\left(g, \alpha_{\chi}\right)$ for generic value $s$.

Next we compute the period of $\mathcal{F}\left(\left(h_{0}, h_{1}\right), s\right)$ over the variable $h_{1}$. By the see-saw duality, we have

$$
\begin{aligned}
& \int_{\left[\mathrm{O}\left(V_{1}\right)\right]} \mathcal{F}\left(\left(1, h_{1}\right)\right) d h_{1} \\
= & \int_{\left[\mathrm{O}\left(V_{1}\right)\right]} \int_{\left[\mathrm{SL}_{2}\right]} \mathcal{E}\left(x, \beta_{s}\right) \theta\left(1, x ; \omega\left(z_{0}\right) \phi_{0}\right) \theta\left(h_{1}, \widetilde{x} ; \omega\left(z_{1}\right) \phi_{1}\right) d x d h_{1} \\
= & \int_{\left[\mathrm{SL}_{2}\right]} \mathcal{E}\left(x, \beta_{s}\right) \theta\left(1, x ; \omega\left(z_{0}\right) \phi_{0}\right) \int_{\left[\mathrm{O}\left(V_{1}\right)\right]} \theta\left(h_{1}, \widetilde{x} ; \omega\left(z_{1}\right) \phi_{1}\right) d h_{1} d x
\end{aligned}
$$

where $[G]$ denotes the quotient $G(k) \backslash G(\mathbb{A})$. By the regularized Siegel-Weil formula (4.3.5) we have

$$
\int_{\left[\mathrm{O}\left(V_{1}\right)\right]} \theta\left(h_{1}, \widetilde{x} ; \omega\left(z_{1}\right) \phi_{1}\right) d h_{1}=\mathcal{E}\left(\widetilde{x}, \omega\left(z_{1}\right) \phi_{1}, \frac{1}{2}\right) .
$$


Hence we obtain

$$
\int_{\left[\mathrm{O}\left(V_{1}\right)\right]} \mathcal{F}\left(\left(1, h_{1}\right), s\right) d h_{1}=\int_{\left[\mathrm{SL}_{2}\right]} \mathcal{E}\left(x, \beta_{s}\right) \theta\left(1, \widetilde{x} ; \omega\left(z_{0}\right) \phi_{0}\right) \mathcal{E}\left(\widetilde{x}, \omega\left(z_{1}\right) \phi_{1}, \frac{1}{2}\right) d x .
$$

Note that since $\theta\left(1, \widetilde{x} ; \omega\left(z_{0}\right) \phi_{0}\right)$ is rapidly decreasing in $\widetilde{x}$, the integral in (4.4.7) converges absolutely. This is the analogue of (4.3.7) with the cusp form $f$ replaced by the Eisenstein series $\mathcal{E}\left(x, \beta_{s}\right)$. We want to show this integral is also eulerian. We can consider the following more general integral

$$
\int_{\mathrm{SL}_{2}(k) \backslash \mathrm{SL}_{2}\left(\mathbb{A}_{k}\right)} \mathcal{E}\left(x, \beta_{s}\right) \theta\left(1, \widetilde{x} ; \omega\left(z_{0}\right) \phi_{0}\right) \mathcal{E}\left(\widetilde{x}, \widetilde{\beta}_{\lambda}\right) d x .
$$

By unfolding the Eisenstein series $\mathcal{E}\left(\widetilde{x}, \widetilde{\phi}_{\lambda}\right)$, we have

$$
\int_{B(k) \backslash \mathrm{SL}_{2}\left(\mathbb{A}_{k}\right)} \mathcal{E}\left(x, \beta_{s}\right) \theta\left(1, \widetilde{x} ; \omega(z) \phi_{1}\right) \cdot \widetilde{\beta}_{\lambda}(\widetilde{x}) d x .
$$

In general we have

$$
\theta\left(1, \widetilde{x} ; \phi_{0}\right)=\omega(\widetilde{x}) \phi_{0}(0)+\sum_{\xi \in k^{\times}} \omega(\widetilde{x}) \phi_{0}(\widetilde{x}) .
$$

Because of the regularization, we have

$$
\omega(\widetilde{x}) \omega\left(z_{0}\right) \phi_{0}(0)=0
$$

identically. Hence we have

$$
\theta\left(1, \widetilde{x} ; \omega\left(z_{0}\right) \phi_{0}\right)=\sum_{\xi \in k^{\times}} \omega(\widetilde{x}) \omega\left(z_{0}\right) \phi_{0}(\xi) .
$$

Now by the same calculation as we did for Lemma 4.3.1, we obtain that for $\chi=\chi_{s}$, we have

$$
\int_{\mathrm{O}\left(V_{1}\right)(k) \backslash \mathrm{O}\left(V_{1}\right)\left(\mathbb{A}_{k}\right)} \mathcal{F}\left(\left(1, h_{1}\right), s\right) d h_{1}=J\left(\frac{1}{2}, E\left((1, \cdot), \beta_{\chi_{s}}\right)\right)
$$

where

$$
J\left(\lambda, E\left((1, \cdot), \alpha_{\chi_{s}}\right)\right)=\int_{N\left(\mathbb{A}_{k}\right) \backslash \mathrm{SL}_{2}\left(\mathbb{A}_{k}\right)}|x|^{\lambda-\frac{1}{2}} W_{-q(e), s}(x) \omega(x) \omega(z) \phi(e) d x .
$$

Here we define $W_{-q(e), s}(x)$ by

$$
W_{\beta, s}(x)=\int_{k \backslash \mathbb{A}_{k}} \mathcal{E}\left(\left(\begin{array}{ll}
1 & t \\
0 & 1
\end{array}\right) x, \phi_{s}\right) \psi(q(e) t) d t .
$$

As a consequence, we know that

$$
\mathcal{F}(g, s)=\int_{\mathrm{SL}_{2}(k) \backslash \mathrm{SL}_{2}\left(\mathbb{A}_{k}\right)} \mathcal{E}\left(x, \beta_{s}\right) \theta\left(g, x ; \omega\left(z_{0}\right) \phi_{0} \otimes \omega\left(z_{1}\right) \phi_{1}\right) d x
$$


is not identically zero as indicated in Lemma 4.3.1. Therefore we obtain the analogue of Lemma 4.3.1 for the period of Eisenstein series

Lemma 4.4.2. For $\chi=\chi_{s}$, we have

$$
\begin{aligned}
\int_{\mathrm{O}\left(V_{1}\right)(k) \backslash \mathrm{O}\left(V_{1}\right)\left(\mathbb{A}_{k}\right)} E\left(\left(1, h_{1}\right), \alpha_{\chi_{s}}\right) d h_{1} & =\int_{\mathrm{O}\left(V_{1}\right)(k) \backslash \mathrm{O}\left(V_{1}\right)\left(\mathbb{A}_{k}\right)} \mathcal{F}\left(\left(1, h_{1}\right), s\right) d h_{1} \\
& =J\left(\frac{1}{2}, E\left((1, \cdot), \beta_{\chi_{s}}\right)\right) .
\end{aligned}
$$

\section{Periods of CUSP FORMS}

In this section we shall prove Theorem 1.2.1 for cusp forms using theta lifting method. Following section 4.3, it remains to compute the Gelbart-Jacquet integrals. However, we will start with a direct evaluation in the case where $H$ is the split subgroup $\mathrm{PGL}_{2}(k)$ of $\mathrm{PGL}_{2}(F)$.

5.1. Split case. Let us first compute the period for the case where $H$ is the subgroup $\mathrm{PGL}_{2}(k)$ of $\mathrm{PGL}_{2}(F)$. Let $\varphi$ be the newform in a spherical cuspidal representation $\pi$ on $\mathrm{PGL}_{2}\left(\mathbb{A}_{F}\right)$ so that its Mellin transformation defines the right L-series as in (1.2.1). We want to study the following period:

$$
\ell_{H}(\varphi)=\int_{H(k) \backslash H\left(\mathbb{A}_{k}\right)} \varphi(h) d h .
$$

We have shown in section 4.3 that the non-vanishing of the periods implies that $\pi$ is a base change of a representation $\sigma$ of $\mathrm{GL}_{2}\left(\mathbb{A}_{k}\right)$. In other words if locally at each place $v$ of $k, \sigma_{v}$ is given by a principal series $\pi\left(\chi_{v}, \chi_{v}^{-1} \eta_{v}\right)$ with $\chi_{v}$ unramified, then at a place $w$ of $F$ over $v, \pi_{w}$ is given by

$$
\pi\left(\chi_{v} \circ \mathrm{N}_{w / v}, \chi_{v}^{-1} \circ \mathrm{N}_{w / v}\right) .
$$

Let $E(g, s)$ be a spherical Eisenstein series on $H\left(\mathbb{A}_{k}\right)$ attached to the induced representation $\pi\left(|\cdot|^{s},|\cdot|^{-s}\right)$ :

$$
E(g, s)=\sum_{\gamma \in B(k) \backslash H(k)} f(\gamma g, s)
$$

where for $g=\left(\begin{array}{ll}a & x \\ 0 & b\end{array}\right) u$ with $u \in U_{H}$,

$$
f(g, s)=|a / b|^{s+1 / 2} .
$$

Now we have

$$
\ell_{H}(\varphi)=c_{1} \cdot \operatorname{Res}_{s=1 / 2} \int_{H(k) \backslash H\left(\mathbb{A}_{k}\right)} \varphi(h) E(g, s) d h,
$$


where $c_{1}$ is the constant so that $c_{1} E(g, s)$ has residue equal to 1 at $s=1 / 2$. Unfold the above integral to obtain:

$$
\begin{aligned}
\ell(\varphi) & =c_{1} \cdot \operatorname{Res}_{s=1 / 2} \int_{B(k) \backslash H\left(\mathbb{A}_{k}\right)} \varphi(h) f(g, s) d h \\
& =c_{1} \cdot \operatorname{Res}_{s=1 / 2} \int_{N\left(\mathbb{A}_{k}\right) B(k) \backslash H\left(\mathbb{A}_{k}\right)} \int_{N(k) \backslash N\left(\mathbb{A}_{k}\right)} \varphi(n h) d n f(h, s) d h,
\end{aligned}
$$

where $d h$ is the quotient measure on $N\left(\mathbb{A}_{k}\right) B(k) \backslash H\left(\mathbb{A}_{k}\right)$.

Let $\psi$ be any nontrivial character of $N(k) \backslash N\left(\mathbb{A}_{k}\right)$. Let $W$ be the Whittacker coefficient of $\varphi$ for the character $x \mapsto \psi\left(\operatorname{tr}_{F / k} x\right)$ :

$$
W(g)=\int_{N(F) \backslash N\left(\mathbb{A}_{F}\right)} \varphi\left(\left(\begin{array}{ll}
1 & x \\
0 & 1
\end{array}\right) g\right) \psi\left(-\operatorname{tr}_{F / k} x\right) d x .
$$

Then $\varphi$ has a Fourier expansion

$$
\varphi\left(\left(\begin{array}{ll}
1 & x \\
0 & 1
\end{array}\right) g\right)=\sum_{\xi \in F^{\times}} \psi(\operatorname{tr}(x \xi)) \cdot W\left(\left(\begin{array}{l}
\xi \\
1
\end{array}\right) g\right) .
$$

Bring this to the inner integral to obtain

$$
\int_{N(k) \backslash N\left(\mathbb{A}_{k}\right)} \varphi(n h) d n=\sum_{\xi \in k^{\times}} W\left(\left(\begin{array}{c}
\xi_{0} \xi \\
\\
\end{array}\right) h\right)
$$

where $\xi_{0}$ is any trace free element in $F^{\times}$. Now via the surjective maps

$$
M\left(\mathbb{A}_{k}\right) U_{H} \longrightarrow N\left(\mathbb{A}_{k}\right) \backslash H\left(\mathbb{A}_{k}\right),
$$

the quotient measure $d h$ on $N\left(\mathbb{A}_{k}\right) \backslash H\left(\mathbb{A}_{k}\right)$ is lifted to the product measure $c_{2} \delta(m)^{-1} d m \cdot d u$ where $c_{2}$ is a positive constant and $d u$ is a measure on $U_{H}$ with volume 1 . Thus we obtain the following expression:

$$
\ell(\varphi)=c_{1} \cdot c_{2} \cdot \operatorname{Res}_{s=1 / 2} \int_{\mathbb{A}_{k}^{\times}} W\left(\left(\begin{array}{cc}
\xi_{0} a & 0 \\
0 & 1
\end{array}\right)\right)|a|^{s-1 / 2} d a .
$$

The last integral is a product of local integrals:

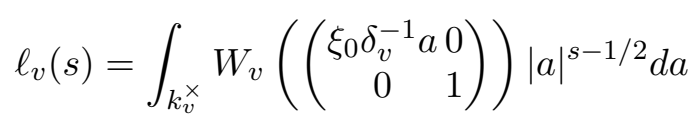

where $W_{v}$ is the Whittaker function for $\pi_{v}$ with respect to an unramified additive character of $F_{v}$. We want to evaluate this integral case by case. 
First assume that $v$ is a finite place which is unramified and nonsplit in $F$. Let $w$ be the place of $F$ over $v$. Then

$$
\begin{aligned}
& \ell_{v}(s)=\sum W_{v}\left(\left(\begin{array}{cc}
\xi_{0} \delta_{v}^{-1} \pi_{v}^{n} & 0 \\
0 & 1
\end{array}\right)\right)\left|\pi_{v}^{n}\right|_{v}^{s-1 / 2} \\
& =\sum W_{v}\left(\left(\begin{array}{cc}
\xi_{0} \delta_{v}^{-1} \pi_{v}^{n} & 0 \\
0 & 1
\end{array}\right)\right)\left|\pi_{v}^{n}\right|_{w}^{s / 2-1 / 4}
\end{aligned}
$$

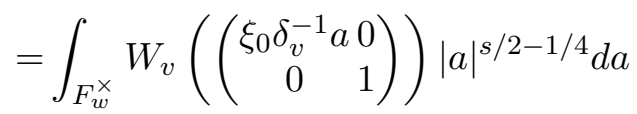

$$
\begin{aligned}
& =\left|\xi_{0} \delta_{v}^{-1}\right|^{1 / 4-s / 2} \int_{F_{w}^{\times}} W_{v}\left(\left(\begin{array}{ll}
a & 0 \\
0 & 1
\end{array}\right)\right)|a|^{s / 2-1 / 4} d a \\
& =\left|\xi_{0} \delta_{v}^{-1}\right|^{1 / 4-s / 2} L\left(s / 2+1 / 4, \pi_{v}\right) \text {. }
\end{aligned}
$$

If we write $\sigma_{v}=\pi\left(\chi_{v}, \chi_{v}^{-1} \cdot \eta_{v}\right)$ then $\pi_{w}=\pi\left(\chi_{v} \circ \mathrm{N}_{w / v}, \chi_{v}^{-1} \circ \mathrm{N}_{w / v}\right)$. Thus

$$
\begin{aligned}
L\left(s, \pi_{v}\right) & =\left(1-\chi_{v}\left(\pi_{v}^{2}\right)\left|\pi_{v}\right|_{v}^{2 s}\right)^{-1}\left(1-\chi_{v}^{-1}\left(\pi_{v}^{2}\right)\left|\pi_{v}\right|_{v}^{2 s}\right)^{-1} \\
& =L\left(2 s, \operatorname{sym}^{2} \sigma_{v}\right)\left(1+\left|\pi_{v}\right|_{v}^{2 s}\right) \\
& =L\left(2 s, \operatorname{sym}^{2} \sigma_{v}\right) \zeta_{v}(2 s) \zeta_{v}(4 s)^{-1} .
\end{aligned}
$$

It follows that

$$
\ell_{v}(s)=\left|\xi_{0} \delta_{v}^{-1}\right|^{1 / 4-s / 2} L\left(s+1 / 2, \operatorname{sym}^{2} \sigma_{v}\right) \zeta_{v}(s+1 / 2) \zeta_{v}(2 s+1)^{-1} .
$$

The case where $v$ is ramified in $F$ is similar:

$$
\begin{aligned}
\ell_{v}(s) & =\sum W_{v}\left(\left(\begin{array}{cc}
\xi_{0} \delta_{v}^{-1} \pi_{w}^{2 n} & 0 \\
0 & 1
\end{array}\right)\right)\left|\pi_{w}^{n}\right|_{w}^{s-1 / 2} \\
& =\left|\xi_{0} \delta_{v}^{-1}\right|^{1 / 4-s / 2} \sum W_{v}\left(\left(\begin{array}{cc}
\pi_{w}^{2 n} & 0 \\
0 & 1
\end{array}\right)\right)\left|\pi_{w}^{2 n}\right|_{w}^{s-1 / 2} \\
& =\left|\xi_{0} \delta_{v}^{-1}\right|^{1 / 4-s / 2} \int_{F_{w}^{\times}} W_{v}\left(\left(\begin{array}{cc}
a & 0 \\
0 & 1
\end{array}\right)\right)|a|_{w}^{s / 2-1 / 4} \frac{1+\nu(a)}{2} d a \\
& =\frac{1}{2}\left|\xi_{0} \delta_{v}^{-1}\right|^{1 / 4-s / 2}\left(L\left(s / 2+1 / 4, \pi_{v}\right)+L\left(s / 2+1 / 4, \nu, \pi_{v}\right)\right)
\end{aligned}
$$

where $\nu$ is the character on $F_{w}^{\times}$given by

$$
\nu(x)=(-1)^{\text {ord } x} .
$$

If we write $\sigma_{v}=\pi\left(\chi_{v}, \chi_{v}^{-1} \cdot \eta_{v}\right)$ with $\chi_{v}$ unramified, then

$$
\pi_{w}=\pi\left(\chi_{v} \circ \mathrm{N}_{w / v}, \chi_{v}^{-1} \circ \mathrm{N}_{w / v}\right) .
$$


Thus

$$
\begin{aligned}
& L\left(s, \pi_{v}\right)+L\left(s, \nu, \pi_{v}\right) \\
= & \frac{1}{\left(1-\chi_{v}\left(\pi_{v}\right)\left|\pi_{v}\right|_{v}^{s}\right)\left(1-\chi_{v}^{-1}\left(\pi_{v}\right)\left|\pi_{v}\right|_{v}^{s}\right)}+\frac{1}{\left(1+\chi_{v}\left(\pi_{v}\right)\left|\pi_{v}\right|_{v}^{s}\right)\left(1+\chi_{v}^{-1}\left(\pi_{v}\right)\left|\pi_{v}\right|_{v}^{s}\right)} \\
= & \frac{2\left(1+\left|\pi_{v}\right|^{2 s}\right)}{\left(1-\chi_{v}\left(\pi_{v}\right)^{2}\left|\pi_{v}\right|^{2 s}\right)\left(1-\chi_{v}\left(\pi_{v}\right)^{-2}\left|\pi_{v}\right|^{2 s}\right)} \\
= & 2 \zeta_{v}(2 s) \zeta_{v}(4 s)^{-1} \cdot\left|\pi_{v}\right|^{s} L\left(2 s, \operatorname{Sym}^{2} \sigma_{v}\right) .
\end{aligned}
$$

It follows that

$$
\ell_{v}(s)=\left|\xi_{0} \delta_{v}^{-1}\right|^{1 / 4-s / 2} \zeta_{v}(s+1 / 2) \zeta(2 s+1)^{-1} L\left(s+1 / 2, \operatorname{Sym}^{2} \sigma_{v}\right) .
$$

Now we consider the case where $v$ is split in $F$. Let $w_{1}$ and $w_{2}$ be two places of $F$ over $v$. Then

$$
\begin{aligned}
\ell_{v}(s) & =\int_{k_{v}^{\times}} W_{w_{1}}\left(\left(\begin{array}{rr}
\xi_{01} \delta_{v}^{-1} & a \\
0 & 1
\end{array}\right)\right) W_{w_{2}}\left(\left(\begin{array}{rr}
-\xi_{01} \delta_{v}^{-1} a & 0 \\
0 & 1
\end{array}\right)\right)|a|^{s-1 / 2} d a \\
& =\left|\xi_{0} \delta_{v}^{-1}\right|^{1 / 4-s / 2} \int_{k_{v}^{\times}} W_{w_{1}}\left(\left(\begin{array}{ll}
a & 0 \\
0 & 1
\end{array}\right)\right) W_{w_{2}}\left(\left(\begin{array}{rr}
-a & 0 \\
0 & 1
\end{array}\right)\right)|a|^{s-1 / 2} d a \\
& =\left|\xi_{0} \delta_{v}^{-1}\right|_{v}^{1 / 4-s / 2} \zeta_{v}(2 s+1)^{-1} L\left(s+1 / 2, \pi_{w_{1}} \times \pi_{w_{2}}\right) .
\end{aligned}
$$

Here $L\left(s, \pi_{w_{1}} \times \pi_{w_{2}}\right)$ is the convolution of two L-functions $L\left(s, \pi_{w_{1}}\right)$ and $L\left(s, \pi_{w_{2}}\right)$. If $\sigma_{v}=\pi\left(\chi_{v}, \chi_{v}^{-1}\right)$ then

$$
L\left(s, \pi_{w_{1}}\right)=L\left(s, \pi_{w_{2}}\right)=L\left(s, \sigma_{v}\right)=\frac{1}{\left(1-\chi_{v}\left(\pi_{v}\right)\left|\pi_{v}\right|^{s}\right)\left(1-\chi_{v}\left(\pi_{v}\right)^{-1}\left|\pi_{v}\right|^{s}\right)}
$$

and

$$
\begin{aligned}
L\left(s, \pi_{w_{1}} \times \pi_{w_{2}}\right) & =\frac{1}{\left(1-\chi_{v}^{2}\left(\pi_{v}\right)\left|\pi_{v}\right|^{s}\right)\left(1-\chi_{v}^{-2}\left(\pi_{v}\right)\left|\pi_{v}\right|^{s}\right)\left(1-\left|\pi_{v}\right|^{s}\right)^{2}} \\
& =\zeta_{v}(s) L\left(s, \operatorname{Sym}^{2} \sigma_{v}\right) .
\end{aligned}
$$

It follows that

$$
\ell_{v}(s)=\left|\xi_{0} \delta_{v}^{-1}\right|_{v}^{1 / 4-s / 2} L\left(s+1 / 2, \operatorname{Sym}^{2} \sigma_{v}\right) \zeta_{v}(s+1 / 2) \zeta_{v}(2 s+1)^{-1} .
$$

In summary, we have shown that

$$
\begin{aligned}
\ell_{H}(\varphi) & =c_{1} \cdot c_{2} \cdot \operatorname{Res}_{s=1 / 2}\left|\xi_{0} \delta^{-1}\right|{ }^{1 / 4-s / 2} L\left(s+1 / 2, \operatorname{Sym}^{2} \sigma\right) \frac{\zeta_{k}(s+1 / 2)}{\zeta_{k}(2 s+1)} \\
& =c_{1} \cdot c_{2} \cdot c_{3} \cdot L\left(1, \operatorname{Sym}^{2} \sigma\right)
\end{aligned}
$$

where $c_{3}$ is the constant $\frac{\operatorname{Res}_{s=1} \zeta_{k}(s)}{\zeta_{k}(2)}$.

All constants $c_{1}, c_{2}$ and $c_{3}$ can be computed as in $\S 3.2$ and $\S 3.3$. Then we obtain Theorem 1.2.1 for split $H$. Alternatively, we may use the above method 
to compute continuous spectrum $\varphi=\int_{\Omega} c_{\mu} E_{\mu}$ where $E_{\mu}$ are newforms for representation $\pi\left(\mu, \mu^{-1}\right)$ with unitary character $\mu$ of $\mathbb{A}_{F}^{\times}$. Then we get the same expression except an additional form

$$
\operatorname{Res}_{s=1 / 2} \int_{\mathbb{A}_{k}^{\times}} c_{\phi}\left(\begin{array}{ll}
a & 0 \\
0 & 1
\end{array}\right)|a|^{s-1 / 2} d^{\times} a
$$

where $c_{\phi}$ is the constant term of $\phi$. One may show that the above Mellin transform of $c_{\phi}$ is holomorphic in $s$. Thus it has no residue at $s=1 / 2$. Since we already prove the main formula in Theorem 1.2.1, the constant $c_{1} \cdot c_{2} \cdot c_{3}$ must agree with the constant given in Theorem 1.2.1.

5.2. Gelbart-Jacquet integrals. Let us first recall what have been done so far for periods of cusp forms toward the proof of Theorem 1.2.1. We start with the setting in $\S 1$. Recall that we fix a quadratic extension of totally real fields $F / k$ and a quaternion algebra $D$ over $k$ embedded into $M_{2}(F)$.

Now let $\tau \in \mathrm{GL}_{2}\left(\mathbb{A}_{F}\right)$ with the Mumford-Tate group $D^{\times} / k^{\times}$, such that the right multiplication of $\tau$ defines a map of complex manifolds:

$$
\iota_{\tau}: \quad M_{U_{H}}:=H(k) \backslash Y \times H(\widehat{k}) / U_{H} \longrightarrow M_{U}:=\mathrm{PGL}_{2}(F) \backslash X \times \mathrm{PGL}_{2}(\widehat{F}) / U
$$

where $U$ is the standard maximal compact subgroup of $\mathrm{PGL}_{2}(\widehat{F})$ and $U_{H}$ is some maximal compact subgroup of $H(\widehat{k})$, respectively. Let $\varphi$ be the newform in a spherical cuspidal representation $\pi$ on $\mathrm{PGL}_{2}\left(\mathbb{A}_{F}\right)$ so that its Mellin transformation defines the right L-series as in (1.2.1). We want to study the following period:

$$
\ell_{H, \tau}(\varphi)=\int_{H(k) \backslash H\left(\mathbb{A}_{k}\right)} \varphi(h \tau) d h .
$$

In the last section we have shown that the non-vanishing of this integral implies that $\varphi$ is the theta lifting of a form $f$ on $\mathrm{GL}_{2}\left(\mathbb{A}_{k}\right)_{+}$. More precisely, let $G=\mathrm{GSO}(V)$ be the connected component of the group $\mathrm{GO}(V)$ of orthogonal similitudes of the space $V$ of $2 \times 2$ hermitian matrices over $F$ with norm given by determinant. We have shown in $\S 4.1$ that $G$ is generated by scalars and $\mathrm{GL}_{2}(F)$. Here the action of $\mathrm{GL}_{2}(F)$ on Hermitian matrices is given as usual:

$$
\gamma(A)=\gamma \cdot A \cdot \bar{\gamma}^{t}, \quad \forall \gamma \in \mathrm{GL}_{2}(F), \forall A \in V .
$$

Thus $G$ has the center generated by scalars and has the adjoint group $\operatorname{Res}_{F / k} \mathrm{PGL}_{2}$. We can view $\varphi$ as a form on $G\left(\mathbb{A}_{k}\right)$. The embedding

$$
H=D^{\times} / k^{\times} \longrightarrow \operatorname{Res}_{F / k} \operatorname{PGL}(2)
$$

can be lifted to an embedding to $G$ with action of $H$ on $V$ as follows:

$$
h(A)=\operatorname{det} h^{-1} \cdot h \cdot A \cdot \bar{h}^{t}, \quad \forall h \in D^{\times} .
$$


In this way, $H$ becomes the stabilizer of a vector $e \in V$ in the special group $\mathrm{SO}(V)$ with nontrivial norm. Moreover, let $V_{1}$ be the orthogonal complement of $V_{0}:=k e$. Then $H=\mathrm{SO}\left(V_{1}\right)$. Now view $\tau$ as an element in $G(\mathbb{A})$ and $g \mapsto \varphi(g \tau)$ as a function $\varphi_{\tau}$ on $G\left(\mathbb{A}_{k}\right)$.

Assume that the period $\ell_{H, \tau}(\varphi) \neq 0$. The main result of $\S 4.2$ shows that $\pi$ is the theta lifting of a representation $\sigma$ on $\mathrm{GL}_{2}\left(\mathbb{A}_{k}\right)_{+}$. Thus we have $\phi \in \mathcal{S}(V(\mathbb{A}))$ and $f \in V_{\sigma}$ such that (4.3.1) holds:

$$
\varphi(g)=\int_{\mathrm{SL}(2, k) \backslash \mathrm{SL}\left(2, \mathbb{A}_{k}\right)} \theta_{\phi}\left(g, x x_{0}\right) f\left(x x_{0}\right) d x,
$$

for any $g \in G\left(\mathbb{A}_{k}\right)$ where $x_{0} \in \mathrm{GL}\left(2, \mathbb{A}_{k}\right)$ is any element with the norm equal to the similitude of $g$. It is easy to see from (4.2.8) that the function $g \mapsto \varphi(g \tau)$ is given by the pair $\left(\omega\left(\tau, h_{0}\right) \phi, r\left(h_{0}\right) f\right)$ where $h_{0} \in \mathrm{GL}\left(2, \mathbb{A}_{k}\right)_{+}$with the same norm as $\tau$. Now applying Lemma (4.3.1) we obtain

$$
\ell_{H, \tau}(\varphi)=J(1 / 2)
$$

where $J(s)$ is a Gelbart-Jacquet integral:

$$
J(s)=\int_{N\left(\mathbb{A}_{k}\right) \backslash \mathrm{SL}_{2}\left(\mathbb{A}_{k}\right)}|x|^{s-1 / 2} W_{-q(e)}\left(x h_{0}\right)\left(\omega\left(\tau, x h_{0}\right) \phi\right)(e) d x .
$$

Recall that the measure $d x$ on $N\left(\mathbb{A}_{k}\right) \backslash \mathrm{SL}_{2}\left(\mathbb{A}_{k}\right)$ is taken to be the quotient measure of the measure $d h$ on $\mathrm{SL}_{2}\left(\mathbb{A}_{k}\right)$ and a measure $d n$ on $N\left(\mathbb{A}_{k}\right)$ so that the volumes of $\mathrm{SL}_{2}(k) \backslash \mathrm{SL}_{2}\left(\mathbb{A}_{k}\right)$ and $N(k) \backslash N\left(\mathbb{A}_{k}\right)$ are both equal to 1 .

Now we further assume that $f$ is the restriction of a cuspidal eigenform on $\mathrm{GL}_{2}\left(\mathbb{A}_{k}\right)$ with central character $\eta$, has weight 0 at infinite places, and is invariant under matrices $U_{v}\left(\varpi_{v}^{c_{v}}\right)$ of the form

$$
\left(\begin{array}{ll}
a & b \\
0 & 1
\end{array}\right) \in \mathrm{GL}_{2}\left(\mathcal{O}_{v}\right)
$$

The Whittaker function of $W$ with respect to a fixed character $\psi$ of $k \backslash \mathbb{A}_{k}$ has a decomposition $W=\otimes_{v} W_{v}$. These local Whittaker function $W_{v}$ are normalized such that

$$
W_{v}\left(\begin{array}{cc}
\delta_{v}^{-1} & 0 \\
0 & 1
\end{array}\right)=1
$$

Here $\delta_{v}$ are conductors of $\psi_{v}$.

The function $\phi$ can be chosen to be invariant under the standard maximal compact subgroups of $G\left(\mathbb{A}_{k}\right)$ and $\mathrm{SL}_{2}\left(\mathbb{A}_{k}\right)$ and to be decomposable: $\phi=\otimes \phi_{v}$. In this way, the function $J(s)$ has decomposition $J(s)=\prod J_{v}(s)$ with

$$
J_{v}(s)=\int_{N\left(k_{v}\right) \backslash S \mathrm{SL}_{2}\left(k_{v}\right)}|x|^{s-1 / 2} W_{-q(e), v}\left(x h_{0, v}\right)\left(\omega\left(\tau_{v}, x h_{0, v}\right) \phi_{v}\right)\left(e_{v}\right) d x .
$$


Here $W_{-q(e), v}$ is the normalized spherical Whittaker function for the representation $\sigma_{v}$ with respect to the character $x \longrightarrow \psi_{v}(\epsilon x)$. Of course the definition of function $J_{v}$ depends on the choice of $\psi_{v}, \tau_{v}, e_{v}, \phi_{v}$, and the decomposition of measures on $N\left(k_{v}\right) \backslash \mathrm{SL}_{2}\left(k_{v}\right)$.

In the following we assume that $\tau$ is normalized by formula (2.1.2) and (2.1.3). We want to show that $J_{v}(s)$ depends only on $\psi_{v}, \phi_{v}$, and the ramification of $H$ at $v$. Take $h_{0, v}=\left(\begin{array}{cc}1 & 0 \\ 0 & \lambda(\tau)\end{array}\right)$ with $\lambda(\tau)=\mathrm{N}_{F / k} \operatorname{det} \tau$. Assume that $|\lambda(\tau)|=1$ and make the substitution $x \longrightarrow h_{0} x h_{0}^{-1}$. Then we have

$$
J_{v}(s)=\int_{N\left(k_{v}\right) \backslash \mathrm{SL}_{2}\left(k_{v}\right)}|x|^{s-1 / 2} W_{\frac{-q(e)}{\lambda(\tau)}, v}(x)\left(\omega(x) \phi_{v}\right)\left(\tau_{v}^{-1} e_{v}\right) d x .
$$

Suppose $H^{\prime}$ is another group defined by $\epsilon^{\prime}$ with the same ramification as $H$ at $v$. Then $\epsilon^{\prime}=\mathrm{N}(t) \epsilon$ for some $t \in F_{v}$. It follows that

$$
H^{\prime}=\alpha H \alpha^{-1}, \quad e_{v}^{\prime}=\alpha e_{v}
$$

with $\alpha=\left(\begin{array}{c}t \\ 1\end{array}\right)$. Since $\operatorname{Ad}\left(\tau_{v}^{\prime}\right)\left(U_{v}\right) \cap H^{\prime}\left(k_{v}\right)$ is maximal compact in $H^{\prime}\left(k_{v}\right)$, $\operatorname{Ad}\left(\alpha^{-1} \tau_{v}^{\prime}\right)\left(U_{v}\right) \cap H\left(k_{v}\right)$ is maximal in $H\left(k_{v}\right)$. It follows that

$$
\alpha^{-1} \tau_{v}^{\prime} \in H\left(k_{v}\right) \cdot \tau_{v} U_{v} .
$$

As $\phi_{v}$ is invariant under $U_{v}$,

$$
\phi_{v}\left(\left(\tau^{\prime}\right)^{-1} e_{v}^{\prime}\right)=\phi_{v}\left(\tau_{v} e_{v}\right) .
$$

This is enough to conclude that $J_{v}(s)$ is same for $H^{\prime}$ as

$$
\frac{-q(e)}{\lambda(\tau)}=\frac{-\delta_{D}}{\delta_{F}} \quad\left(\bmod \mathcal{O}_{v}^{\times}\right)
$$

by Lemma 3.2.4.

Now define local periods by the formula

$$
j\left(H_{v}, \sigma_{v}, \phi_{v}, \psi_{v}\right)=J_{v}(1 / 2) .
$$

where $H_{v}$ is the localization of $H$ at $v$. Thus we have the decomposition:

$$
\ell_{H, \tau}\left(\theta_{f}^{\phi}\right)=c \prod_{v} j\left(H_{v}, \sigma_{v}, \phi_{v}, \psi_{v}\right) .
$$

Here $c$ is a constant related to the decomposition of measures on $\mathrm{SL}_{2}\left(\mathbb{A}_{k}\right)$.

By definition, the dependence on $\psi_{v}$ of $j\left(H_{v}, \sigma_{v}, \phi_{v}, \psi_{v}\right)$ is through the norm $\left|\delta_{v}\right|$ of the conductor of $\psi_{v}$. In the following we want to show that it depends essentially on the parity class in $k_{v}^{\times} / \mathcal{O}_{v}^{\times} \cdot\left(k_{v}^{\times}\right)^{2}$. Here $\mathcal{O}_{v}^{\times}= \pm 1$ for archimedean place $v$. Let $\psi_{v}^{\prime}(x)=\psi_{v}\left(t^{2} x\right)$ be a different character and $\phi_{v}^{\prime}$ be another function 
invariant under the maximal compact subgroups of $G_{v}$ and $\mathrm{SL}_{v}(2)$ with respect to the Weil representation $\omega^{\prime}$ defined by the character $\psi_{v}^{\prime}$. Since

$$
\omega^{\prime}(x)=\omega\left(m(t) x m(t)^{-1}\right)
$$

we have

$$
\begin{aligned}
& j\left(H_{v}, \sigma_{v}, \phi_{v}^{\prime}, \psi_{v}^{\prime}\right) \\
& =\int_{N\left(k_{v}\right) \backslash \mathrm{SL}_{2}\left(k_{v}\right)}|x|^{s-1 / 2} W_{\frac{-q(e)}{\lambda(\tau)}, v}^{\prime}(x)\left(\omega^{\prime}(x) \phi_{v}^{\prime}\right)\left(\tau_{v}^{-1} e_{v}\right) d x \\
& =\int_{N\left(k_{v}\right) \backslash \mathrm{SL}_{2}\left(k_{v}\right)}|x|^{s-1 / 2} W_{\frac{-q(e)}{\lambda(\tau)}, v}\left(\left(\begin{array}{cc}
t^{2} & 0 \\
0 & 1
\end{array}\right) x\right)\left(\omega\left(m(t) x m(t)^{-1}\right) \phi_{v}^{\prime}\right)\left(\tau_{v}^{-1} e_{v}\right) d x \\
& =\eta(t) \int_{N\left(k_{v}\right) \backslash \mathrm{SL}_{2}\left(k_{v}\right)}|x|^{s-1 / 2} W_{\frac{-q(e)}{\lambda(\tau)}, v}(m(t) x)\left(\omega\left(m(t) x m(t)^{1}\right) \phi_{v}^{\prime}\right)\left(\tau_{v}^{-1} e_{v}\right) d x \\
& =\eta(t)|t|^{5 / 2-s} \int_{N\left(k_{v}\right) \backslash \mathrm{SL}_{2}\left(k_{v}\right)}|x|^{s-1 / 2} W_{\frac{-q(e)}{\lambda(\tau)}, v}(x)\left(\omega\left(x m(t)^{-1}\right) \phi_{v}^{\prime}\right)\left(\tau_{v}^{-1} e_{v}\right) d x .
\end{aligned}
$$

Thus we have the following expression

$$
j\left(H_{v}, \sigma_{v}, \phi_{v}^{\prime}, \psi_{v}^{\prime}\right)=\eta(t)|t|^{2} j\left(H_{v}, \sigma_{v}, \omega\left(m(t)^{-1}\right) \phi_{v}^{\prime}, \psi_{v}\right) .
$$

Now we want to evaluate $J_{v}(s)$ in the unramified case defined by the following conditions:

- $\eta_{v}$ is unramified; here $\eta$ is the quadratic character corresponding to the extension of $F / k$.

- $\phi_{v}$ is the characteristic function of $\delta_{v}^{-1} V\left(\mathcal{O}_{v}\right)$;

- $H$ is split at $v$.

In the unramified case, we may assume $\epsilon=1$ and $\tau=1$. Consider the Iwasawa decomposition

$$
\mathrm{SL}_{2}\left(k_{v}\right)=N\left(k_{v}\right) M\left(k_{v}\right) U_{v}^{\prime}
$$

and the integration formula

$$
d x=|x|^{-2} d n d m d u, \quad n \in N\left(k_{v}\right), m \in M\left(k_{v}\right), u \in U_{v}^{\prime} .
$$

Here $d n$ is the invariant measure on $N\left(k_{v}\right)$ with respect to the additive character on $N\left(k_{v}\right)$ such that $N\left(\mathcal{O}_{v}\right)$ has volume 1 , and $d m$ is the invariant measure on $M\left(k_{v}\right)$ such that the volume of $M\left(\mathcal{O}_{v}\right)$ has volume 1 , and $d u$ is the measure on 
the maximal compact subgroup $U_{v}^{\prime}$ of $\mathrm{SL}_{2}\left(k_{v}\right)$ with volume 1 . Then we obtain

$$
\begin{aligned}
J_{v}(s) & =\int_{k_{v}^{\times}} \eta(a)|a|^{s-1 / 2} W_{v}\left(\left(\begin{array}{ll}
a & 0 \\
0 & a^{-1}
\end{array}\right) u\right) \phi(a e) d^{\times} a \\
& =\int_{k_{v}^{\times}}|a|^{s-1 / 2} W_{v}\left(\left(\begin{array}{cc}
a^{2} & 0 \\
0 & 1
\end{array}\right)\right) \phi_{v}(a e) d^{\times} a . \\
& =\int_{k_{v}^{\times}}|a|_{v}^{s-1 / 2} W_{v}\left(\begin{array}{cc}
a^{2} & 0 \\
0 & 1
\end{array}\right) d^{\times} a .
\end{aligned}
$$

Let $\alpha$ and $\beta$ be the parameters of the local L-factors of $f$ on $\mathrm{GL}_{2}$ below, with $\alpha \beta=\eta_{v}(\varpi)$. Let $\chi$ be the character on $k_{v}^{\times}$given by $\chi(x)=(-1)^{\operatorname{ord}(x)}$. Then we have

$$
L_{v}(s, f)=\frac{1}{\left(1-\alpha|\varpi|^{s}\right)\left(1-\beta|\varpi|^{s}\right)}, \quad L_{v}(s, f, \chi)=\frac{1}{\left(1+\alpha|\varpi|^{s}\right)\left(1+\beta|\varpi|^{s}\right)} .
$$

On the other hand, these local L-factors are expressed as the local Mellin transforms of the local Whittaker functions by

$$
\begin{gathered}
L_{v}(s, f)=\int_{k_{v}^{\times}} W_{v}\left(\left(\begin{array}{cc}
a \delta_{v}^{-1} & 0 \\
0 & 1
\end{array}\right)\right)|a|^{s-1 / 2} d^{\times} a \\
L_{v}(s, f, \chi)=\int_{k_{v}^{\times}} W_{v}\left(\left(\begin{array}{cc}
a \delta_{v}^{-1} & 0 \\
0 & 1
\end{array}\right)\right)|a|^{s-1 / 2} \chi(a) d^{\times} a .
\end{gathered}
$$

It follows that

$$
\begin{aligned}
J_{v}(2 s-1 / 2) & =\frac{1}{2}\left(L(s, f)+L_{v}(s, f, \chi)\right) \\
& =\frac{1+\eta(\varpi)|\varpi|^{2 s}}{\left(1-\alpha^{2}|\varpi|^{2 s}\right)\left(1-\beta^{2}|\varpi|^{2 s}\right)} \\
& =\frac{L_{v}\left(2 s, \operatorname{sym}^{2} f\right)}{\zeta_{k_{v}}(4 s)} .
\end{aligned}
$$

Take $s=1 / 2$. We obtain

$$
j\left(H_{v}, \sigma_{v}, \phi_{v}, \psi_{v}\right)=\frac{L\left(1, \operatorname{sym}^{2} \sigma_{v}\right)}{\zeta_{v}(2)}
$$

This is exactly the factor predicted by Theorem 1.2.1. More precisely, we have shown the following:

Lemma 5.2.1. Assume that $\phi$ is invariant under the standard maximal compact subgroups of $G\left(\mathbb{A}_{k}\right)$ and $\mathrm{SL}_{2}\left(\mathbb{A}_{k}\right)$, respectively, and is decomposable:

$$
\phi=\otimes \phi_{v} .
$$


Then the periods of $\theta_{\phi}^{f}(g)$ is also decomposable:

$$
\ell_{H, \tau}\left(\theta_{\phi}^{f}\right)=c \cdot \prod_{v} j\left(H_{v}, \sigma_{v}, \phi_{v}, \psi_{v}\right)
$$

where

- $c$ is a constant depending only on $k$ and $F$;

- $j\left(H_{v}, \sigma_{v}, \phi_{v}, \psi_{v}\right)$ depends only on the field extension $F_{v} / k_{v}$, the representation $\sigma_{v}$, the order of conductor of $\psi_{v}$, the function $\phi_{v}$, and the ramification of $H$ at $v$.

Moreover $j\left(H_{v}, \sigma_{v}, \phi_{v}, \psi_{v}\right)$ has the following properties

(1) $j\left(H_{v}, \sigma_{v}, \phi_{v}, \psi_{v}\right)$ is continuous in $\phi_{v}$, analytic in complex number $s$, if $\sigma_{v}=\pi\left(|\cdot|^{s}, \eta_{v}|\cdot|^{-s}\right)$;

(2) $j\left(H_{v}, \sigma_{v}, \phi_{v}, \psi_{v}\right)$ is a rational function of $q_{v}^{1 / 2}$ over $\mathbb{C}$ when $v$ is a finite place;

(3) for a finite place $v$, one has the following expression

$$
j\left(H_{v}, \sigma_{v}, \phi_{v}, \psi_{v}\right)=\frac{L\left(1, \operatorname{sym}^{2} \sigma_{v}\right)}{\zeta_{v}(2)}
$$

in the unramified case as defined below:

$-F_{v} / k_{v}$ is unramified;

- $\psi_{v}$ is unramified;

- $\phi_{v}$ is the characteristic function of standard lattice $V\left(\mathcal{O}_{v}\right)$;

- $H$ is split at $v$;

(4) if $\psi_{v}^{\prime}(x)=\psi_{v}\left(t^{2} x\right)$ is another character with $t \in k_{v}^{\times}$, then

$$
j\left(H_{v}, \sigma_{v}, \phi_{v}^{\prime}, \psi_{v}^{\prime}\right)=\eta(t)|t|^{2} j\left(H_{v}, \sigma_{v}, \omega_{\psi_{v}}\left(m(t)^{-1}\right) \phi_{v}^{\prime}, \psi_{v}\right) .
$$

5.3. On normalization of theta lifting. Let $\pi$ be a cuspidal representation of $G\left(\mathbb{A}_{k}\right)=\operatorname{GSO}\left(V\left(\mathbb{A}_{k}\right)\right)$ which is a lifting of a representation $\sigma$ of $G^{\prime}\left(\mathbb{A}_{k}\right)=$ $\mathrm{GL}_{2}\left(\mathbb{A}_{k}\right)_{+}$. Let $\varphi$ and $f$ be the new forms in $\pi$ and $\sigma$ respectively.

To say that $\varphi$ is a new form for $\pi$ means $\varphi$ will transform according to a character of a suitable compact subgroup $U$ of $G\left(\mathbb{A}_{k}\right)$. Any two vectors in the space of $\pi$ satisfying the same transformation rules under $U$ will be multiples of each other. For the case needed in this paper (see section 5.4 below), $U$ will be a maximal compact subgroup of $G\left(\mathbb{A}_{k}\right)$ and the new forms are spherical vectors in the space of $\pi$.

Thus if $\phi \in \mathcal{S}\left(V\left(\mathbb{A}_{k}\right)\right)$ is a Schwartz function satisfying this same transformation rule under $U$ then there is a constant $c(\sigma, \phi)$ such that

$$
\theta_{\phi}^{f}=c(\sigma, \phi) \varphi \text {. }
$$


We will show that when $\phi$ is decomposable. say $\phi=\otimes_{v} \phi_{v}$, then $c(\sigma, \phi)$ is decomposable

$$
c(\sigma, \phi)=\prod_{v} c\left(\sigma_{v}, \phi_{v}\right)
$$

with the following properties:

- for all good place $v, c\left(\sigma_{v}, \phi_{v}\right)=1$;

- for bad places $c\left(\sigma_{v}, \phi_{v}\right)$ is continuous in the variable $\phi_{v}$ and analytic in coefficients of $L\left(s, \sigma_{v}\right)$.

One natural approach to the study $c(\sigma, \phi)$ is to compute the Whittaker coefficient $W_{\phi}^{f}(g)$ of $\theta_{\phi}^{f}$ and compare it with $W_{\varphi}(g)$. At this stage, it seems hard to see such decomposability. Dually we may try to compute the Fourier coefficients of $\theta_{\phi}^{\varphi}$ which will involve the computation of periods $\ell_{H}(\varphi)$. When $H$ is split, such a period has been computed in $\S 5.1$.

Let us start with the expression

$$
c(\sigma, \phi)=\frac{\left(\theta_{\phi}^{f}, \varphi\right)_{G}}{(\varphi, \varphi)_{G}} .
$$

Here the inner product is taken on $G(k) \backslash G\left(\mathbb{A}_{k}\right)$. Recall that

$$
\theta_{\phi}^{f}(g)=\int_{G_{1}^{\prime}(k) \backslash G_{\lambda(g)}^{\prime}(\mathbb{A})} \theta_{\phi}(g, h) f(h) d h
$$

where $G_{\lambda}^{\prime}$ is the set of elements in $G^{\prime}$ with determinant $\lambda$, viewed as a homogeneous space of $G_{1}^{\prime}=\mathrm{SL}_{2}$. It follows that

$$
\begin{aligned}
\left(\theta_{\phi}^{f}, \varphi\right)_{G} & =\int_{\left(G \times G^{\prime}\right)_{\Delta}(k) \backslash\left(G \times G^{\prime}\right)_{\Delta}(\mathbb{A})} \theta_{\phi}(g, h) f(h) \bar{\varphi}(g) d(g, h) \\
& =\left(f, \widetilde{\theta}_{\phi}^{\varphi}\right)_{G^{\prime}}
\end{aligned}
$$

where subscript $\Delta$ means elements with the same determinants or similitudes, and $\widetilde{\theta}_{\phi}^{\varphi}$ is the lifting of $\varphi$ using kernel $\bar{\theta}_{\phi}$. Thus we have the expression:

$$
c(\sigma, \phi)=\frac{\left(f, \widetilde{\theta}_{\phi}^{\varphi}\right)}{(f, f)} \cdot \frac{(f, f)}{(\varphi, \varphi)} .
$$

The first term is the ratio of $\widetilde{\theta}_{\phi}^{\varphi}$ over $f$ which be computed by comparing the Fourier coefficients. More precisely, let $\widetilde{W}_{\phi}^{\varphi}$ be the Whittaker function of $\widetilde{\theta}_{\phi}^{\varphi}$ then

$$
\frac{\left(f, \widetilde{\theta}_{\phi}^{\varphi}\right)}{(f, f)}=\frac{\widetilde{W}_{\phi}^{\varphi}}{W_{f}} .
$$


The other term is essentially equal to $L\left(1, \operatorname{Sym}^{2} f\right) / L\left(1, \operatorname{Sym}^{2} \varphi\right)$.

The computation of $\widetilde{W}_{\phi}^{\varphi}$ is similar to the proof of Proposition 4.2.1. By the definition of the Weil representation, we have

$$
\begin{aligned}
& \widetilde{W}_{\phi}^{\varphi}(x)=\int_{k \backslash \mathbb{A}_{k}} \widetilde{\theta}_{\phi}^{\varphi}(n(t) h) \psi(-t) d t \\
& =\int_{G_{1}(k) \backslash G_{\operatorname{det} h}\left(\mathbb{A}_{k}\right)} \varphi(g) \sum_{\xi \in V_{-1}(k)} \overline{\omega(g, x) \phi(\xi)} d g \\
& =|\operatorname{det} x|^{-1} \int_{G_{1}(k) \backslash G_{\operatorname{det} x}\left(\mathbb{A}_{k}\right)} \varphi(g) \sum_{\xi \in V_{-1}(k)} \overline{\omega\left(x_{0}\right) \phi\left(g^{-1} \xi\right)} d g .
\end{aligned}
$$

Here $V_{-1}$ denotes the set of elements of norm -1 which is a single orbit under $G_{1}(k)$, and $x_{0}=\left(\begin{array}{c}1 \\ \operatorname{det} x^{-1}\end{array}\right) x$. Let $H$ be the stabilizer of $e:=\left(\begin{array}{c}1 \\ -1\end{array}\right) \in V_{-1}$ in $G_{1}$. Then $H$ is the subgroup corresponding to $\epsilon=1$.

$$
\begin{aligned}
\widetilde{W}_{\phi}^{\varphi}(x) & =|\operatorname{det} x|^{-1} \int_{H(k) \backslash G_{\operatorname{det} x}\left(\mathbb{A}_{k}\right)} \varphi(g) \overline{\omega\left(x_{0}\right) \phi\left(g^{-1} e\right)} d g \\
& =|\operatorname{det} x|^{-1} \int_{H\left(\mathbb{A}_{k}\right) \backslash G_{\operatorname{det} x}\left(\mathbb{A}_{k}\right)} \ell(g, \varphi) \overline{\omega\left(x_{0}\right) \phi\left(g^{-1} e\right)} d g .
\end{aligned}
$$

Here $\ell(g, \varphi)$ is the period of $r(g) \varphi$ :

$$
\ell(g, \varphi)=\int_{H(k) \backslash H\left(\mathbb{A}_{k}\right)} \varphi(h g) d h .
$$

This period can be computed using method in $\S 5.1$. To use notation in $\S 5.1$, we let $\alpha \in \mathrm{GL}_{2}(F)$ such that $\alpha H \alpha^{-1}=H_{0}:=\mathrm{GL}_{2}(k)$ and transform everything in terms of $H_{0}$. Now we copy some computations from $\S 5.1$ : 


$$
\begin{aligned}
\ell(g, \varphi) & =\int_{H_{0}(k) \backslash H_{0}\left(\mathbb{A}_{k}\right)} \varphi(h \alpha g) d h \\
& =c_{1} \operatorname{Res}_{s=1 / 2} \int_{H_{0}(k) \backslash H_{0}\left(\mathbb{A}_{k}\right)} \varphi(h \alpha g) E(h, s) d h \\
& =c_{1} \operatorname{Res}_{s=1 / 2} \int_{B(k) \backslash H_{0}\left(\mathbb{A}_{k}\right)} \varphi(h \alpha g) f(h, s) d h \\
& =c_{1} \operatorname{Res}_{s=1 / 2} \int_{N\left(\mathbb{A}_{k}\right) B(k) \backslash H_{0}\left(\mathbb{A}_{k}\right)} \int_{N(k) \backslash N\left(\mathbb{A}_{k}\right)} \varphi(n h \alpha g) d n f(h, s) d h \\
& =c_{1} \operatorname{Res}_{s=1 / 2} \int_{N\left(\mathbb{A}_{k}\right) B(k) \backslash H_{0}\left(\mathbb{A}_{k}\right)} \sum_{\xi \in k^{\times}} W_{\varphi}\left(\left(\begin{array}{ll}
\xi \xi_{0} & \\
1
\end{array}\right) h \alpha g\right) f(h, s) d h \\
& =c_{1} \operatorname{Res}_{s=1 / 2} \int_{N\left(\mathbb{A}_{k}\right) \backslash H_{0}\left(\mathbb{A}_{k}\right)} W_{\varphi}\left(\left(\begin{array}{c}
\xi_{0} \\
1
\end{array}\right) h \alpha g\right) f(h, s) d h
\end{aligned}
$$

Take $\varphi=\otimes_{v} \varphi_{v}$ and $f=\otimes_{v} f_{v}$ to be factorizable. Then by the uniqueness of local Whittaker models, we have

$$
W_{\varphi}(g)=\prod_{v} W_{v}\left(g_{v}\right)
$$

where $W_{v}(g)$ is the local Whittaker function attached to $\varphi_{v}$. It follows that the last integral is a product of the following local integrals over places of $k$ for the real part of $s$ large:

$$
\int_{N\left(k_{v}\right) \backslash H_{0}\left(k_{v}\right)} W_{\varphi_{v}}\left(\left(\begin{array}{ll}
\xi_{0} & \\
& 1
\end{array}\right) h \alpha g\right) f_{v}(h, s) d h .
$$

For places $v$ of $k$ where $\alpha_{v} g_{v} \in U_{v}$, these local integrals equal to

$$
\left|\xi_{0} \delta_{v}\right|^{1 / 4-s / 2} L_{v}\left(s+1 / 2, \operatorname{Sym}^{2} \sigma_{v}\right) \zeta_{v}(s+1 / 2) \zeta_{v}(2 s+1)^{-1} \text {. }
$$

If $\alpha_{v} g_{v} \notin U_{v}$, it can be expressed as a product of

$$
L_{v}\left(s+1 / 2, \operatorname{Sym}^{2} \sigma_{v}\right) \cdot \zeta_{v}(s+1 / 2)
$$

times a polynomial function in $q^{-s}$, since $\varphi_{v}$ is still spherical. Hence the local integrals have a meromorphic continuation to the complex plane $\mathbb{C}$ with the denominator given above. Therefore, if we define local function at each local place $v$ by

$$
\ell_{v}\left(g_{v}\right)=\frac{\zeta_{v}(2)}{\zeta_{v}(1)} \int_{N\left(k_{v}\right) \backslash H_{0}\left(k_{v}\right)} W_{v}\left(\left(\begin{array}{cc}
\xi_{0} & \\
& 1
\end{array}\right) h g\right) f(1 / 2, h) d h,
$$

then we have a factorization formula:

$$
\ell(g, \varphi)=c_{4} \prod \ell_{v}\left(\alpha g_{v}\right)
$$


where $c_{4}$ is a nonzero constant. Note that the definition of $\ell_{v}\left(g_{v}\right)$ does not depend on the choice of $\xi_{0}, \psi_{v}$, and is invariant under the center of $G\left(k_{v}\right)$.

Now we assume that $\phi$ is decomposable $\phi=\otimes \phi_{v}$. Then we have

$$
\widetilde{W}_{\phi}^{\varphi}(x)=\prod_{v} \widetilde{W}_{v}\left(x_{v}\right)
$$

with

$$
\widetilde{W}_{v}\left(x_{v}\right)=\left|\operatorname{det} x_{v}\right|^{-1} \int_{H_{0}\left(k_{v}\right) \backslash G_{\operatorname{det} x \lambda(\alpha)}\left(k_{v}\right)} \ell_{v}(g) \overline{\omega\left(x_{0, v}\right) \phi_{v}\left(g^{-1} \alpha e\right)} d g .
$$

It is easy to check that $\widetilde{W}_{v}$ here is a Whittaker function with respect to the character $\psi_{v}$. To understand the dependence on $\alpha$, we notice that for any fixed nonzero trace free element $\xi$ we may choose $\alpha$ such that

$$
\alpha e=e_{\xi}:=\left(\begin{array}{c}
\xi \\
-\xi
\end{array}\right) \text {. }
$$

Thus we have the expression

$$
\widetilde{W}_{v}\left(x_{v}\right)=\left|\operatorname{det} x_{v}\right|^{-1} \int_{H_{0}\left(k_{v}\right) \backslash G_{-\operatorname{det} x \cdot \xi^{2}}\left(k_{v}\right)} \ell_{v}(g) \overline{\omega\left(x_{0, v}\right) \phi_{v}\left(g^{-1} e_{\xi}\right)} d g .
$$

Since $\ell_{v}(g)$ is invariant if $g$ is replaced by $g t$ with $t \in k^{\times}=Z(G)$, we see that $\widetilde{W}_{v}\left(x_{v}\right)$ does not depend on $\xi$.

The dependence of $\widetilde{W}_{v}$ on $\psi_{v}$ is through the norm $\left|\delta_{v}\right|$ of its conductor. We want to show that this dependence can be extended to the class of $\delta_{v}$ modulo $\mathcal{O}_{v}^{\times} \cdot\left(k_{v}^{\times}\right)^{2}$. Let $\psi_{v}^{\prime}(x)=\psi_{v}\left(t^{2} x\right)$ be another character with a $t \in k_{v}^{\times}$. Let $\widetilde{W}^{\prime}$ be the Whittaker function of a different function $\phi_{v}^{\prime}$ and the Weil representation $\omega^{\prime}(x)=\omega\left(m(t) x m(t)^{-1}\right)$ with respect to the character $\psi_{v}^{\prime}$. Since $\ell_{v}(g)$ does not depend on the choice of $\psi_{v}$, we have

$$
\begin{aligned}
\widetilde{W}_{v}^{\prime}\left(x_{v}\right) & =\left|\operatorname{det} x_{v}\right|^{-1} \int_{H_{0}\left(k_{v}\right) \backslash G_{-\operatorname{det} x \cdot \xi^{2}}\left(k_{v}\right)} \ell_{v}(g) \overline{\omega^{\prime}\left(x_{0, v}\right) \phi_{v}^{\prime}\left(g^{-1} e_{\xi}\right)} d g \\
& =\left|\operatorname{det} x_{v}\right|^{-1} \int_{H_{0}\left(k_{v}\right) \backslash G_{-\operatorname{det} x \cdot \xi^{2}}\left(k_{v}\right)} \ell_{v}(g) \overline{\omega\left(m(t) x_{0, v} m(t)^{-1}\right) \phi_{v}^{\prime}\left(g^{-1} e_{\xi}\right)} d g .
\end{aligned}
$$

In other words, we have

$$
\widetilde{W}_{v}^{\prime}\left(x_{v}\right)=W_{v}\left(m(t) x_{v}\right)=\eta_{v}(t) W_{v}\left(\left(\begin{array}{cc}
t^{2} \\
& 1
\end{array}\right) x_{v}\right)
$$

where $\widetilde{W}$ is the Whittaker function with respect to $\psi_{v}$ and the function $\phi_{v}=$ $\omega\left(m(t)^{-1}\right) \phi_{v}^{\prime}$.

Now we want to evaluate $\widetilde{W}_{v}(1)$ at the following nice place

- $\phi_{v}$ is the characteristic function of $V\left(\mathcal{O}_{v}\right)$; 
- $F_{v} / k_{v}$ is unramified.

- $\psi_{v}$ is unramified.

In this case, we may choose $\xi_{v}$ so that $\left|\xi_{v}\right|=1$. Thus have

$$
\widetilde{W}_{v}(1)=\int_{H_{0}\left(k_{v}\right) \backslash G_{-\xi^{2}}\left(k_{v}\right)} \ell_{v}(g) \overline{\phi_{v}\left(g^{-1} e_{\xi}\right)} d g .
$$

where $G_{-\xi^{2}}\left(k_{v}\right)^{0}$ denotes the subset of elements $g$ such that $g^{-1} e$ is integral. It is not difficult to show that $G_{\xi^{2}}\left(k_{v}\right)^{0}$ has decomposition $H\left(k_{v}\right) U_{-\xi^{2}}$ where $U_{-\xi^{2}}$ is the subgroup of $G_{-\xi^{2}}\left(k_{v}\right)$ fixing lattice $V\left(\mathcal{O}_{v}\right)$. Thus

$$
\widetilde{W}_{v}(1)=\ell_{v}(1)=L\left(1, \operatorname{sym}^{2} \sigma_{v}\right) .
$$

In summary, we have shown that $\widetilde{\theta}_{\phi}^{\varphi}$ has the Whittaker function $\widetilde{W}_{\phi}^{\varphi}$ which is decomposable:

$$
\widetilde{W}_{\phi}^{\varphi}=\prod_{v} \widetilde{W}_{v}
$$

with

$$
\widetilde{W}_{v}(e)=L\left(1, \operatorname{Sym}^{2} \sigma_{v}\right)
$$

for all good places $v$ of $k$. Since $\widetilde{\theta}_{\phi}^{\varphi}$ is a multiple of $f$, by comparing their Fourier coefficients, we have that

$$
\widetilde{\theta}_{\phi}^{\varphi}=\widetilde{W}\left(\begin{array}{cc}
\delta^{-1} & 0 \\
0 & 1
\end{array}\right) \cdot f
$$

Let $c_{v}\left(\sigma_{v}, \phi_{v}, \psi_{v}\right)$ be the constant defined by

$$
c_{v}\left(\sigma_{v}, \phi_{v}, \psi_{v}\right)=L\left(1, \operatorname{Sym}^{2} \sigma_{v}\right)^{-1} \cdot \widetilde{W}_{v}\left(\begin{array}{cc}
\delta_{v}^{-1} & 0 \\
0 & 1
\end{array}\right) .
$$

Then

$$
\widetilde{\theta}_{\phi}^{\varphi}=c \cdot L\left(1, \operatorname{sym}^{2} \sigma\right) \cdot \prod c\left(\sigma_{v}, \phi_{v}, \psi_{v}\right) \cdot f
$$

where $c$ is a nonzero constant independent of $\sigma$. Plugging this to our earlier expression, we obtain

$$
c(\sigma, \phi)=c \cdot L\left(1, \operatorname{sym}^{2} \sigma\right) \cdot \prod c\left(\sigma_{v}, \phi_{v}, \psi_{v}\right) \cdot \frac{(f, f)}{(\varphi, \varphi)} .
$$

On the other hand, $\|f\|^{2}$ and $\|\varphi\|^{2}$ are $L(1, \mathrm{Ad} \pi)$ and $L(1, \mathrm{Ad} \sigma)$ respectively. By the following identity:

$$
L\left(1, \operatorname{Sym}^{2} \sigma\right) L(1, \operatorname{Ad} \sigma)=L(1, \eta \otimes \operatorname{Ad} \sigma) L(1, \operatorname{Ad} \sigma)=L(1, \operatorname{Ad} \pi),
$$

we have the following 
Lemma 5.3.1. The constant $c(\phi, \sigma)$ has a decomposition

$$
c(\sigma, \phi)=c_{0} \prod_{v} c\left(\sigma_{v}, \phi_{v}, \psi_{v}\right),
$$

where

- $c_{0}$ is a nonzero constant depending only on extension $F / k$;

- $c\left(\sigma_{v}, \phi_{v}, \psi_{v}\right)$ depends only on $\phi_{v}$ and $\sigma_{v}$, and the order of $\psi_{v}$.

Moreover $c\left(\sigma_{v}, \phi_{v}, \psi_{v}\right)$ has the following properties:

(1) $c\left(\sigma_{v}, \phi_{v}, \psi_{v}\right)$ is continuous in $\phi_{v}$ and analytic in $s \in \mathbb{C}$ if

$$
\sigma_{v}=\pi\left(|\cdot|^{s}, \eta_{v}|\cdot|^{-s}\right)
$$

(2) for $v$ a finite place, $c\left(\sigma_{v}, \phi_{v}, \psi_{v}\right)$ is a rational function of $q_{v}^{s}$ over $\mathbb{C}$;

(3) for $v$ a finite place, $c\left(\sigma_{v}, \phi_{v}, \psi_{v}\right)=1$ if the following unramified conditions hold at $v$ :

- $\psi_{v}$ is unramified,

- $\phi_{v}$ is the characteristic function of $V\left(\mathcal{O}_{v}\right)$,

- $F_{v} / k_{v}$ is unramified;

(4) if $\psi_{v}^{\prime}(x)=\psi_{v}\left(t^{2} x\right)$ is another character of $k_{v}$ with $t \in k_{v}^{\times}$, and $\phi_{v}^{\prime}$ is another function on $V\left(k_{v}\right)$, then

$$
c\left(\sigma_{v}, \phi_{v}^{\prime}, \psi_{v}^{\prime}\right)=\eta_{v}(t) c\left(\sigma_{v}, \omega\left(m(t)^{-1}\right) \phi_{v}^{\prime}, \psi_{v}\right) .
$$

5.4. On ratios. We complete here the proof of Theorem 1.2.1. We fix a BruhatSchwartz function $\phi$ which is invariant under the maximal compact subgroups of $G\left(\mathbb{A}_{k}\right)$ and $\mathrm{SL}_{2}\left(\mathbb{A}_{k}\right)$, such that $c(\phi, \sigma) \neq 0$, and is decomposable:

$$
\phi=\otimes \phi_{v} .
$$

The period of the newform $\varphi$ is given by Lemma 4.3.1:

$$
\ell(\varphi)=c(\sigma, \phi)^{-1} J(1 / 2) .
$$

We also fix an additive character $\psi$ of $k \backslash \mathbb{A}_{k}$. Our main results in the last section show that the right hand side is decomposable and agrees with Theorem 1.2.1 for good places. Let $\alpha\left(H_{v}, \sigma_{v}, \psi_{v}\right)$ be the function defined by

$$
\alpha\left(H_{v}, \sigma_{v}, \psi_{v}\right) \beta\left(H_{v}, \sigma_{v}\right)=c\left(\sigma_{v}, \phi_{v}, \psi_{v}\right)^{-1} j\left(H_{v}, \sigma_{v}, \phi_{v}, \psi_{v}\right)
$$

where $\beta\left(H_{v}, \sigma_{v}\right)$ is the local term at $v$ in the formula in Theorem 1.2.1:

$$
\beta\left(H_{v}, \sigma_{v}\right)=\frac{L\left(1, \operatorname{Sym}^{2} \sigma_{v}\right)}{\sqrt{d_{v}} \zeta_{v}(2)} \begin{cases}1 & \text { if } H_{v} \text { is split } \\ \frac{\chi_{v}\left(\varpi_{v}\right)-\chi_{v}\left(\varpi_{v}\right)^{-1}}{q_{v}^{1 / 2}-q_{v}^{-1 / 2}} . & \text { if } H_{v} \text { is not split. }\end{cases}
$$

The Lemmas proved in the last subsections imply the following: 
Lemma 5.4.1. Let $\alpha(H, \sigma)$ be the ratio of the two sides in the formula in Theorem 1.2.1. Then we have decomposition:

$$
\alpha(H, \sigma)=\alpha_{0} \prod_{v} \alpha\left(H_{v}, \sigma_{v}, \psi_{v}\right)
$$

where

- $\alpha_{0}$ is a constant depending only on $F / k$;

- $\alpha\left(H_{v}, \sigma_{v}, \psi_{v}\right)$ depends only on $\sigma_{v}, \psi_{v}$, and the ramification of $H$ at $v$;

Moreover, $\alpha\left(H_{v}, \sigma_{v}, \psi_{v}\right)$ has the following properties:

(1) $\alpha\left(H_{v}, \sigma_{v}, \psi_{v}\right)$ is analytic in $s \in \mathbb{C}$ if $\sigma_{v}=\left(|\cdot|{ }^{s}, \eta_{v}|\cdot|^{-s}\right)$;

(2) $\alpha\left(H_{v}, \sigma_{v}, \psi_{v}\right)$ is a rational function of $q_{v}^{s}$ if $v$ is a finite place;

(3) for $v$ a finite place, $\alpha\left(H_{v}, \sigma_{v}, \psi_{v}\right)=1$ if the following unramification conditions hold at $v$ :

- $\psi_{v}$ is unramified;

- $H$ is split at $v$;

- $F_{v} / k_{v}$ is unramified.

(4) if $\psi_{v}^{\prime}(x)=\psi_{v}\left(t^{2} x\right)$ another character with $t \in k_{v}^{\times}$, then

$$
\alpha\left(H_{v}, \sigma_{v}, \psi_{v}^{\prime}\right)=|t|^{-2} \alpha\left(H_{v}, \sigma_{v}, \psi_{v}\right) .
$$

We are going to show that $\alpha=1$. The idea is that Lemma 5.4.1 holds also for Eisenstein series in the sense of continuous spectrum:

$$
\alpha(H, \sigma)=\alpha_{0} \prod_{v} \alpha\left(H_{v}, \sigma_{v}, \psi_{v}\right)=1
$$

if $\sigma$ is a global principal series representation $\pi\left(|\cdot|{ }^{s}, \eta|\cdot|^{-s}\right)$.

Lemma 5.4.2. If $v$ is an infinite place then $\alpha\left(H_{v}, \sigma_{v}, \psi_{v}\right)$ is independent of $\sigma_{v}$.

Proof. In this case we may assume $k=\mathbb{Q}$. In this case, $\psi_{v}(x)=e^{ \pm 2 \pi i t^{2} x}$ for some $t \in \mathbb{R}^{\times}$. By property (4) and (1) in Lemma 5.4.1, we may assume that $t=1$ and that $s$ is imaginary. Now by property (2) of Lemma 5.4.1, for each prime $p$, $\alpha\left(H_{p}, \sigma_{p}, \psi_{p}\right)$ is a rational function of $p^{s}$ which is 1 in the unramified case. The formula (5.4.1) thus implies that $\alpha_{\infty}\left(H_{\infty}, \sigma_{\infty}, \psi_{\infty}\right)$ is a rational function of $p^{-s}$ for a set $S$ of primes $p$ ramified in $D$ or $F$. Certainly we may choose a different pair $\left(D^{\prime}, F^{\prime}\right)$ with disjoint set $S^{\prime}$ of ramified primes. So $\alpha_{\infty}\left(H_{\infty}, \sigma_{\infty}, \psi_{\infty}\right)$ have two expressions as rational functions of $p^{s}$ with $p$ 's in two disjoint sets of primes. Since $p^{s}$ for different $p$ 's are algebraically independent, all these expressions must be constants.

Lemma 5.4.3. Let $p$ be a finite prime and let $\sigma=\pi\left(|\cdot|^{s},|\cdot|^{-s} \eta\right)$ be a principal series then

$$
\alpha_{p}\left(H_{p}, \sigma_{p}, \psi_{p}\right):=\prod_{v \mid p} \alpha\left(H_{v}, \sigma_{v}, \psi_{v}\right)
$$


does not depend on $\sigma$.

Proof. We apply Lemma 5.4.1 to equation (5.4.1) to get

$$
\alpha_{0} \prod_{p} \alpha\left(H_{p}, \sigma_{p}, \psi_{p}\right)=1
$$

Now by property (2) of Lemma 5.4.1, $\alpha\left(H_{p}, \sigma_{p}, \psi_{p}\right)$ are rational functions of $p^{s}$. Since $p^{s}$ for different $p$ are algebraically independent, all $\alpha\left(H_{p}, \sigma_{p}, \psi_{p}\right)$ must be constant.

Lemma 5.4.4. For a given finite place $v$ of $k$ with residue characteristic $p$, there is a totally real quadratic extension $F^{\prime}$ of $k$, a quaternion algebra $D^{\prime}$ over $k$ split in $F^{\prime}$, and a character $\psi^{\prime}$ of $k \backslash \mathbb{A}_{k}$ such that the following conditions are satisfied:

- $F_{v}^{\prime} \simeq F_{v}$ over $v$ but $F^{\prime}$ is unramified over other places of $k$ over $p$;

- $H_{v}^{\prime} \simeq H_{v}$ but $H^{\prime}$ is split at other places of $k$ over $p$;

- $\psi_{v}^{\prime}$ has the same order as $\psi_{v}$, but $\psi^{\prime}$ is unramified at other places of $k$ over $p$.

Proof. This is obvious since each condition amounts to the existence of certain elements in $k^{\times}$satisfying some congruent conditions at the places of $k$ over $p$.

Now by Lemma 5.4.3, and property (3) of Lemma 5.4.1 we conclude that $\alpha\left(H_{v}, \sigma_{v}, \psi_{v}\right)$ is independent of $\sigma_{v}$. Thus $\alpha(H, \sigma)$ does not depend on $\sigma$. Finally, by equation (5.4.1), we conclude that $\alpha(H, \sigma)=1$.

\section{REFERENCES}

[1] Y. André G-functions and geometry. Aspects of Mathematics, vol. E13, Vieweg, Braunschweig (1989)

[2] Bloch, Spencer; Kato, Kazuya, L-functions and Tamagawa numbers of motives. The Grothendieck Festschrift, Vol. I, 333-400, Progr. Math., 86, Birkh?user Boston, Boston, MA, 1990.

[3] Clozel, Laurent; Ullmo, Emmanuel, Équidistribution de sous-variétés spécials. arXiv: math.AG/0404131 v1 6 Apr 2004.

[4] Clozel, Laurent; Ullmo, Emmanuel, Équidistribution de mesures algébriques, to appear in Compos. Math. 141 (2005), no. 5, 1255-1309.

[5] P. Deligne, Travaux de Shimura. Séminaire Bourbaki, février 1971. In: Lecture Notes in Mathematics, vol. 244, p. 123, Springer-Verlag (1971).

[6] S. Gelbart and H. Jacquet, A relation between automorphic representations of GL(2) and GL(3). Ann. Sci. École Norm. Sup. (4) 11 (1978), no. 4, 471-542.

[7] R. Godement and H. Jacquet, Zeta functions of simple algebras. Lecture Notes in Mathematics, vol. 260, Springer (1972).

[8] Harder, G.; Langlands, R. P.; Rapoport, M. Algebraische Zyklen auf Hilbert-BlumenthalFlächen. J. Reine Angew. Math. 366 (1986), 53-120.

[9] M. Harris and S. Kudla, Arithmetic automorphic forms for the nonholomorphic discrete series of $\operatorname{GSp}(2)$. Duke Math. J. 66 (1992), no. 1, 59-121. 
[10] A. Ichino, On the regularized Siegel-Weil formula. J. reine und angew Math. 539 (2001), 201-234.

[11] H. Jacquet and R. P. Langlands, Automorphic forms on GL(2). Lecture Notes in Mathematics, Vol. 114. Springer-Verlag, Berlin-New York, 1970.

[12] Jacquet, H.; Lapid, E.; Rogawski, J. Periods of automorphic forms. J. Amer. Math. Soc. 12 (1999), no. 1, 173-240.

[13] Kim, Henry H.; Shahidi, Freydoon; Cuspidality of symmetric powers with applications. Duke Math. J. 112 (2002), no. 1, 177-197.

[14] S. Kudla and S. Rallis, A regularized Siegel-Weil formula: the first term identity. Ann. of Math. 140(1994)(1), 1-80.

[15] B. J. J. Moonen, Models of Shimura varieties in mixed characteristic. Pages 267-350 in: Galois representations in arithmetic algebraic geometry, edited by A. J. Scholl and R. L. Taylor. Cambrige University Press 1998.

[16] F. Oort, Canonical lifts and dense sets of CM-points. Pages 228-234 in: Arithmetic geometry, Proc. Cortona Symposium 1994, edited by F. Catanese, Cambrige University Press (1997)

[17] S. Rallis, Langlands' functoriality and the Weil representation, Amer. J. Math. 104 (1982), 469-515.

[18] B. Roberts, The theta correspondence for similitudes. Israel J. Math. 94 (1996), 285-317.

[19] B. Roberts, Global L-packets for $\operatorname{GSp}(2)$ and theta lifts. Doc. Math. 6 (2001), 247-314 (electronic).

[20] J.-L. Waldspurger, Dmonstration d'une conjecture de dualit de Howe dans le cas p-adique, $p \neq 2$, Festschrift in honor of I. I. Piatetski-Shapiro on the occasion of his sixtieth birthday, Part I (Ramat Aviv, 1989), 267-324, Israel Math. Conf. Proc., 2, Weizmann, Jerusalem, 1990.

[21] S. Zhang, Equidistribution of CM-points on quaternon Shimura curves, Preprint.

Di-Hua Jiang

School of Mathematics

University of Minnesota Minneapolis

E-mail: dhjiang@math.umn.edu

Jian-Shu Li

Department of Mathematics

HKUST

Hong Kong

E-mail: matom@ust.hk

Shou-Wu Zhang

Department of Mathematics

Columbia University New York

E-main: szhang@math.columbia.edu 